\title{
A PAPER-BASED ELISA DEVICE FOR THE RAPID DETECTION OF ISCHEMIC STROKE
}

\author{
A Thesis \\ presented to \\ the Faculty of California Polytechnic State University, \\ San Luis Obispo
}

\author{
In Partial Fulfillment \\ of the Requirements for the Degree \\ Master of Science in Biomedical Engineering
}

by

Andrea Elisabeth Larsen

June 2019 
(C) 2019

Andrea Elisabeth Larsen

ALL RIGHTS RESERVED 
COMMITTEE MEMBERSHIP

TITLE: A Paper-Based ELISA Device for the Rapid Detection of Ischemic Stroke

AUTHOR: Andrea Elisabeth Larsen

DATE SUBMITTED: June 2019

COMMITTEE CHAIR: Dr. David Clague

Professor, Biomedical Engineering

California Polytechnic State University

COMMITTEE MEMBER: Dr. Amy Howes

Lecturer, Biological Sciences

California Polytechnic State University

COMMITTEE MEMBER: Dr. Lily Laiho

Professor, Biomedical Engineering

California Polytechnic State University 


\begin{abstract}
A Paper-Based ELISA Device for the Rapid Detection of Ischemic Stroke Andrea Elisabeth Larsen
\end{abstract}

Diagnosing an ischemic stroke accurately and quickly is essential for appropriate treatment and results in more effective management of the stroke, leading to better patient outcomes. Specifically, the determination of ischemic versus hemorrhagic stroke leads to critically different treatment paths, which would be potentially fatal if administered incorrectly. Currently, ischemic strokes are diagnosed through a series of tests, including a physical examination, CT scans, and a panel of blood tests to exclude hemorrhagic stroke or other similarly presenting conditions, in order to administer tPA (the main treatment) within the three hour window.

The discovery of biomarkers, molecules which are upregulated during the onset of a specific condition or disease, can be used to diagnose patients when integrated with an appropriate platform. The objective of this thesis was to further develop a paper-based microfluidic device for the rapid detection of ischemic stroke using biomarkers that can be detected in the blood. This device uses HRP-based enzyme linked immunosorbent assay (ELISA) technology on a cellulose paper surface to yield a sensitive (11.8 pM) assay targeting S100B, a protein released by glial cells in the brain during stroke. Wax printing allows for creation of precise hydrophilic pathways in which sample fluid can travel within the $3 \mathrm{D}$ microfluidic device to react with a variety of proteins and reagents.

This technology has the potential to be implemented globally as a point-of-care device for use in developing countries without consistent or reliable access to advanced diagnostic technology. The device is cheap to produce, portable, and results can be determined quickly and qualitatively analyzed using cell phone images, making it an accessible technology for patients around the globe. 


\section{ACKNOWLEDGMENTS}

It has been an amazing experience to complete both my undergraduate and graduate degrees at Cal Poly and I would not have enjoyed my time here as much without the continual support from those around me.

I would first like to thank my thesis advisor, Dr. David Clague, for guiding me throughout the past three years toward a focus in diagnostic medicine with both his courses and our work together on this thesis. I am grateful that he took me on as his graduate student and let me continue this project which is so important to him. Dr. Clague was continually there for me, offering support, feedback, and hours of great conversation. Without him, I would not have gotten to this point in realizing both my passions educationally and

I also want to thank my thesis committee. Thank you to Dr. Amy Howes for giving me support with the development of my thesis and to Dr. Lily Laiho for guiding me with my degree during my time at Cal Poly.

Additionally, I am very grateful for the Baker/Koob Endowment committee for funding this project so that I would be able to completely fulfill my goals. Without that essential support, this project would not have been possible.

Finally, I would like to thank my friends and family for their never ending encouragement. To my parents and sister, thank you for always being there for me, even from far away. To my friends who kept me positive and motivated throughout this process and for all of the good times spent in the ATL. I am so thankful for all of the memorable experiences we have had over the past five years and for the never ending encouragement and friendship. 


\section{TABLE OF CONTENTS}

Page

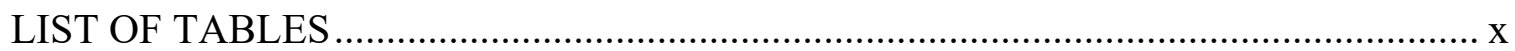

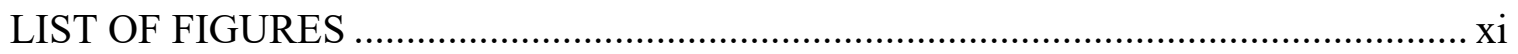

\section{CHAPTER}

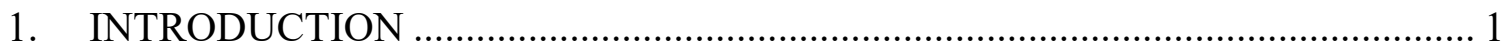

$1.1 \quad$ Specific Goals of this Thesis Project and Report................................................. 1

$1.2 \quad$ Molecular Diagnostics Technology ............................................................ 2

$1.3 \quad$ Enzyme Linked Immunosorbent Assay (ELISA) ……............................... 3

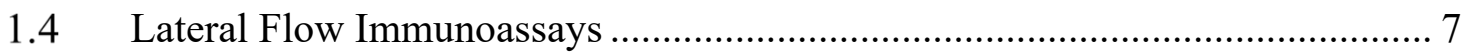

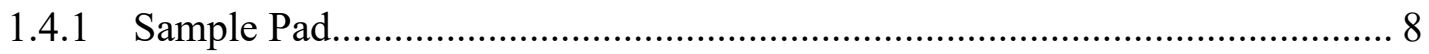

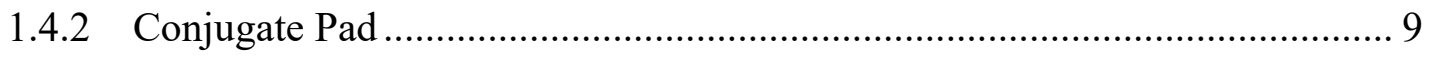

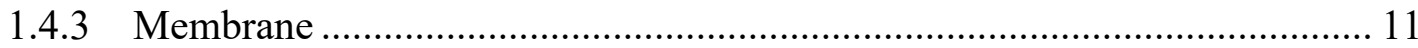

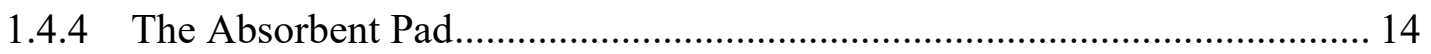

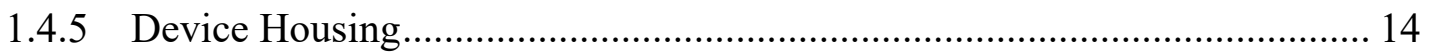

$1.5 \quad$ Paper-Based Microfluidic Assays ............................................................... 15

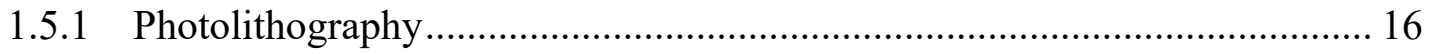

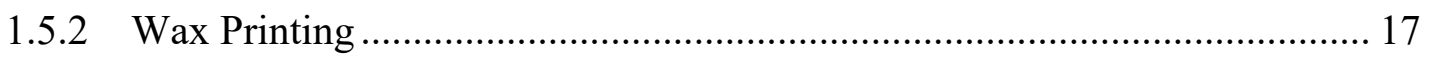

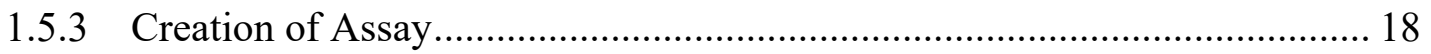

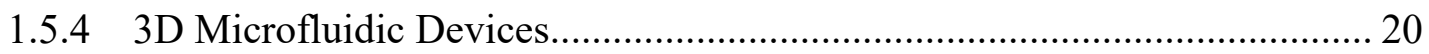

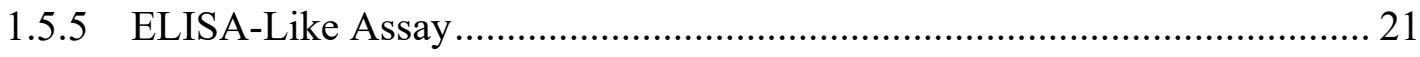

1.6 On Board Reagent Incorporation ............................................................... 23

1.7 Characteristics of Fluid Flow in Paper Microfluidics..................................... 25

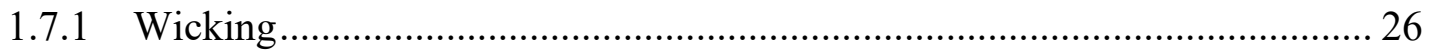

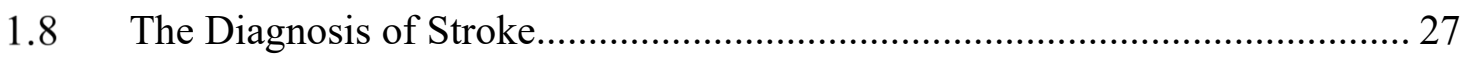




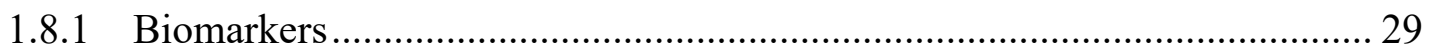

1.9 A Global Approach to Diagnostic Medicine ……………………………..... 31

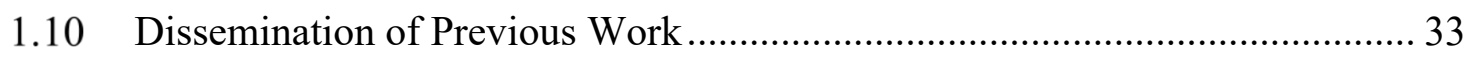

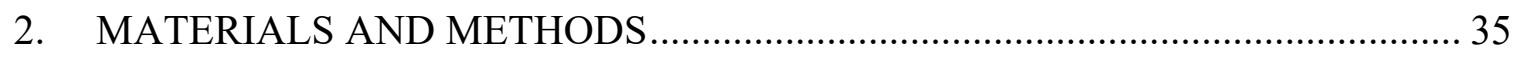

2.1 3D Paper Microfluidic Chip Fabrication ...................................................... 35

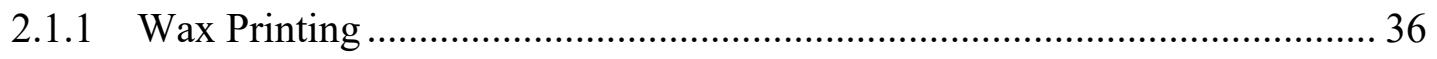

2.1.2 Housing Fabrication............................................................................. 37

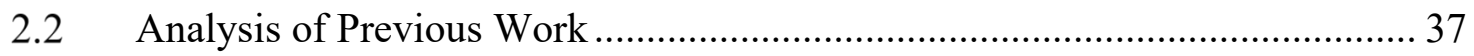

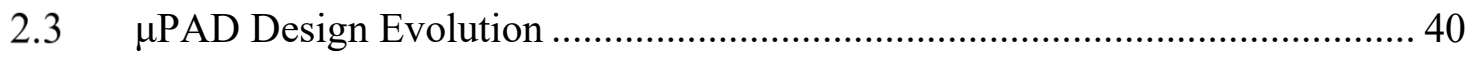

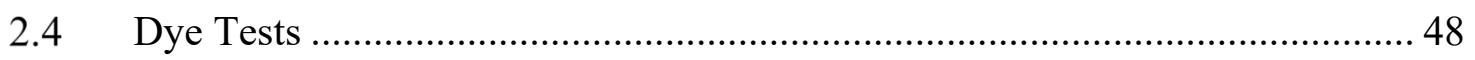

2.4.1 Single Sample Device - Taped Assembly....................................................... 48

2.4.2 Single Sample Device - Housing Assembly .................................................. 50

2.4.3 Rotating Assembly ……………………….......................................... 52

$2.5 \quad$ Housing Development …………………………..................................... 53

2.6 Enzyme Assay Development ........................................................................ 54

2.6.1 Assay Preparation Procedure …………………………………………..... 54

2.6.2 Assay Implementation Procedure …………………………................... 55

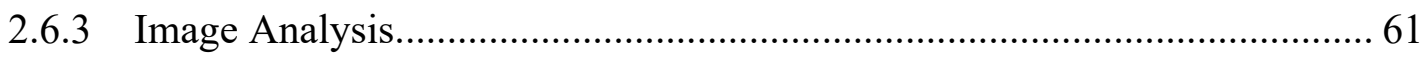

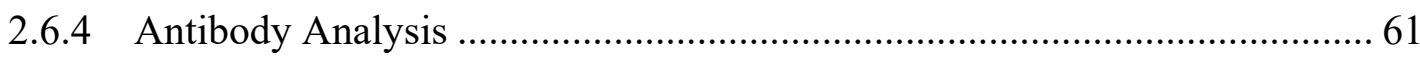

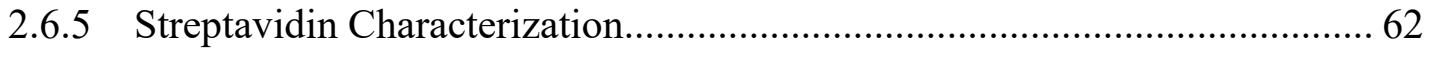

2.6.6 Optimization of Detection Antibody ......................................................... 62

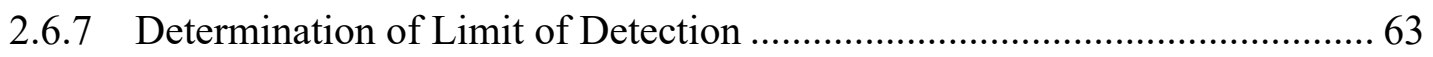

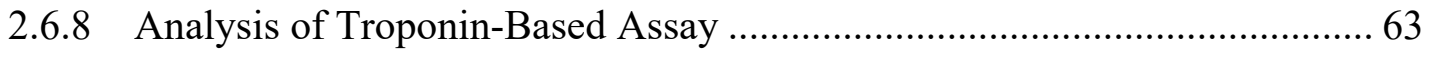

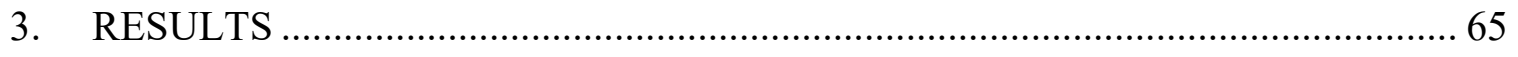




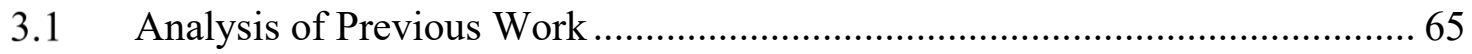

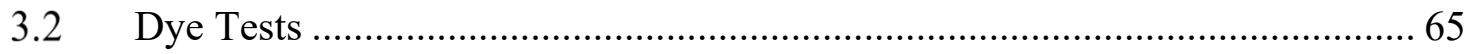

3.2.1 Single Sample Device - Taped Assembly....................................................... 65

3.2.2 Single Sample Device - Housing Assembly …………………………........... 67

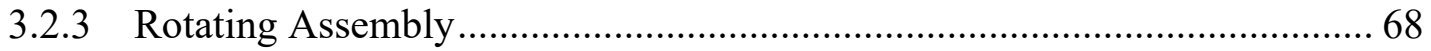

3.3 Enzyme Assay Development …………………........................................ 70

3.3.1 Antibody Reactivity Analysis ................................................................... 70

3.3.2 Streptavidin Characterization.................................................................... 71

3.3.3 Optimization of Detection Antibody ........................................................... 72

3.3.4 Determination of Limit of Detection ......................................................... 75

3.3.5 Analysis of Troponin-Based Assay ............................................................ 78

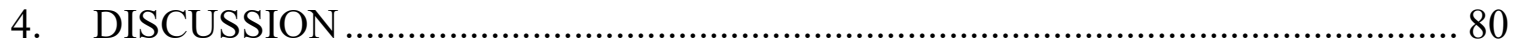

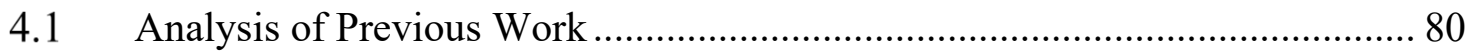

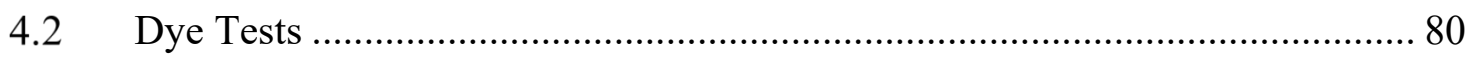

4.2.1 Single Sample Device - Taped Assembly..................................................... 81

4.2.2 Single Sample Device - Housing Assembly ................................................. 82

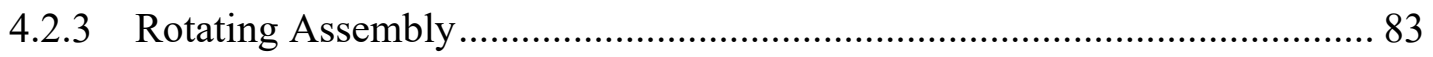

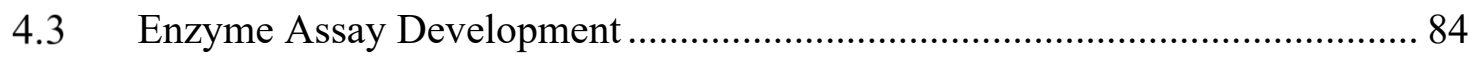

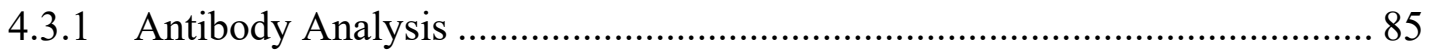

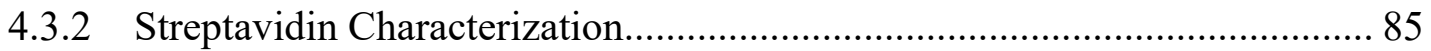

4.3.3 Optimization of Detection Antibody ………………................................ 86

4.3.4 Determination of Limit of Detection ........................................................ 89

4.3.5 Analysis of Troponin-Based Assay ............................................................. 92

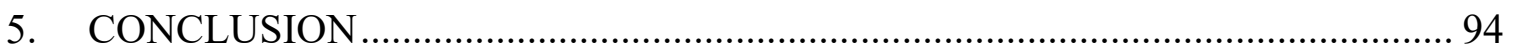




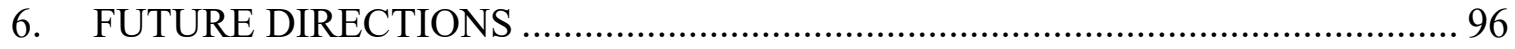

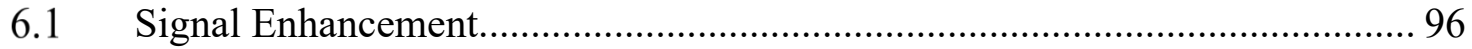

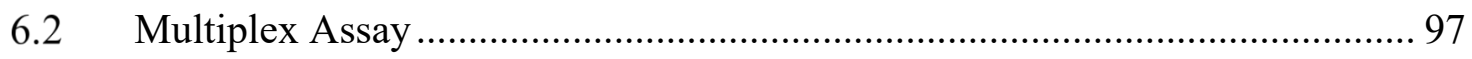

$6.3 \quad$ Long-Term Reagent Storage

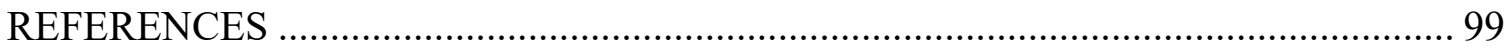

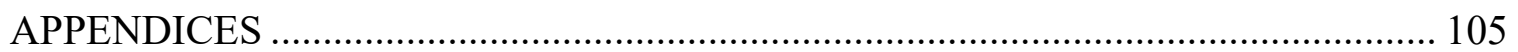

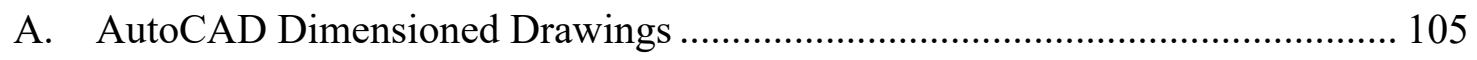

B. Enzyme Assay Development .................................................................... 110 


\section{LIST OF TABLES}

Table

Page

I. Biomarker Panels for the Diagnosis of Ischemic Stroke [46] ................................. 30

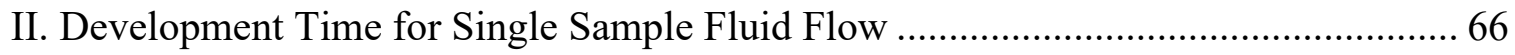

III. Development Times for Single Sample Device with Housing ................................ 68

IV. Average Development Times for Single Sample Device with Housing ................... 68

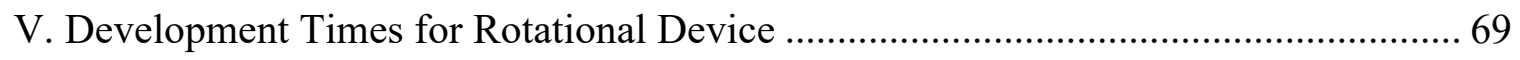

VI. Average Development Times for Rotational Device............................................ 70

VII. Color Intensity of Initial Reactivity Tests ..................................................... 71

VIII. ImageJ Analysis of Streptavidin Assay ................................................................ 72

IX. Time for Development of Detection Antibody Optimization Trials ........................ 73

X. Average Development Times for Detection Antibody Optimization Trials............... 73

XI. Development Times for Dose Response Device Trials ......................................... 76

XII. Average Development Times for Dose Response Trials ..................................... 76

XIII. ImageJ Analysis Results for the Troponin-Enabled Assay................................. 79

XIV. High Concentration Dose Response - Trial 1 ................................................ 110

XV. High Concentration Dose Response - Trial 2 ................................................ 111

XVI. High Concentration Dose Response - Trial 3 ............................................... 111

XVII. Low Concentration Dose Response - Trial 1 ............................................... 111

XVIII. Low Concentration Dose Response - Trial 2 ............................................... 112

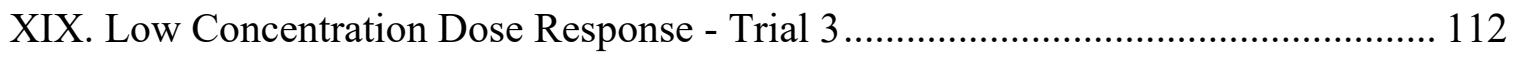

XX. Average of All Trials - Uncorrected ............................................................. 112

XXI. Average of All Trials - Corrected ............................................................ 113 


\section{LIST OF FIGURES}

Figure

Page

1. Four commonly used formats for an ELISA [6] ................................................ 4

2. Two types of sandwich ELISA, (a) direct sandwich and (b) double sandwich, adapted

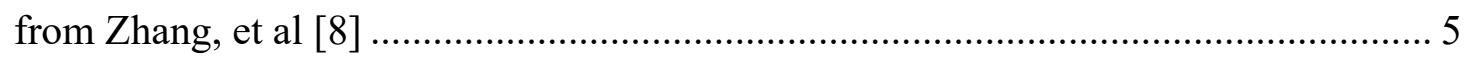

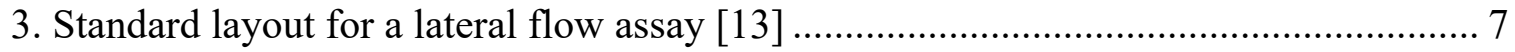

4. Test and control line antibody loading and colorimetric display [14] ...................... 12

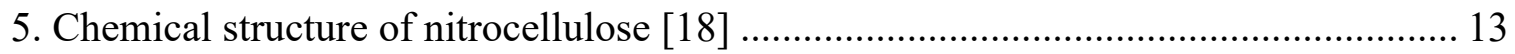

6. Hydrophilic channel created by hydrophobic barrier [22] ..................................... 16

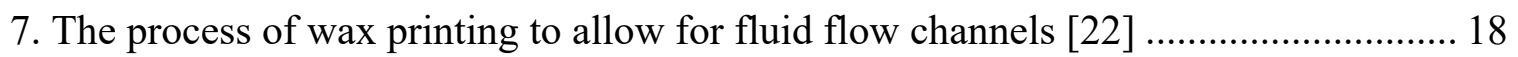

8. Progression of analytical testing of the paper microfluidic device [23] ..................... 19

9. Paper-Based ELISA Format with Photoresist on Paper [30] ................................... 21

10. Sliding Format for a P-ELISA Showing the Layers and Reagents [32] ................... 22

11. Chart showing the effect of long-term dry storage of the conjugate on assay

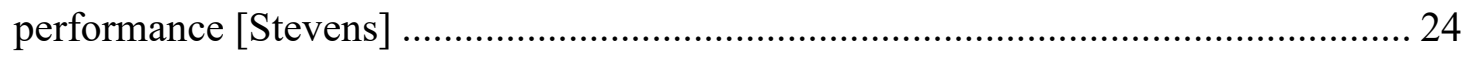

12. Previous final chip design from Holler (2016) [56].............................................. 38

13. Previous final housing from Holler (2016). A) AutoCAD design, top of housing on

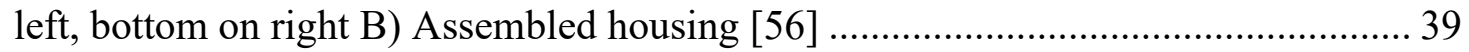

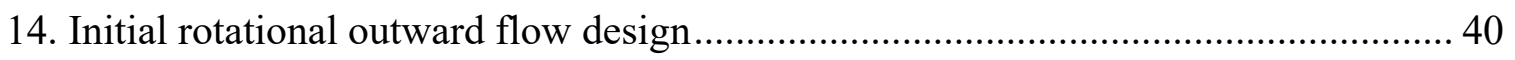

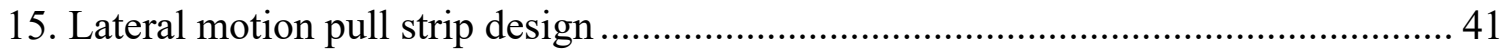

16. Outward flow design with four channels and a control pad .................................. 43

17. Outward flow design with three detection pad channels and three control pad channels. 
18. Final design for one sample fluid flow validation test........................................... 45

19. AutoCAD design and acrylic production of the one sample test flow housing.......... 46

20. Nitrocellulose star detection layer design ................................................................. 47

21. The final design for the paper-based $\mu \mathrm{PAD}$.......................................................... 48

22. Process of taping device layers together to run validation tests, in order A-H........... 50

23. Process of assembling the device layers in the housing for the one sample fluid flow

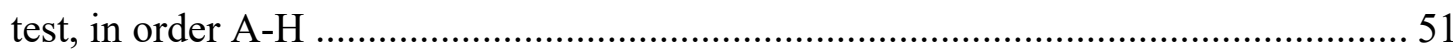

24. Nitrocellulose star design wax printed on cellulose and assembled, in order A-G .... 52

25. Housing assembly with knurled-head screws ............................................................ 53

26. Housing with set screws, A) before device assembly and B) after device assembly . 54

27. Hole-punched glass fiber for use as conjugate pads ................................................ 56

28. Glass fiber pads blocked with BSA …………………………............................ 56

29. Nitrocellulose pad with specified holes cut out .......................................................... 57

30. The process of attaching conjugate pads to nitrocellulose with double-sided tape, A) with tape backing attached and B) after tape backing is removed..............................5 58

31. Front (A) and back (B) of layer one taped to the detection nitrocellulose pad........... 58

32. Fully assembled assay device prior to sample loading ............................................. 59

33. Device after first rotation and first PBS application ................................................. 60

34. Device after second rotation and second PBS application......................................... 60

35. Fluid flow analysis of Holler's design with red dye................................................ 65

36. Progression of red dye through single sample device, A) at detection pad and B) at

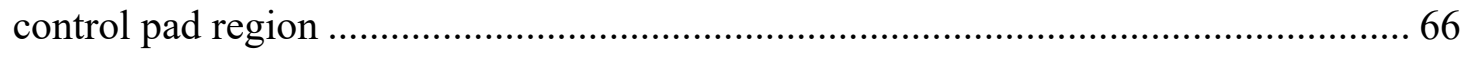

37. Flow of red dye through single sample device with housing, A-C as time elapses.... 67 
38. Results of fluid flow testing of single sample device; A) top of layer one and B) bottom of layer one showing the detection pad

39. Results of rotational design for characterization of fluid flow, A) assembled and B) disassembled 69

40. Colorimetric result of antibody analysis test ................................................ 70

41. Device used to characterize mutant vs native streptavidin .................................. 71

42. Colorimetric result for trial one of optimization of detection antibody tests.............. 72

43. All trials of signal quality in dependence of detection antibody concentration.......... 74

44. Signal quality in dependence of detection antibody concentration ........................... 74

45. Assay response to different analyte concentrations, A) high set of concentrations and

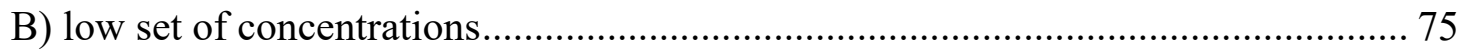

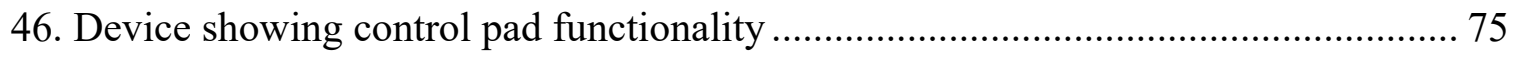

47. Dose response for different analyte concentrations fit to a $4 \mathrm{PL}$ regression curve...... 77

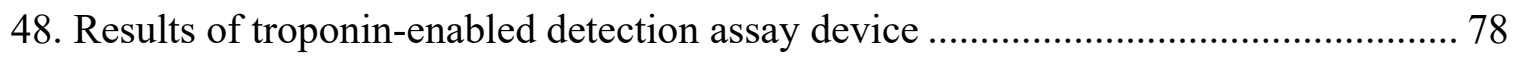

49. Single sample fluid flow design with A) flow barrier area marked in red on AutoCAD design and B) results from an incomplete trial ................................................ 81

50. Dimensioned drawing of Holler (2016) device design ........................................ 105

51. Dimensioned drawing of Holler (2016) housing design ..................................... 105

52. Dimensioned initial rotational outward flow design.......................................... 106

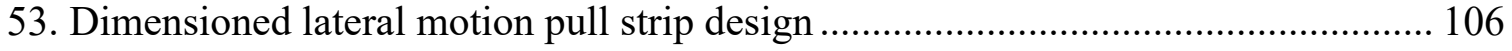

54. Dimensioned outward flow design with four channels and a control pad ............... 107

55. Dimensioned outward flow design with three detection pad channels and three control pad channels 
56. Dimensions of final design for one sample fluid flow validation test .................... 108

57. Dimensions of single sample device housing ................................................. 108

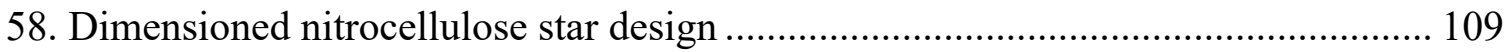

59. Dimensioned final design for the paper-based $\mu$ PAD ........................................ 109

60. Dimensioned final design for the paper-based $\mu \mathrm{PAD}$ housing ............................ 110

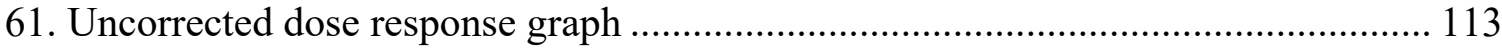

62. Dose response of device for average intensity................................................ 114 


\section{INTRODUCTION}

\subsection{Specific Goals of this Thesis Project and Report}

The purpose of this thesis project and associated report is to enable a paper-based approach to an enzyme-linked immunosorbent assay (ELISA). The main objective is to fully integrate reagents in the device to minimize the time and effort required by the user. This assay will be loaded with reagents that are able to bind to and detect the presence of biomarkers in a patient sample in order to diagnose a specific pathology. Onboard reagents, e.g. reagents that are included on the assay as opposed to added from liquid by the user, will allow for overall simplification of the test device and potential for use in developing countries due to the lower cost and rugged design. At the project's completion, we will have shown that an ELISA-like assay can be run in a paper-based format with onboard reagents for specific biomarker detection. We will accomplish this goal by:

- Design a full multi-layer paper-chip for an ELISA-like assay

- Design a housing component to apply the appropriate pressure to mediate fluid transfer between layers

- Design and apply onboard conjugate reagent

- Assemble device in housing and test fluid connectivity via dye tests

- Redesign chip and housing component and optimize fluid connectivity via dye tests

- Demonstrate ELISA-like assay on chip using antibodies and antigens

- Quantify the signal to demonstrate a standard ladder using smart phone image analysis 


\subsection{Molecular Diagnostics Technology}

Molecular diagnostics is broadly defined as the study of the specific biological markers within a patient's genome and/or proteome in order to derive a conclusion about patient health. These tests can yield diagnostic information about a patient's genetic makeup, gene expression, potential or current disease states, and genetic mutation detection. Originally, these techniques were used in a research laboratory setting and have recently begun to make their way into the clinical diagnostics realm due to high accuracy, repeatability, ease of use, and low-cost manufacturing potential all while quickly providing information about the patient's diagnosis. This diagnosis can lead to quick treatments and preventative care, improving patient outcomes and overall quality of care.

Molecular diagnostics includes experimental analyses from a variety of techniques, including polymerase chain reaction (PCR), microarray chips, and biological assays [1]. PCR uses the mechanisms behind DNA synthesis in order to target and amplify specific pathogenic DNA sequences resulting in millions of identical DNA copies [2]. This technique can be used to detect viruses, bacteria, and specific genetic sequences in the patient's genome in order to determine if the patient is suffering from a viral or bacterial infection or accurately diagnose many genetic diseases [2]. As a downside, PCR requires a skilled user and investment in high-cost equipment. Microarrays invoke a similar property of nucleotide bonding. A patient's mRNA is isolated from a sample, converted to cDNA, and labeled with a fluorescent probe. DNA is bound to the bottom of the microarray plate and the patient's sample is incubated over it, allowing the patient's cDNA to bind to the fixed DNA. Fluorescent dye intensity indicates the quantity of cDNA bound to that corresponding DNA sequence of interest. However, the bioinformatics/statistical analysis 
workload generated by high-density microarrays is very taxing [3]. The technologies within the assay category are often looking at the proteins expressed by the patient's genetic code, rather than the genetic code itself. Assays are currently developed in several forms to target specific biomarkers, which are any type of substance whose presence indicates a disease, infection, or other type of physiological characteristic [4]. Assays function on the basis of antigen-antibody bonding characteristics wherein a biomarker (antigen) can be detected using the specific and unique antibody to which it binds. These assays can be costly to develop because the antigen-antibody pair must be experimentally determined but they follow a similar design across different diagnoses.

\subsection{Enzyme Linked Immunosorbent Assay (ELISA)}

The Enzyme Linked Immunosorbent Assay (ELISA) was developed in the 1970s to target and detect specific analytes in a sample, including antibodies, antigens, proteins and glycoproteins [5]. Typically, ELISAs are run in the lab on 96-well plates due to the fact that the test method requires several wash steps, which must be run in a microplate washer and further analyzed on a microplate reader. There are several different types of ELISAs including direct, indirect, sandwich, and competitive ELISAs, which refer to the orientation of the antigen-antibody binding that occurs and which portion of the reaction is immobilized on the plate. Each of these reactions can be seen in Figure 1 below, which illustrates the difference of binding order between the types of ELISA. 


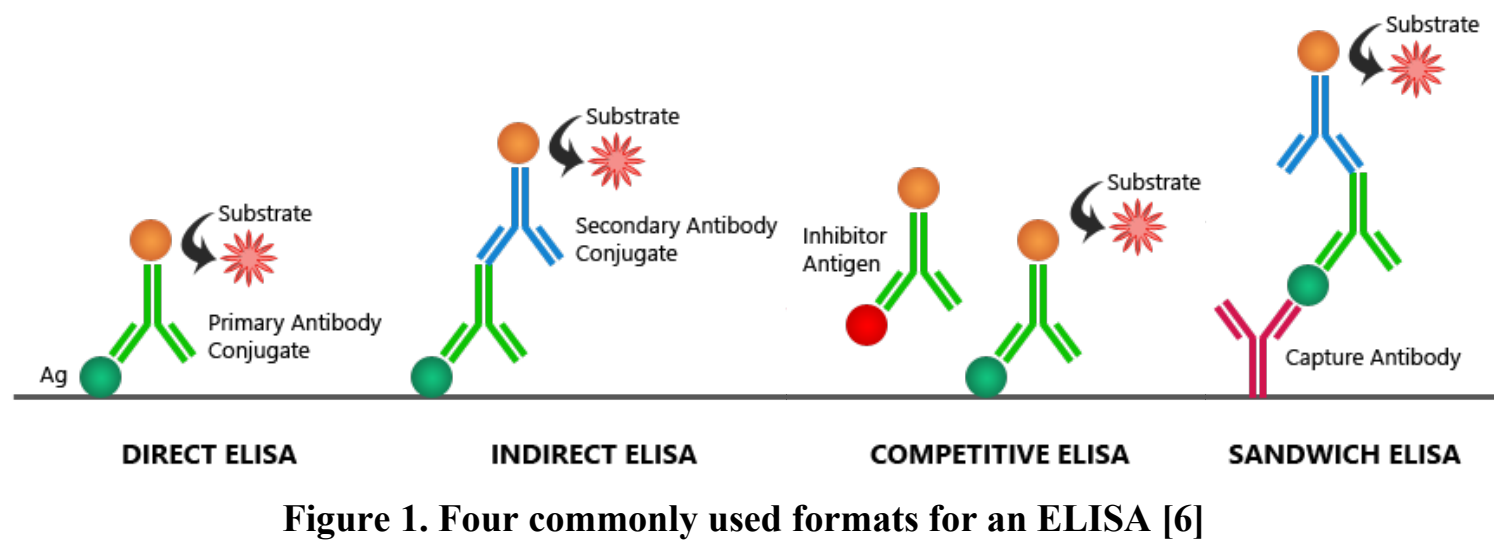

The antigen is the protein of interested and is designated with a green circle. The orange circle is a reactive enzyme which is used to cause a fluorescent or chromogenic reaction, directly indicating a positive binding result. The substrate is a red star which is what the enzyme reacts with in order to produce said fluorescence or color change. Direct and indirect ELISAs both begin with the antigen immobilized with direct adsorption to the bottom of the plate and then is detected using either one or two antibodies. Direct detection is the least accurate of the four types of ELISA but is quite common for immunohistochemical staining of tissues and cells [7]. The indirect ELISA is more specific than the direct ELISA because the secondary antibody conjugate provides another level of specificity to the primary conjugate antibody to ensure accuracy. Competitive ELISA is commonly used when the antigen is small and has only one epitope [7]. This type of ELISA involves the unlabeled antigens from the sample competing with labeled antigens (the red "inhibitor antigen," above) that are added to the plate to bind to the capture antibody. In this case, the presence of the antigen in the sample lowers the intensity of the color signal because the labeled antigens will have been forced to unbind and then subsequently washed out. 
Sandwich ELISAs are the most commonly used ELISAs to detect an antigen in solution because it is highly specific, very sensitive, and is able to detect an antigen without pretreatment of a complex sample. When referring to diagnostic tests, sensitivity is the ability of the test to correctly report positive results, whereas specificity is the ability of the test to correctly report negative results. In this assay, the antigen of interest is sandwiched between two antibodies - a capture and a detection antibody. The capture antibody is first incubated onto the plate and then the sample is washed over it. Here, the antigens bind, and the remainder of the sample is washed out. Then, the plate is incubated with a second enzyme-linked detection antibody that binds directly to the antigen, creating a "sandwich" of antibodies. Alternatively, the antigen will be bound with an unlabeled conjugate antibody which will then itself be detected with another enzyme-linked conjugate antibody. In either instance, the labeled antibody has a substrate that will change color, indicating the antigen presence [7]. Both types of sandwich ELISA can be seen in Figure 2 below.

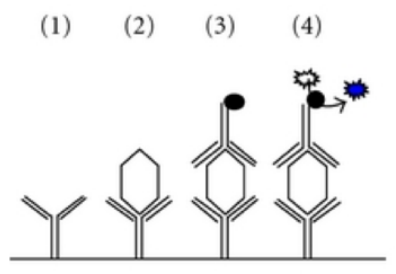

(a)

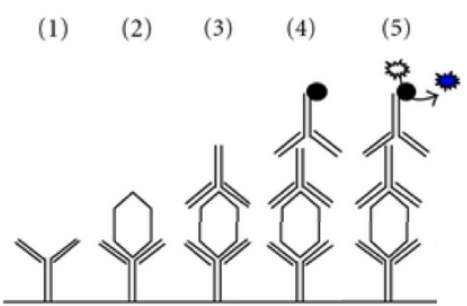

(b)

Figure 2. Two types of sandwich ELISA, (a) direct sandwich and (b) double sandwich, adapted from Zhang, et al [8]

Monoclonal antibodies are antibodies which all have a paratope against one epitope on an antigen. In contrast, polyclonal antibodies are a set of antibodies which have paratopes to a variety of epitopes on one antigen. Since ELISAs often use monoclonal antibodies, antibodies that bind to a single antigen epitope, they exhibit high specificity for 
that antigen, resulting in highly accurate tests. This accuracy is only increased with binding of additional antibodies within the ELISA because the final result will only indicate the reactions which bound every antibody successfully. For example, in the direct ELISA, only one antibody needs to bind one antigen to yield a positive result, whereas in the double sandwich ELISA, three antibodies needed to bind correctly to show a positive result, reducing the chance for a false positive. This higher complexity ELISA is therefore used due to the high sensitivity (low number of false negatives) and specificity (low number of false positives) [8]. However, even with high specificity, false negatives and positives can still occur within these tests. For ELISAs, reasons for false positives include:

1) Non-specific reaction caused by the secondary antibody

2) Hydrophobic binding of immunoglobulin components in sample to plastic surfaces

3) Ionic interaction between immunoglobulin in sample specimens and antigen

4) Immune-recognition of blocking agents by antibodies in serum specimens [10]. All of these would return a positive result even if the target antigen was not present in the sample. Further, false negative reactions can occur from:

1) The competitive inhibition of test antibodies by relevant antibodies in animal serum

2) Denaturation of enzymes conjugated to detection antibodies [10].

These errors would show a negative result despite the target antigen actually being present in the sample. Both types of incorrect results would result in misinformed patient diagnosis and potentially misdirect further treatment. These test failures can be mitigated against using BSA blocking, extensive antibody screening, and reduction or quantification of background noise [11]. 


\subsection{Lateral Flow Immunoassays}

Lateral flow immunoassays (LFA) are currently found in a variety of applications, including pregnancy tests, urine glucose tests, or tests for diseases like malaria, dengue, and typhoid fever [12]. An LFA is usually composed of the following elements: sample pad, conjugate release pad, membrane with immobilized antibodies and adsorbent pad. The components of the strip are usually fixed to an inert backing material, as seen in Figure 3 below.

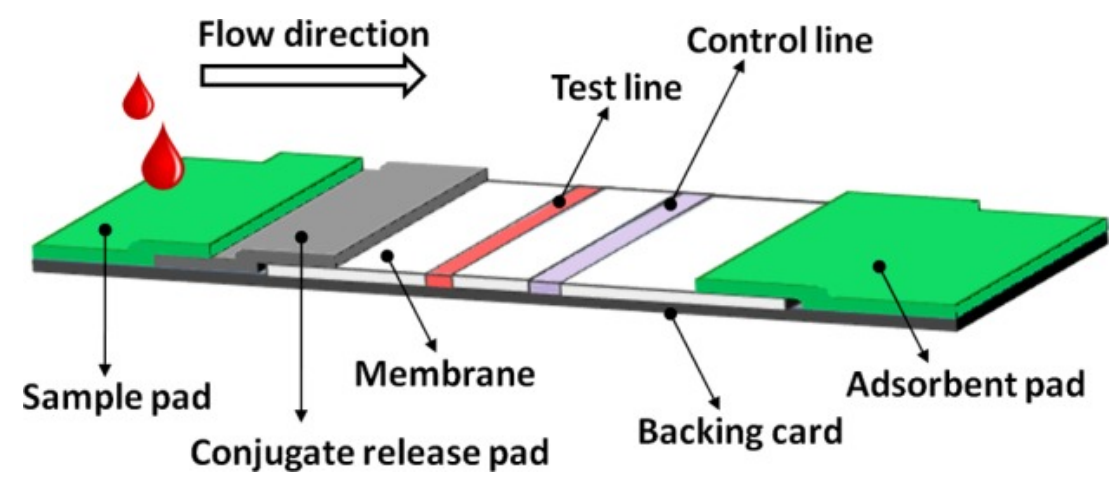

Figure 3. Standard layout for a lateral flow assay [13]

Typical materials include cast membranes (e.g., nitrocellulose, polyethersulfone), fibrous filters (e.g., cellulose, glass fiber, rayon, polyester), filters manufactured from fused particles (e.g., polyethylene), and even some woven polymers [14]. The patient sample is spotted onto the assay sample pad and then runs along the strip, leading it across the conjugate pad where the sample solubilizes the detector reagent with labeled antibodies. These antibodies bind to the biomarkers in the sample and later bind to capture antibodies on the test line to display the results of the assay. 


\subsubsection{Sample Pad}

The first portion that the sample comes into contact with is the sample pad, which is primarily used to collect the entirety of the sample and distribute it across the rest of the assay strip at a controlled rate. Lateral flow assays are capable of handling a variety of patient samples, including saliva, urine, blood plasma, et. al. These samples all have different biological and chemical makeups, from the matrix of constitutive proteins to the $\mathrm{pH}$, charge, and viscosity. These properties vary between sample type as well as between patients, depending on time of day, diet, age, etc [15]. The physical characteristics of a sample allow or prohibit the sample to flow through the entirety of the assay, therefore, an optimum set of characteristics exist that make the sample compatible with the assay. These parameters vary from assay to assay and can be achieved through a pretreatment process. Pretreatment can either occur separately from the assay - e.g. in a centrifuge to separate blood components - or on the sample pad itself. Sample treatments can include the filtering out of particulates or red blood cells, changing the $\mathrm{pH}$ of the sample, and/or actively binding sample components that can interfere with the assay in order to increase the overall sensitivity of the assay, depending on the sample type and the design of the device [14]. The treatments can include blocking reagents, proteins, detergents, and surfactants which all work toward "normalizing" the sample in the sample pad. Typically, the liquid treatment reagents and/or buffers are added onto the sample pad and allowed to dry down.

The material options for the sample pad allow for variability in sample volume, efficacy of pretreatment, and overall control of the sample. Two of the main characteristics of the material that must be considered are the tensile strength and the volume capacity (bed volume). Both of these must be optimized to yield a sample pad which is strong 
enough to undergo handling and reagent loading and has a large enough bed volume to be able to hold the entire sample. The bed volume, defined as the volume of air in the pores per unit surface area (e.g., $\mathrm{mL} / \mathrm{cm}^{2}$ ), determines the total liquid volume that can be held by the material when the pores are completely saturated [14]. Tensile strength, defined as the force at which a membrane breaks, decreases proportionately with increasing pore size, creating an optimal range for high tensile strength and large pore size [16]. Typical tensile strengths for lateral flow membranes range from 1.75 to 8.8 Newtons/cm [16]. Most commonly, cellulose fiber filters and woven meshes are used in the sample pad. Cellulose fiber pads tend to have low tensile strength and higher bed volumes, while, conversely, woven meshes tend to have low bed volumes and high tensile strength. Due to the higher bed volume, cellulose pads are often ideal because of the ability to hold any pre-treatment reagents. However, the low tensile strength relative to the woven mesh means that the cellulose pads are brittle and must be handled delicately.

\subsubsection{Conjugate Pad}

After passing through the sample pad and undergoing any necessary pretreatment, the sample flows through the conjugate pad. Aptly named, the conjugate pad contains the conjugate antibody for the biomarker which the assay is designed to detect, e.g. the antibody and antigen, respectively. Similar to the sample pad pretreatment reagents, the conjugate is dried down onto the pad and re-solubilized by the sample. The conjugate pad must be able to store the conjugate in a stable state and then release it efficiently once the sample comes into contact with the pad. In order to properly dry the conjugate to the pad, the buffer solution must contain sugars for long-term stability and ease of re-solubilization 
upon the arrival of the sample [15]. The sugar molecules in the buffer create a layer around the conjugate that works to stabilize the physical structure for long-term storage [16]. These sugar molecules dissolve in the sample fluid, allowing the conjugate to release and be carried by the fluid stream, binding to the specific antibody [16]. The conjugate is tagged with a label that will later be used to indicate capture and indicate the presence of the analyte of interest. If the conjugate and label complex is not appropriately dispensed or dried down onto the conjugate pad, then the release of the conjugate will be affected, which could have an adverse effect on the results of the test and the overall sensitivity of the assay [17]. Labels used in lateral flow assays include gold nanoparticles, colored latex beads, magnetic particles, carbon nanoparticles, selenium nanoparticles, silver nanoparticles, quantum dots, organic fluorophores, textile dyes, enzymes, liposomes and others [17]. The choice in label depends on the quantity of steps and the final detection method desired. Some of the labels listed above generate a direct signal, e.g. the gold nanoparticles showing as a deep red, while others require an additional step, e.g. the enzymes which fluoresce when an appropriate reaction substrate is added. Additionally, some labels are limited in only being qualitative in nature - only returning a yes/no response to the presence of the analyte.

The choice of material for the conjugate pad is important due to the fact that if the conjugate is not correctly bound to the pad and released with the sample, then the test will not be accurate. Since the conjugate pad needs to be able to hold a large volume, porous, non-woven filters are commonly used, which are fibers of cellulose, glass, or plastic (such as polyester, polypropylene, or polyethylene) compressed into thin pads [14]. These filters have large bed volumes and will hold the full volume of reagent require to suspend the 
labeled detection antibodies. Further, the pad must be able to consistently release the detection antibody when the sample fluid flows over it and the analyte binds. The release occurs when the sample solubilizes the antibody, carrying it down the remainder of the assay [13]. Additionally, neither the detection antibody nor the analyte should bind to the conjugate pad, meaning that the pad material, either intrinsically or after pre-treatment, must exhibit low non-specific binding.

\subsubsection{Membrane}

When the fluid has flown across the conjugate pad, it reaches the membrane. The membrane is the bottom later of the lateral flow assay and it contains the detection region of the test, namely, pre-bound capture antibodies. The purpose of this analytical region is to bind the analyte and detection antibody complex from the conjugate pad onto the membrane to allow for quantitative or qualitative test results. Similar to the conjugate pad, the membrane also needs to be able to store capture antibodies for the duration of the test's shelf life [14]. Also, when the test is running, the membrane must facilitate consistent sample capillary flow, allowing the analyte to bind the capture antibody while the other excess buffers, labels, and reactants pass without binding [14]. Typically, the analytic region has two lines: the test line and the control line. Antibodies that are specific to the analyte of interest are immobilized on the membrane test line in a strip and will capture the analyte and labeled detection antibody complex when it flows through the line. If the analyte is present in the sample, it will bind on the antibodies on the test line and the labeled conjugate antibody will visually signify binding with color, indicating a positive result [13]. The control line is used to validate that a sufficient concentration of detection 
antibody was introduced to the assay and that the lateral flow assay functioned correctly. It is created by immobilizing, "species-specific anti-immunoglobulin antibodies, specific for the antibody in the particulate conjugate" [14]. The test line should be designed with sufficient antibodies to bind enough of the conjugated complexes so that a visible signal will be returned, while some complexes flow past onto the control line and bind there, indicating that the test ran properly and the result from the test line is accurate. This reaction can be seen in the diagram below in Figure 4 .

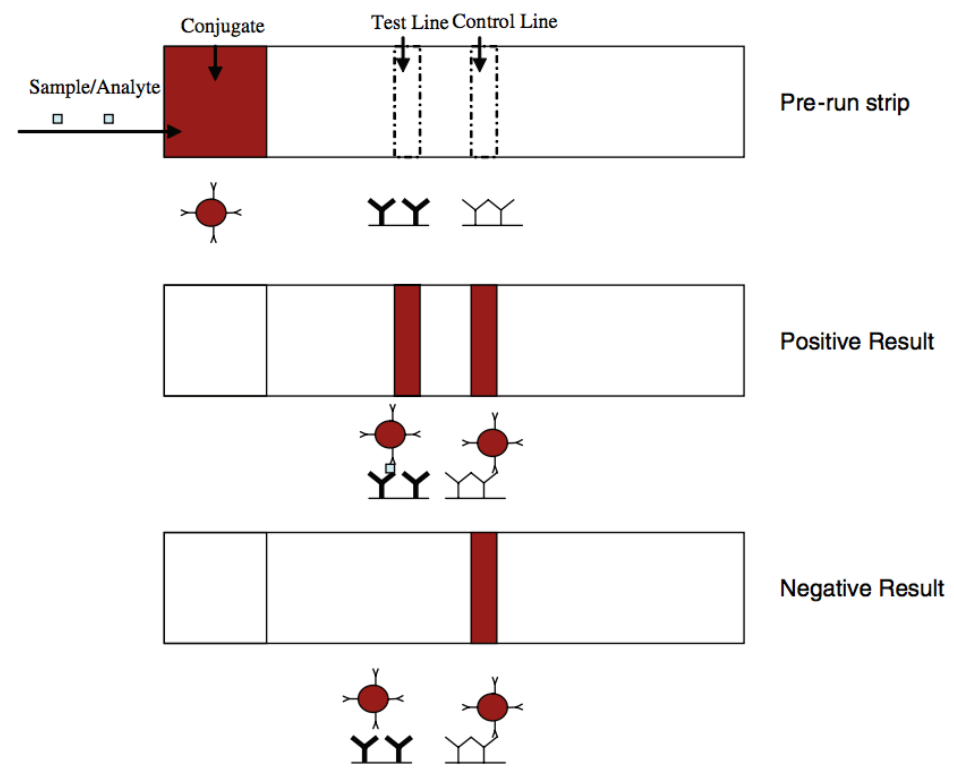

Figure 4. Test and control line antibody loading and colorimetric display [14]

The positive result shows both lines - the test worked, and the device detected the analyte. The negative result shows only the control line - the test worked, and the device did not detect the analyte. If the assay does not run correctly with enough detection antibodies, the assay will display a test line but no control line.

The analytical region of LFAs has historically been nitrocellulose, which is formed by nitrating cellulose through exposure to nitric acid, resulting in the polymeric structure seen below in Figure 5. 


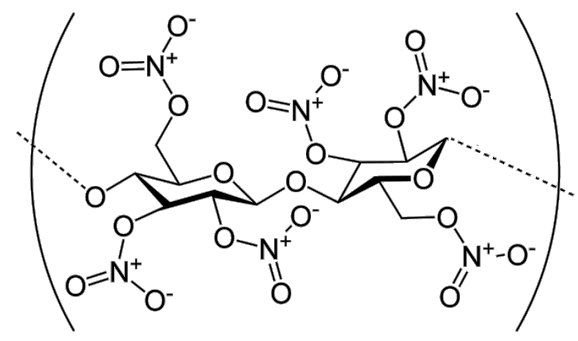

Figure 5. Chemical structure of nitrocellulose [18]

Nitrocellulose is an ideal membrane because of its low cost, true capillary flow characteristics, high non-specific binding of biological macromolecules, relative ease of handling (with direct cast, or backed membranes), and, with specific material treatments and manufacturing processes, varying wicking rates and surfactant contents [14]. Nitrocellulose sheets are typically sold by pore size, ranging from 0.05 to $12 \mu \mathrm{m}$; however, since the pores are not evenly distributed throughout the material, it is more accurate to choose the nitrocellulose using the capillary flow time (seconds/cm) which defined as the time taken for the sample to completely fill the length of membrane [19]. A larger pore size correlates with a lower capillary flow time, and a smaller pore size correlates with a higher capillary flow time [15]. The sensitivity of the assay increases as the flow time decreases because the sample is able to incubate for a long time with the detection molecules.

The other important component of the nitrocellulose is its ability to bind the antibody protein. As seen in Figure 5, nitrocellulose has strong dipoles on the nitrate ester (positively charged nitrogen and negatively charged oxygens) which allows it to electrostatically bind to the antibody and its polar peptide bonds, directly adsorbing the antibody to the surface of the nitrocellulose [16h]. Other methods include covalent attachment of thiolated affinity protein to GMA-modified nitrocellulose, anchoring of biotinylated affinity protein to streptavidin, and genetic fusion of affinity protein to custom 
nitrocellulose-binding anchor protein which are useful in increasing the binding ability of the nitrocellulose, particularly when the capture agent is not an antibody [20].

\subsubsection{The Absorbent Pad}

The final component of the lateral flow assay is the absorbent (wicking) pad, seen at the far end of the LFA in Figure 3. The function of this pad is to create void space for the sample to flow past the test and control lines, allowing sufficient accumulation while the assay reaction occurs. [14]. In general, the total volume of sample that the assay can handle is controlled by the bed volume of the absorbent pad, therefore, a pad with a bed volume large enough to accommodate both the sample and reagent buffer volume [15]. The absorbent pad is typically cellulose. The same considerations that were made for the sample pad apply for the absorbent pad.

\subsubsection{Device Housing}

The final component of the lateral flow assay is the housing, which encases the device, holds the various pads together, and applies regions of pressure at precise locations to ensure connectivity and aid in fluid routing. The housing should be designed to expose the sample pad and allow for viewing of the analytical region where the test and control lines will appear after the test has completed [14]. Depending on the design, adhesive may be used in addition to the housing to hold the assay layers together. The housing design needs to be carefully considered because the connectivity of the assay material layers is reliant on the pressure from the housing and any internal bars or pins which work to hold the materials in contact as well [14]. Due to this, it is important to consider the thicknesses 
of each of the pads because any variation will create a variation in the pressure from the housing - thin layers resulting in little to no contact and thick layers resulting in over compression [19].

\subsection{Paper-Based Microfluidic Assays}

A relatively new technology in the assay space is the paper-based microfluidic assay (referred to as $\mu$ PADS) which uses the chemical reactions seen in lateral flow assays and adapts them for use on a paper-based system. Paper is a cellulose fiber web with a high surface area, which is abundant, inexpensive, and compatible with biological samples, making it an ideal platform for the application of assays [21]. In general, these microfluidic devices are made of patterned paper on which a small volume of fluid will move by capillary action and ultimately yield results in a manner similar to the LFA, at a lower cost, while being less technologically complex, and more environmentally friendly [22].

Typically, the paper is a cellulose material for fluid routing and a nitrocellulose material for the detection region, similar to those used in LFAs [21]. These are ideal because they are affordable, naturally hydrophilic, and allow for rapid liquid movement across the surface and through their bulk. The paper is patterned with hydrophobic areas to create barriers that direct the fluid movement both in plane and out of plane (3D) directions, depending on the assay's intended use. The hydrophobic barrier must fill the entire thickness of the paper in order to create a barrier for the channel from which the fluid cannot escape [23]. Various approaches, including cutting, photolithography, plotting, inkjet etching, plasma etching, wax printing, etc., are utilized to create the fluid flow channels, as seen in the cross section in Figure 6 below [24]. 


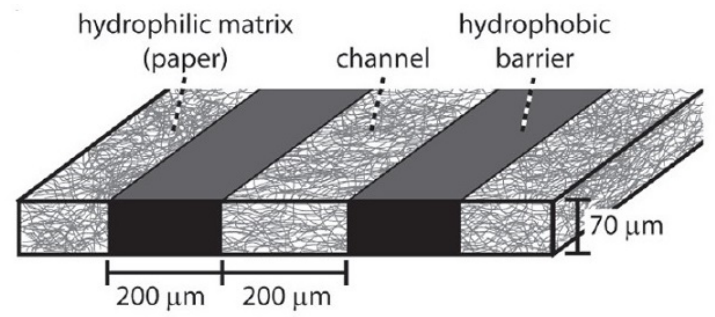

Figure 6. Hydrophilic channel created by hydrophobic barrier [22]

\subsubsection{Photolithography}

The first method developed to create these channels was photolithography, which uses UV light to develop the fluid flow design onto the paper. One example of this process was developed by Martinez, et al. in 2008 in order to rapidly produce assays from novel fluid flow designs, aptly named FLASH (Fast Lithographic Activation of Sheets) [25]. The steps in the creation are outlined below:

(i) Impregnate paper with photoresist and allow it to dry.

(ii) Cover one face of the photoresist-impregnated paper with an adhesive transparency film and the other face with black construction paper.

(iii) Print a pattern onto the transparency film using an ink jet printer, a photocopying machine, or a pen.

(iv) Expose the paper to UV light.

(v) Bake the paper to polymerize the photoresist.

(vi) Remove unpolymerized resist by washing the paper with acetone and isopropyl rubbing alcohol [25].

This novel method was a breakthrough for paper-based microfluidics because it allowed for rapid prototyping at an inexpensive cost with relatively simple materials and processes at an approximate cost of $\$ 26.53 / \mathrm{m}^{2}$. While simple, the method is capable of creating 
hydrophilic channels as small as $100 \mu \mathrm{m}$ wide, and hydrophobic barriers to direct the flow as small as $200 \mu \mathrm{m}$ wide [24]. The fourth and fifth step could be replaced by sun exposure at the cost of pattern accuracy in order to further simplify and cheapen the materials required for the process. Photolithography started a movement to create diagnostic devices that are accessible and cheap, when compared to traditional LFAs.

\subsubsection{Wax Printing}

Wax printing of $\mu \mathrm{PADs}$ arose as an alternative to other more expensive options in 2009 from the groups of Carrilho et al. (2009) and Lu et al (2010) [26][27]. This method is considerably less expensive than photolithography at $\$ 1 / \mathrm{m}^{2}$ and requires fewer materials and a less complex process, making it the most widely accessible method for paper-based assay creation [28]. There are currently three main methods for hydrophobic barrier creation, including: (i) painting with a wax pen, (ii) printing with a normal inkjet printer, followed by tracing by painting with a wax pen (iii) direct printing by a wax printer [28]. The steps for the direct printing method are very simple: first, the barrier design is printed onto the paper using a wax printer and then the wax is melted through the paper using a hot plate, as outlined in Figure 7 below [22]. 


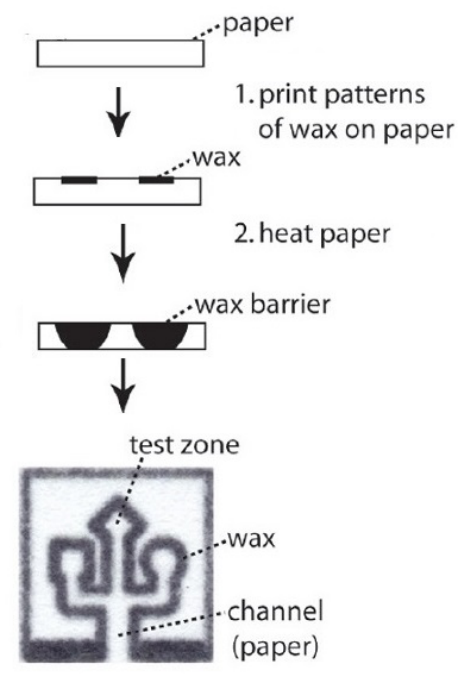

Figure 7. The process of wax printing to allow for fluid flow channels [22]

Typically, this process takes 5-10 minutes from start to completion and is environmentally friendly since the wax and paper can be disposed of via burning. The downfall of wax printing is that during the melting process, the wax melts both downward through the bulk of the paper and laterally across the surface, infringing on the hydrophilic channels [21]. By studying the relationship between applied heat and wax spreading, Carrihlo et al. were able to produce minimum barrier and channel widths of 850 and 560 $\mu \mathrm{m}$, and when applied to nitrocellulose, Lu et al. (2010) were able to create channels as thin as $300 \mu \mathrm{m}$ and $60 \mu \mathrm{m}$ barriers [27].

\subsubsection{Creation of Assay}

In order for the device to be used diagnostically, the hydrophilic path must include a detection zone in which a chemical reaction can occur. This includes an acid-alkali reaction, a precipitation reaction, a reduction-oxidation reaction, or an enzymatic reaction between the target analyte and any immobilized reagents [28]. These reactions produce a signal which can be detected by colorimetric, electrochemical, fluorescent, or a variety of 
other methods, depending on the application of the device [28]. For example, the photolithographic device created by Martinez et al. was used to test for glucose and proteins using well known colorimetric assays commonly used in urine analysis [21]. The glucose test reacted the sample with potassium iodine mixed with horseradish peroxidase and glucose oxidase, which led to the detection zone changing color from clear to brown [23]. Protein concentration was analyzed using citrate buffer with tetrabromophenol blue and colorimetric change from yellow to blue. The different chemicals and enzymes were spotted at the last step of the device fabrication and left to dry in the detection zones, as seen in Figure 8 below [21].

a)
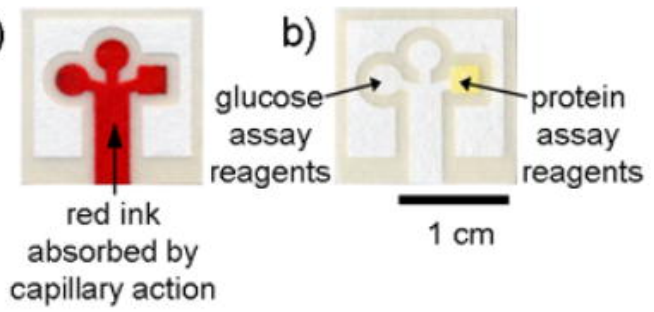

c)

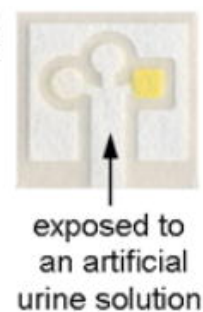

d)

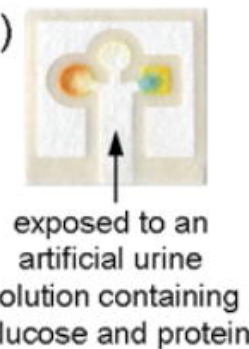

Figure 8. Progression of analytical testing of the paper microfluidic device [23]

Similar to an ELISA, $\mu$ PADS can detect a specific antigen using bound and labeled antibodies and then display a signal using an enzymatic (colorimetric) reaction which is proportional to the quantity of analyte. In a 2012 publication, Wang et al. demonstrated the ability to conduct a complete ELISA on a wax screen-printed chromatography (cellulose) paper using chitosan-modified paper to covalently immobilize antibody and detect specific antigens [29]. However, this device requires a holder specifically created to mount the 
assay, incubate it with the sample and antibodies, and read out the colorimetric results, making it impractical for use outside of the lab, which was the original intention of the paper-based assay [29]. Proving that the chemistries of ELISAs can be implemented on a paper-based device is important to the continuation of this field of diagnostics.

\subsubsection{D Microfluidic Devices}

The examples previously shown of $\mu$ PADS were all single-layer, lateral flow systems. However, the field has progressed to include three dimensional devices which utilize layers of paper stacked upon each other in order to route the liquid in the vertical and horizontal planes. The individual papers are patterned in a way that allows the fluid to flow through the intended path through hydrophilic areas overlapping between the layers [22]. The design must allow for the alignment of hydrophilic areas so that the fluid may continue to flow. Often, these layers are held together using double-sided adhesive tape that are also cut in corresponding patterns to allow for the paper layer conductivity. Since the tape creates a small, but significant, distance between the adjacent layers, a paste made out of cellulose powder and water can be used to fill in the holes cut in the tape to further aid in connectivity [25]. Another method used to create the three-dimensional movement is by using "origami" folding patterns which cause the overlap of paper layers and thereby creating the continuous fluid networks. Alternatively, the layers are held together using specifically designed plastic housing which includes ports which allow for sample input and result read out. 


\subsubsection{ELISA-Like Assay}

Building off of the idea of $\mu$ PADs with lateral flow assay functionality provides the next logical step in the device development: incorporation of ELISA-like functionality onto a paper-based system (P-ELISA) in order to provide the sensitivity and specificity of an ELISA in a cheap, easy to use, and portable system. The main benefit to this inclusion is the fact that ELISAs are run using antibodies which are conjugated to enzymes which cause fluorescence when presented with a substrate [30]. The initial and simplest designs of a PELISA involved the creation of circular portions of cellulose paper made using photoresist, as described above, following the FLASH method designed by Martinez, et al (2008) $[25][30]$.

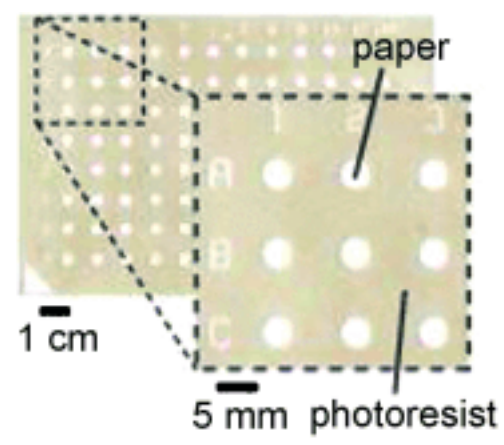

Figure 9. Paper-Based ELISA Format with Photoresist on Paper [30]

Here, the reagents are deposited by hand onto each zone, one at a time, with wash steps between, in order to fully mimic the way that an ELISA would be completed using a 96well plate. These early formats were able to get limits of detection (LOD) significantly lower than that of a standard ELISA and receive results approximately four times faster than an ELISA, all while using a smaller volume of reagents on a cheaper and easier to fabricate platform [30][31]. A downfall of this specific design is that all of the reagents need to be manually placed on to the paper by the end-user, as opposed to coming 
incorporated with the device, as in traditional LFAs, leading to increased likelihood of error when running the assay. Furthermore, the large paper does not have a housing nor is it portable, making it difficult to bring to the end user. This design was improved on further with the creation of sliding P-ELISAs, where a separate paper card was designed and assembled to slide laterally in relation to the test zone [32]. In this iteration, the antibodies and other reagents were stored on the card and resolubilized using water or buffer solutions to allow the sample to mix and deliver a result, as in Figure 10 below.

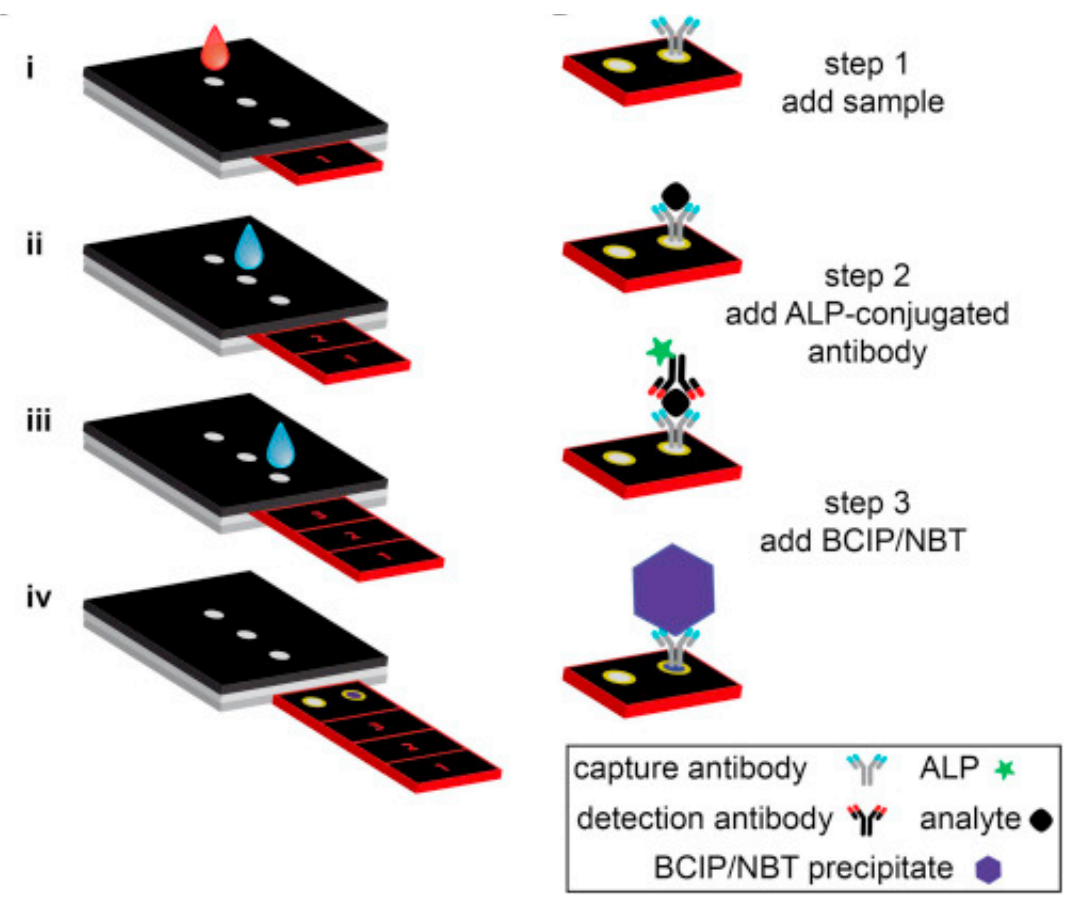

Figure 10. Sliding Format for a P-ELISA Showing the Layers and Reagents [32]

In this format, the sample is added to the first circle, as shown above, and then the subsequent steps are water which is used to resolubilize the proteins bound to the cellulose layers above the sample strip. Finally, the result is read when the strip is pulled the entire way out of the device. This test, while allowing for on board reagent incorporation, only allows for one sample to be tested for one protein at a time. A multiplex assay would allow 
for several biomarkers to be tested for in one sample in order to gain a more useful diagnosis for complex pathologies. Also, since the layers are not seen to the end user, it is hard to line up the test region properly using only the guidelines on the sliding strip [32]. This thesis seeks to improve on the current P-ELISAs by allowing for on board reagents which can specifically and sensitively detect the presence of a biomarker in a multiplex fashion.

\subsection{On Board Reagent Incorporation}

The further development of the paper-based microfluidic assay to include the reagents onboard the device will allow for minimal sample preparation and reduce user error when in a clinical or research setting. Creating a system for on-board reagents thus improves the robustness, affordability and ease-of-use of the device while minimizing the amount of external interaction required, including test equipment [33]. Because the paper platform is porous, it has the ability to store liquids, such as assay reagents, that would typically be separate and added manually to the assay [34]. These reagents will resolubilize once the sample is added to the device and flows past the dried down reagent. The process for adding the reagents is straightforward; the reagent is either spotted on the paper or the paper itself is fully submerged in the reagent. Then, the paper is left to dry and incorporated into the device and left until the device is used, similar in concept to the lateral flow assay. The main challenges faced with reagents in paper-based devices is the stability of the reagent, which relies on careful temperature control, and the controlled release of the reagents so that the sample has sufficient time to interact and react [28][33]. After loading the reagent onto the pad, storage at colder temperatures is desired as it maintains the activity 
of the antibodies, however, signal has been seen to only minimally decrease when stored at room temperature $\left(20^{\circ} \mathrm{C}\right)$ and hot temperatures $\left(45^{\circ} \mathrm{C}\right)$, as seen in Figure 11 below.

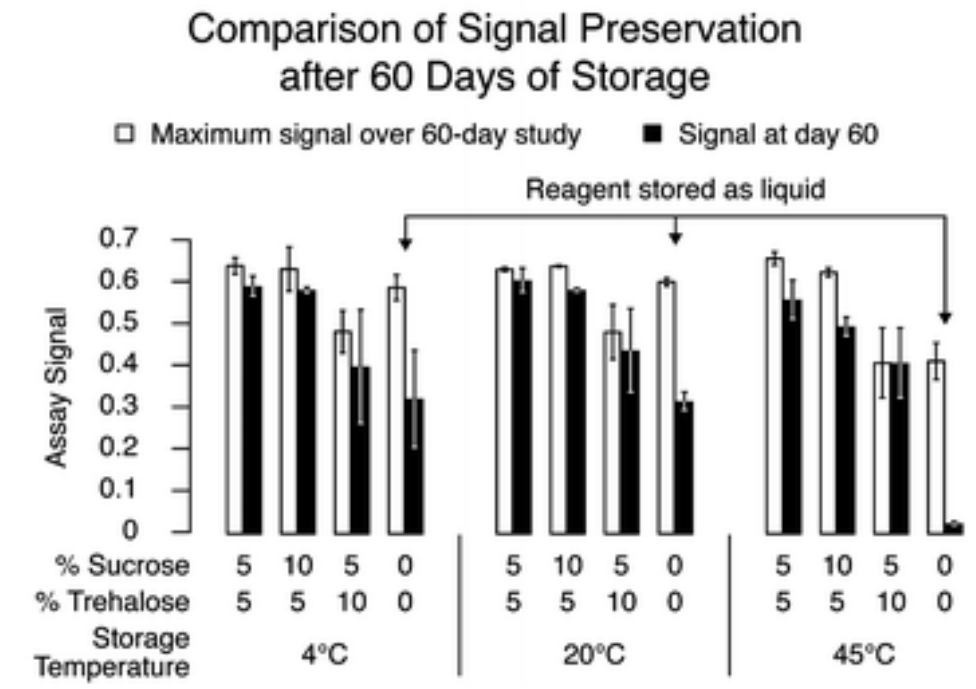

Figure 11. Chart showing the effect of long-term dry storage of the conjugate on assay performance [Stevens]

The conjugate antibody was preserved with a sugar addition at varying percentages, as shown above, in order to determine if there was an effect on storage temperature and conditions on the resulting signal [35]. When comparing the temperatures at 5\%/5\%, there is a clear, but small, decrease when stored at $45^{\circ} \mathrm{C}$ for 60 days, however, the $4^{\circ} \mathrm{C}$ and $20^{\circ} \mathrm{C}$ storage temperatures did not show much of a decay in signal. This means that the device could be stored at any temperature, most preferably around $20^{\circ} \mathrm{C}$, and maintain its functionality [35]. Dried reagents would be resolubilized with buffer, such as PBS, for delivery to the sample. The incorporation of these reagents on board work to minimize user steps, remove the need for a cold chain, and create nearly automated devices [33]. 


\subsection{Characteristics of Fluid Flow in Paper Microfluidics}

Paper-based microfluidic devices can entirely and autonomously run on capillary action, which occurs as the result of the intermolecular attraction within the liquid and solid materials [34]. The liquid is able to flow in narrow spaces without external input of energy due to the imbalance of adhesive forces between the liquid and the material and the cohesive forces within the liquid itself [36]. The liquid begins to move when the adhesive forces are greater than the cohesive forces. Typically, capillary flow is understood as the flow of liquid through a small cylinder, which can be determined using Poiseuille's law, equation 1 below:

$$
Q=\frac{\Delta P \pi r^{4}}{8 \eta l}
$$

Where the flow rate, $\mathrm{Q}$, is determined by the pressure gradient in the tube, $\mathrm{P}$, the radius of the rube, $r$, the length of the tube, 1 , and the viscosity of the fluid, $\eta$. This relationship only applies to the laminar flow of an incompressible liquid, however, when integrated with respect to time and inclusive of the total driving pressure with the capillary open at both ends, the resulting equation is descriptive of the flow of liquids under its own capillary pressure [36]:

$$
L^{2}=\frac{\gamma D t}{4 \mu} \cos \theta
$$

where $\mathrm{L}$ is the distance moved by the fluid front, $\mathrm{t}$ is time, $\mathrm{D}$ is the average pore diameter, $\gamma$ is the effective surface tension, $\cos \theta$ is the contact angle dependence, and $\mu$ is viscosity. This equation is known as the Lucas-Washburn equation and can be extrapolated to fluid flow through porous media by taking the penetration of the pores by a liquid as equal to the penetration of $n$ cylindrical capillary tubes of varying radii [36]. Per the understanding of capillary flow, the force due to the surface tension resulting from the adhesive force 
between the liquid and the pores pulls the fluid further into the paper [37]. With simplification, as shown above, Washburn-like flow is characterized by $\mathrm{L} \sim \sqrt{t}$, which implies that the fluid front velocity decreases with time. As the surface tension is pulling the water into the paper's pores, the viscous resistance slows the fluid front down, resulting a decrease in flow velocity of the fluid front as it enters the porous media [37].

\subsubsection{Wicking}

When specifically referring to capillary flow within a porous media, wicking is the action of spontaneous absorption that occurs [38]. While the Washburn equation is often sufficient enough to estimate this action, it has distinct shortcomings with ignoring the idea of a preferred pathway of flow through a medium with non-homogenous pores [39]. Namely, pores with a relatively small diameter will fill faster than those with larger diameters, thereby advancing the fluid front up the medium through adjacent small pores at a faster rate than the large pores [39]. Additionally, the Washburn equation is limited to one dimensional wicking due to the original approximation using capillary tubes [38]. In order to adjust for these shortcomings, Darcy's Law can be used:

$$
Q=-\frac{\kappa A}{\mu L} \Delta P
$$

where $\mathrm{Q}$ is the volumetric flow rate, $\kappa$ is the permeability of the paper to the fluid, $\mu$ is the viscosity of the fluid, $\mathrm{A}$ is the area of the channel perpendicular to flow, and $\Delta \mathrm{P}$ is the pressure difference along the direction of flow over the length L [37]. Darcy's law applies to fully-wetted flow, where the fluid front has reached the wicking pad and the velocity of the fluid flow is approximately steady [37]. Pressure can be estimated using only the 
definition of capillary pressure, below, because the atmospheric pressure difference in the device is equal to zero.

$$
p_{c}=\frac{2 \gamma \cos \theta}{R_{\text {pore }}}
$$

Where Eq. 4 can then be substituted in to Eq. 3 for the volumetric flow rate in a series of capillaries taken as equivalent to a porous media. Dividing by area results in Eq. 5 below, the velocity of fluid flow through cellulose paper with consideration of wicking and capillary flow.

$$
v=-\frac{\kappa}{\mu L} \frac{2 \gamma \cos \theta}{R_{\text {pore }}}
$$

The continued development of equations to model fluid flow characteristics in porous media is important to understanding the variables that can be changed for components of the paper-based microfluidic devices in order to control the flow rates and achieve optimal results in both lateral and 3D microfluidic devices.

\subsection{The Diagnosis of Stroke}

A stroke occurs when there is an interruption to the flow of blood in the brain, due to either a physical blockage or a broken blood vessel. Strokes are divided into two categories: hemorrhagic and ischemic. Hemorrhagic strokes are due to a blood vessel bursting due to pressure or stresses at the vessel wall, allowing blood to leak into the brain, creating swelling and pressure which, in turn, damages the neural cells and tissue [40]. Ischemic strokes occur when a blockage is formed in or travels to a cerebral blood vessel and the flow of blood is stopped to that area of the brain. The blockage can be caused in several ways, including the atherosclerotic buildup of fat and plaque. Further, they can be 
characterized as a thrombotic stroke, where a blood clot formed at the stroke site, or as an embolic stroke, where a blood clot traveled through the vasculature from elsewhere in the body until it was stopped by a small enough vessel [41]. Regardless of the cause, a lack of blood to the brain tissue leads to hypoxia and irreversible cell death.

Hemorrhagic strokes are treated in a variety of different ways, all for the main goal of stopping the brain bleed. Primarily, doctors will attempt to lower the patient's blood pressure using medication or a ventilator. Later, a surgical clip may be placed at the base of the brain aneurysm to remove blood flow and stop the bleeding, an invasive procedure which requires a portion of the skull to be removed [42]. Ischemic strokes are often treated using tissue plasminogen activator (tPA) which is a protease found on endothelial cells that breaks down clots [43]. Due to the extreme nature of stroke and relatively quick damage to the brain, the earlier a stroke is caught and diagnosed, the better the patient outcome. Correct diagnosis is vital because the anti-coagulation medication given to ischemic stroke patients would be deadly to hemorrhagic stroke patients as the ruptured vessel would be unable to clot and mitigate blood flow.

In emergency rooms today, a CT scan is the standard for assessing the presence and extent of neurological trauma. This test can show areas of abnormalities in the brain and can help to determine if these areas are caused by ischemic stroke, hemorrhagic stroke, or a different kind of a problem [44]. Unfortunately, without contrast solution, CT scans miss ischemic strokes, which account for $\sim 80 \%$ of strokes [45]. Therefore, a stroke will not be diagnosed efficiently, which would increase the risk of long-term complications and even death. 


\subsubsection{Biomarkers}

During and after a stroke, there are detectable macromolecular signals, biomarkers, in the blood stream that can be used to diagnose the disease [4]. These molecules must be able to be detected and correlate to ischemic stroke in a repeatable, reliable, and accurate manner. Currently, several protein biomarkers are considered indicative of ischemic stroke, including C-reactive protein, lipoprotein-associated phospholipase A2, and urinary albumin excretion [46]. Other biomarkers have been well studied in stroke including natriuretic peptides, glial fibrillary acidic protein (GFAP), S100b, neuron specific enolase (NSE), myelin basic protein (MBP), interleukin-6, matrix metalloproteinase (MMP)-9, Ddimer, and fibrinogen [46]. The problem with using biomarkers to detect and diagnose stroke is that each stroke is heterogeneous and has large variability in location, size, and cause, therefore some assays may not include the specific biomarker that a patient suffering from stroke is producing in their bloodstream. Therefore, researchers are seeking out a something akin to a "mosaic" of biomarkers, or a protein panel, which together lead to a better and more accurate diagnosis than they would separately. In order for a complete and highly accurate diagnosis, one device would need to be able to indicate the presence of a variety of biomarkers that have all been contraindicated to ischemic stroke. In a comprehensive study of published ischemic stroke biomarker panels, Jickling and Sharp (2015) compiled the results of several studies on ischemic stroke detection, specifically in regard to the proteins used and the reported sensitivity and specificity [46]. A brief selection of that information can be found in Table I below. 
Table I. Biomarker Panels for the Diagnosis of Ischemic Stroke [46]

\begin{tabular}{|c|c|c|c|c|c|}
\hline Panel & Comparison & $\begin{array}{c}\text { Assay } \\
\text { Platform }\end{array}$ & $\begin{array}{c}\text { Sample } \\
\text { Size }\end{array}$ & $\begin{array}{c}\text { Markers } \\
\text { in Panel }\end{array}$ & $\begin{array}{c}\text { Sensitivity/ } \\
\text { Specificity }\end{array}$ \\
\hline $\begin{array}{c}\text { Five } \\
\text { protein } \\
\text { panel }\end{array}$ & $\begin{array}{c}\text { Ischemic }+ \\
\text { hemorrhagic } \\
\text { stroke vs } \\
\text { control }\end{array}$ & ELISA & 437 & $\begin{array}{c}\text { BNGF, } \\
\text { MCP-1, } \\
\text { MMP-9, } \\
\text { S100B, } \\
\text { vWF }\end{array}$ & $92 \% / 93 \%$ \\
\hline $\begin{array}{c}\text { Four } \\
\text { protein } \\
\text { panel }\end{array}$ & $\begin{array}{c}\text { Ischemic }+ \\
\text { hemorrhagic } \\
\text { stroke vs } \\
\text { control }\end{array}$ & ELISA & 1,289 & $\begin{array}{c}\text { BNP, D- } \\
\text { dimer, } \\
\text { MMP-9, } \\
\text { S100B }\end{array}$ & $86 \% / 37 \%$ \\
\hline $\begin{array}{c}\text { Four } \\
\text { protein } \\
\text { panel }\end{array}$ & $\begin{array}{c}\text { Ischemic } \\
\text { stroke vs } \\
\text { control }\end{array}$ & ELISA & 222 & $\begin{array}{c}\text { MMP-9, } \\
\text { S100B, } \\
\text { VCAM1, } \\
\text { vWF }\end{array}$ & $90 \% / 90 \%$ \\
\hline
\end{tabular}

Of note in this table is the fact that the panels were comprised of a set of either four or five proteins which together gave high values for sensitivity and specificity, in the case of the first and last panel, yet gave low specificity in the middle panel. This indicates that the specific protein makeup of the panel has a large influence on the success of the diagnosis and changing a few biomarkers can have a marked result in the test specificity.

Furthermore, this subset of data also only looked at ischemic and ischemic + hemorrhagic stroke populations versus control populations, rather than ischemic stroke versus hemorrhagic stroke. It is simpler to differentiate a stroke patient from a healthy patient because there would be a greater number of biomarkers which would be significantly expressed differently in the stroke patient versus the control. When comparing ischemic and hemorrhagic stroke, the protein differences between the two pathologies 
become more indiscernible. Stroke biomarkers, in general, appear as the result of brain tissue damage, inflammation, endothelium damage, and coagulation/thrombosis that is occurring with either type of stroke, hemorrhagic or ischemic [46]. This is further muddled by the fact that stroke biomarkers are also elevated in response to other conditions, such as traumatic brain injury, severe myocardial infarction, and brain infection [47]. Additionally, adding to the challenge of using biomarkers for stroke, the blood-brain barrier slows the diffusion of proteins into the blood stream, making it difficult to obtain a sample of blood with the sought-after biomarkers.

$\mathrm{S} 100$ beta (S100B, or S100ß) is a calcium-binding protein expressed by the human body in response to central nervous system injury [48]. This protein has been associated with ischemic stroke in several published articles which analyzed both the physiological response to stroke as well as the accurate diagnosis of the condition [46] [48] [49]. For the scope of this thesis, S100B will be acting as the target antigen in the patient sample in order to diagnose an ischemic stroke.

\subsection{A Global Approach to Diagnostic Medicine}

As the understanding of the cellular mechanisms within the human body advances, so does biomedical technology. This leads to new methodologies for detection and diagnosis of diseases, infections, and other pathologies which were previously unknown. These detection methods are becoming more sensitive and are able to detect a larger variety of molecules with higher accuracy. Developing these advanced techniques across all fields of medicine and diagnostics comes with years of research and design which is often very

costly. These technologies are often complex and expensive to manufacture and require the 
user to have a specific, trained skillset in order to operate the device. Therefore, gains have been made in the point-of-care (POC) space, with the design goals of cheap and disposable devices that can be used in almost any space with small amounts of experience. These POC devices allow a patient to get accurate results in a reasonable time frame without the need for a visit to a large clinic or hospital, which may be unattainable in some areas. Further, these devices are portable and could be taken into remote areas where people would benefit from access to diagnostic medicine for pathologies such as malaria and HIV. Therefore, the creation of paper-based $\mu$ PADs, as described above, are a cheap, easy, and fast diagnostic method will allow for the widespread detection and characterization of diseases which can be detected using specific biomarkers. Often times, these devices are forced to sacrifice sensitivity in order to work in the environments seen in developing countries due to the lack of equipment and efforts to make the devices low cost [50]. Dry-form reagent storage on a paper-based microfluidic system could lead to the future of POC diagnostics, allowing sensitive and specific diagnoses for people across the globe.

With this in mind, the World Health Organization (WHO) via the Sexually Transmitted Diseases Diagnostics Initiative (SDI) has created a series of guidelines for the quality of a diagnostic test that would be used in developing countries [51]. The ASSURED criteria are as follows: Affordable, Sensitive, Specific, User-friendly, Rapid and robust, Equipment-free and Deliverable to end-users. These guidelines are meant to be a benchmark to determine if the test meets the needs of the community it would be servicing. It is important that throughout this thesis project, the ASSURED guidelines are considered so that an eventual implementation into developing countries could be feasible. 


\subsection{Dissemination of Previous Work}

The Cal Poly Biofluidics lab has been doing research on $\mu$ PADS since 2012 under the guidance of Dr. David Clague. Clayton (2012) and Ward (2012) designed and characterized several formats of paper-based microfluidic platforms using nitrocellulose [52] [53]. Clayton (2012) compared anti-VEGF antibodies and aptamers on paper microfluidic-based platforms, centered around creating a device to detect the VEGF protein using antibodies and aptamers, or segments of DNA specifically designed to target a protein, similar to a paratope on an antibody. Notable results included the determination of an optimal channel width of $1.6 \mathrm{~mm}$, the confirmation of the homogeneity of fluid flow through nitrocellulose paper and, conversely, the heterogenicity of fluid flow through cellulose paper [52]. Similarly, Ward (2012) developed a nitrocellulose paper-based microfluidic platform with surface functionalization using anti - IgE aptamers. This research determined a channel width of $1.6 \mathrm{~mm}$ in order to create optimal fluid flow times for the lateral flow assay that was used to detect IgE using aptamers [53]. Tageson (2013) brought the technology into 3D applications, routing fluid in the lateral and vertical directions to allow for a series of reactions for binding the $\operatorname{IgE}$ analyte to the aptamer for detection using fluorescence [54]. Young (2016) created a multiplex 3D assay device using a similar method in order to detect glucose presence in a sample via a 3-D multiplex papermicrofluidic platform, which did not deal with an antibody/antigen immunological reaction but did further validate the 3D functionality of wax-printed, paper-based assays [55]. Most recently, Holler (2016) continued the work of Tageson (2013) and was able to design a rotating paper-based $\mu \mathrm{PAD}$ using wax printing which had the theoretical ability to carry out a functional ELISA-like assay [56]. However, in the work, they were unable to fully 
demonstrate a device that used on board reagents, i.e. the inclusion of the detection antibody on the conjugate pad [56].

In separate, but related, work, Daugherty (2015) created code that could determine absorbance values of an assay which used gold-labeled nanoparticles to quantify a signal [57]. Van Huynh (2016) further created MATLAB code that quantifies color intensity of an HRP-bound antibody on cellulose with a smart phone image [58]. Both Daugherty and Van Huynh were able to obtain statistically valid results of color intensity using their respective code run on a cell phone image of a completed assay [57] [58].

Moving forward with my own thesis, I will build off of the work done by Alicia Holler in the creation of a 3D paper-based, microfluidic device that is built to enable an ELISA-like assay for the detection and diagnosis of ischemic stroke. 


\section{MATERIALS AND METHODS}

The process of the creation and associated testing of the 3D microfluidic chip $(\mu \mathrm{PAD})$ and housing will be explained in this section. This device was built upon previous work completed by Holler (2016) with the initial attempt to incorporate an ELISA-like assay onto a paper microfluidic chip by successfully characterizing fluid flow through a 3D chip design. However, that work was unable to achieve reagent loading to accurately test for a specific antigen [56]. With the design in mind, a series of experiments were conducted to initially determine the fluid flow characteristics of the device and to ensure the connectivity between layers. Then, the device was redesigned to incorporate the desired features that would be required for an ELISA before proceeding with the biological implementation of an ELISA-like test onto the paper platform. Then, the onboard reagents were incorporated into the device to specifically test for the presence of the S100B protein. These reagents were optimized for specificity and sensitivity of the assay as well as consideration of cost, ease of manufacturing, and overall ease of use for the end consumer.

\subsection{D Paper Microfluidic Chip Fabrication}

Various iterations of designs were created in order to determine the optimal layout and sizing for each layer of the device based on previous work, published literature, and further development from optimization tests. AutoCAD ${ }^{\mathrm{TM}}$ was used to design and develop

the layers for the $\mu \mathrm{PAD}$. In general, each design was fabricated, as described below, based on a standard method employed in several past theses. 


\subsubsection{Wax Printing}

The $\mu \mathrm{PADs}$ were created using the wax printing technique similar to that described in the introduction. By designing the layers on AutoCAD ${ }^{\mathrm{TM}}$ and setting the wax-printed areas to a black hatch pattern, the fluid flow pathway could be created, eventually allowing for reactivity and detection. Whatman ${ }^{\mathrm{TM}} 1 \mathrm{~mm}$ thick, 20 x $20 \mathrm{~cm}$ chromatography Paper (CAT No. 3001-861) was purchased from GE Healthcare Life Sciences and was used as the cellulose material for the fluid routing layers of the device. Whatman ${ }^{\mathrm{TM}} 3 \mathrm{~mm}$ thick, 20 x $20 \mathrm{~cm}$ chromatography Paper (CAT No. 3030-861) was purchased from GE Healthcare Life Sciences and was used as the cellulose material for the absorbent pad. Glass fiber conjugate pad (GFCP103000) was purchased from EMD Millipore and was used as a conjugate pad. Unbacked HiFlow Plus nitrocellulose (HFB135UB) was purchased from EMD Millipore for use as the detection layer.

The designs were printed from AutoCAD to a Xerox ${ }^{\circledR}$ Phaser ${ }^{\circledR} 8560$ printer with Xerox ${ }^{\circledR}$ black solid ink. Once the layers were printed, they were individually placed on a Clarkson IKA® C-MAG HP 10 digital ceramic hot plate at $120^{\circ} \mathrm{C}$ for two minutes until the wax was melted through the cellulose and could be seen on the bottom side. The even heating was aided with a pair of metal tweezers used to press down on the paper to ensure complete melting. After melting, each layer was cut out appropriately using a McGill Punchline 3/16" round hole punch. The detection layer was attached to the top layer using 3M $\mathrm{M}^{\mathrm{TM}}$ Double Coated Tape 415 . At this point, the device was assembled, layer by layer, to create the final $\mu \mathrm{PAD}$. 


\subsubsection{Housing Fabrication}

As with any paper-based device, a housing is required to keep the layers oriented correctly and to apply even pressure throughout to ensure connectivity. The housing must also be able to deliver the sample to the sample pad and also display the results of the test, as well as accommodate for end-user functionality and ease of use. AutoCAD was used to design the housing components. The housing design was then laser cut into acrylic sheets of varying thicknesses using a Universal Laser System PLS6.150D platform. The pieces were held together using screws, as described in a later section.

\subsection{Analysis of Previous Work}

Initially, all tests were performed on cellulose paper to establish fluid connectivity on a cheap material with controlled characteristics. The previous design created by Holler (2016) was opened on AutoCAD and printed at a 1:1 scale. The design can be seen in Figure 12 below. Dimensions for this design were based on previous work by Tageson (2013) and Ward (2012) [53] [54]. A dimensioned drawing can be found in the Appendix. 


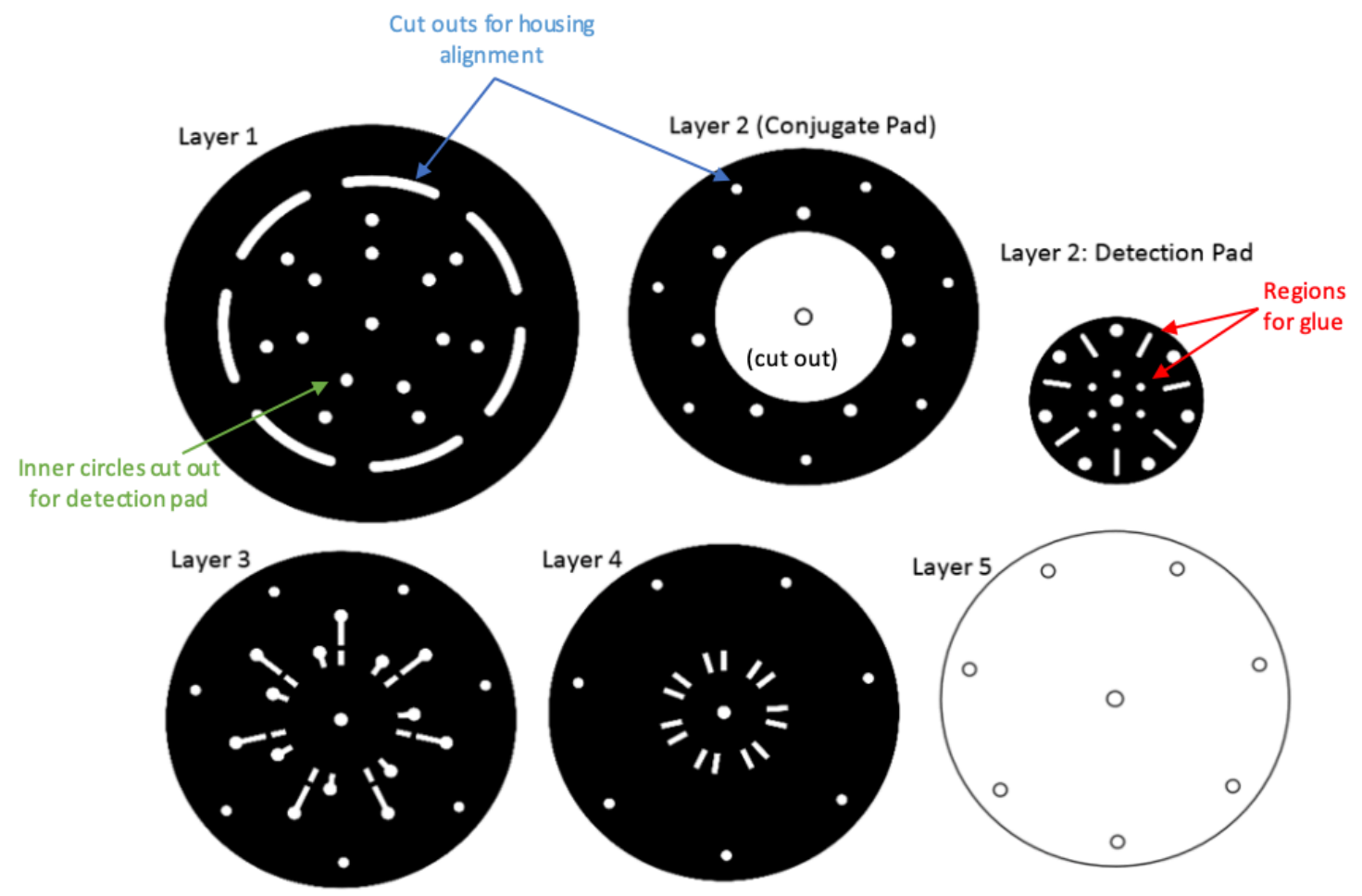

Figure 12. Previous final chip design from Holler (2016) [56]

This design incorporated five layers in total. Layer two was comprised of two individual pieces - the conjugate pad and the detection pad. These layers would be glued together and then, as a unit, glued to the underside of the sample pad, layer one. Layers three and four would be used as fluid-routing and layer five served as the absorbent pad to cause the wicking action through the device. The principle of this design is the pin-wheel action achieved by fixing all of the layers together in the center with a screw and allowing layer one to rotate independently of the exterior screws with arched slots along the exterior that would be cut out after printing. This rotation would open up new channel pathways that could be used for further reagent incorporation and/or wash steps. In this design, there is one main channel and one additional channel used after rotating the top layer. 
The previous work also included production of a housing unit, seen below in Figure 13, which was also analyzed. Dimensioned drawings can be found in the Appendix.
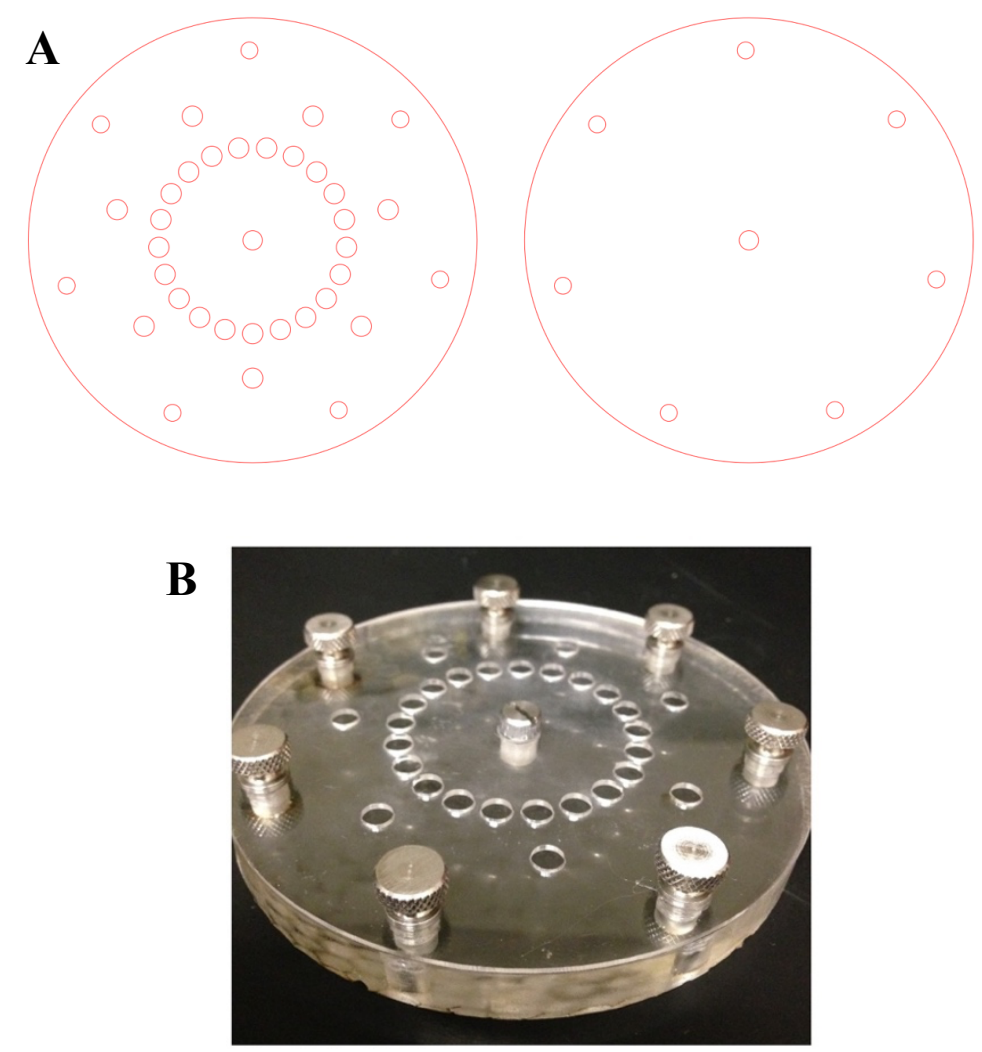

Figure 13. Previous final housing from Holler (2016). A) AutoCAD design, top of housing on left, bottom on right B) Assembled housing [56]

This housing unit was designed in AutoCAD ${ }^{\mathrm{TM}}$ and laser cut as described in Section

2.1.2. The layers were held together by inserting 0.266 " diameter hex threaded inserts into the bottom layer using a benchtop press. The two layers were then held together with \#832 thread size, $3 / 8$ " length screws.

In order to get an idea of the functionality of the device as described in the previous thesis, this design was printed as described above and inserted into the housing. Red dye was created by dropping one drop of red Kroger ${ }^{\circledR}$ Assorted Food Colors ${ }^{\circledR}$ into $15 \mathrm{~mL}$ of distilled water. This dye solution was used to determine if the fluid routing was functional, 
specifically that the fluid would wick through all of the layers, appearing on the detection pad and the absorbent pad to indicate a complete test.

\section{3 $\mu$ PAD Design Evolution}

Based on the results from the previous thesis, the basic concepts behind the $\mu \mathrm{PAD}$ were kept in subsequent novel designs as a part of this thesis. This includes the wax printed circular layers which enable fluid routing in the lateral and vertical directions and can rotate to create connectivity to new channels to allow for wash steps and the addition of off board reagents. Dimensions for all drawings can be found in the Appendix. The first design iteration was based on the previous design, however it had the direction of flow inverted, i.e. the old design flowed sample from ports on the outside inward and the new design placed the sample ports on the inside and flowed fluid outward, as seen in Figure 14.
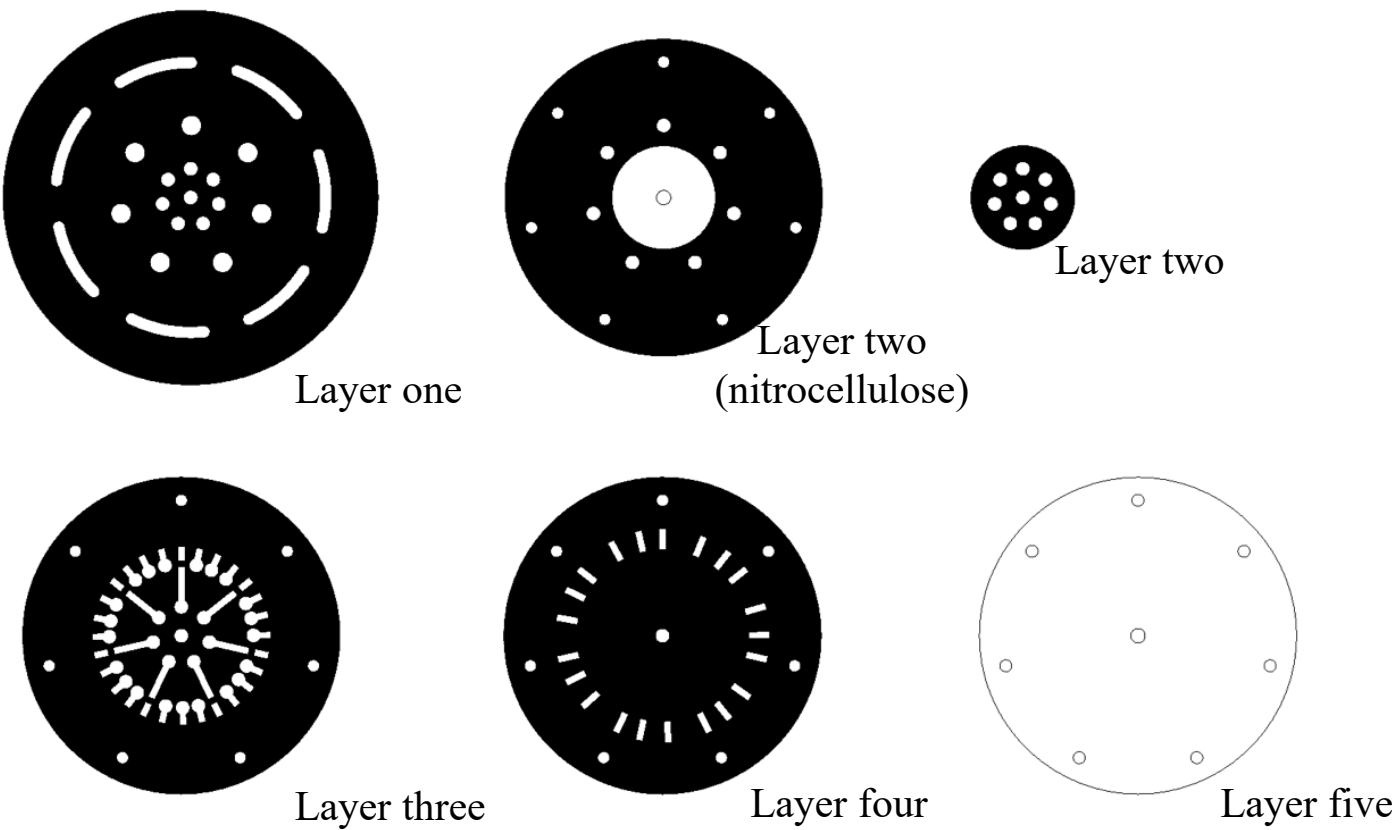

Figure 14. Initial rotational outward flow design 
The long channels would be used to deliver the sample to the detection region. Then, the device would be rotated to align with the first new channel, allowing for a wash step to remove unbound antibody. Then, the test would be rotated again for an additional wash step. Finally, the test would be rotated a final time to allow for the addition of the substrate to indicate if there was a positive result. Similar to the rest of the layers, the smallest circle in layer two was designed to be made of cellulose, while the corresponding ring-shaped portion of layer two was to be printed on nitrocellulose. These two portions of layer two were designed to be taped together and then taped to the bottom of layer one to allow for rotation of the detection pads to the new channels.

Additionally, a new format for the device was drafted, which used lateral motion as opposed to rotational motion, to expose new channels. This design can be seen below in Figure 15.

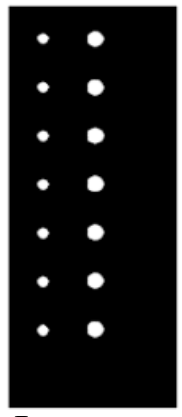

Layer one

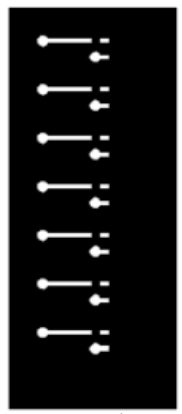

Layer three

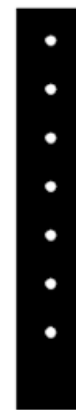

Layer two

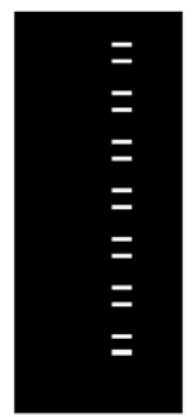

Layer four

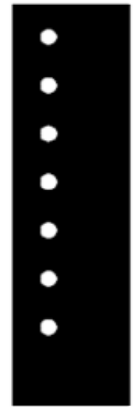

Layer two (nitrocellulose)

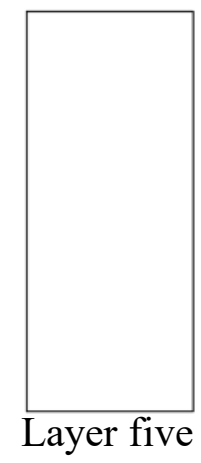

Figure 15. Lateral motion pull strip design 
The basic concept behind this design was to take advantage of the sliding motion which could be achieved with a separate nitrocellulose strip, as in the device designed by Verma, et al. described in the Introduction [32]. To improve on their idea, the device would allow for a standard curve for quantification of the antigen based on a color change. Layer two would still be split into two - one portion cellulose and one portion nitrocellulose to allow for the creation of a detection pad. The nitrocellulose portion would then be pulled through the assay, exposing subsequent channels for additional wash steps. Because this design is linear rather than circular, it would be easier to add more sample channels and wash channels since it would simply require adding those portions to the bottom of the strip, rather than expanding the diameter of the circle of the rotational design to create more space. It was decided to move ahead with the rotational design over the lateral motion design due to the simpler housing and perceived ease of production for the rotating design.

The outward flow design went through several series of design changes based on the evolving requirements for the ELISA functionality. First, the design was changed to include a fourth fluid channel and an area which could function as a control pad - similar to that in a lateral flow assay - which would show if the test ran correctly. In order to accommodate for this, the entire series of channels was expanded outward to create more space between each lane that could fit the maximum number of additional channels. This design can be seen in Figure 16. 

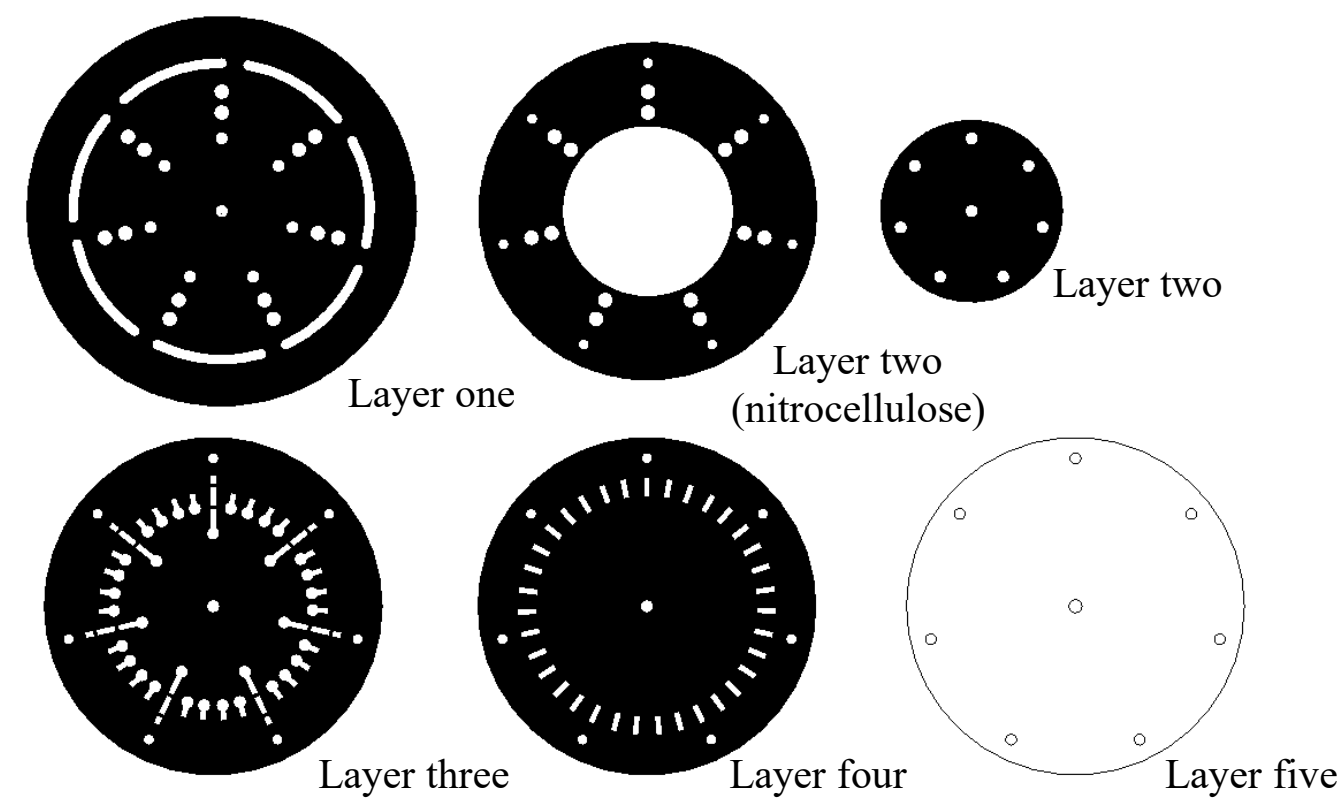

Figure 16. Outward flow design with four channels and a control pad

This design was created to maximize the quantity of additional reagents which could be added to the device - specifically increasing the potential amount of wash steps. As stated, a main reason why ELISAs are able to be sensitive is due to wash steps which remove excess proteins and reagents from the test area and only keep the ones which return a positive accurate result. The control pad is seen in layer one as the third ring of circles from the inside and correspondingly on layer two in the second ring of circles from the inside. It was further determined that the control pad would require its own wash steps as well in order to correspond appropriately to the color intensity of the detection pad and wash away unbound antibody for an optimal signal. Additionally, the number of additional channels was decreased to three because further research indicated that two wash steps and one wash with the substrate would be sufficient. This was added into the next design, seen in Figure 17. 

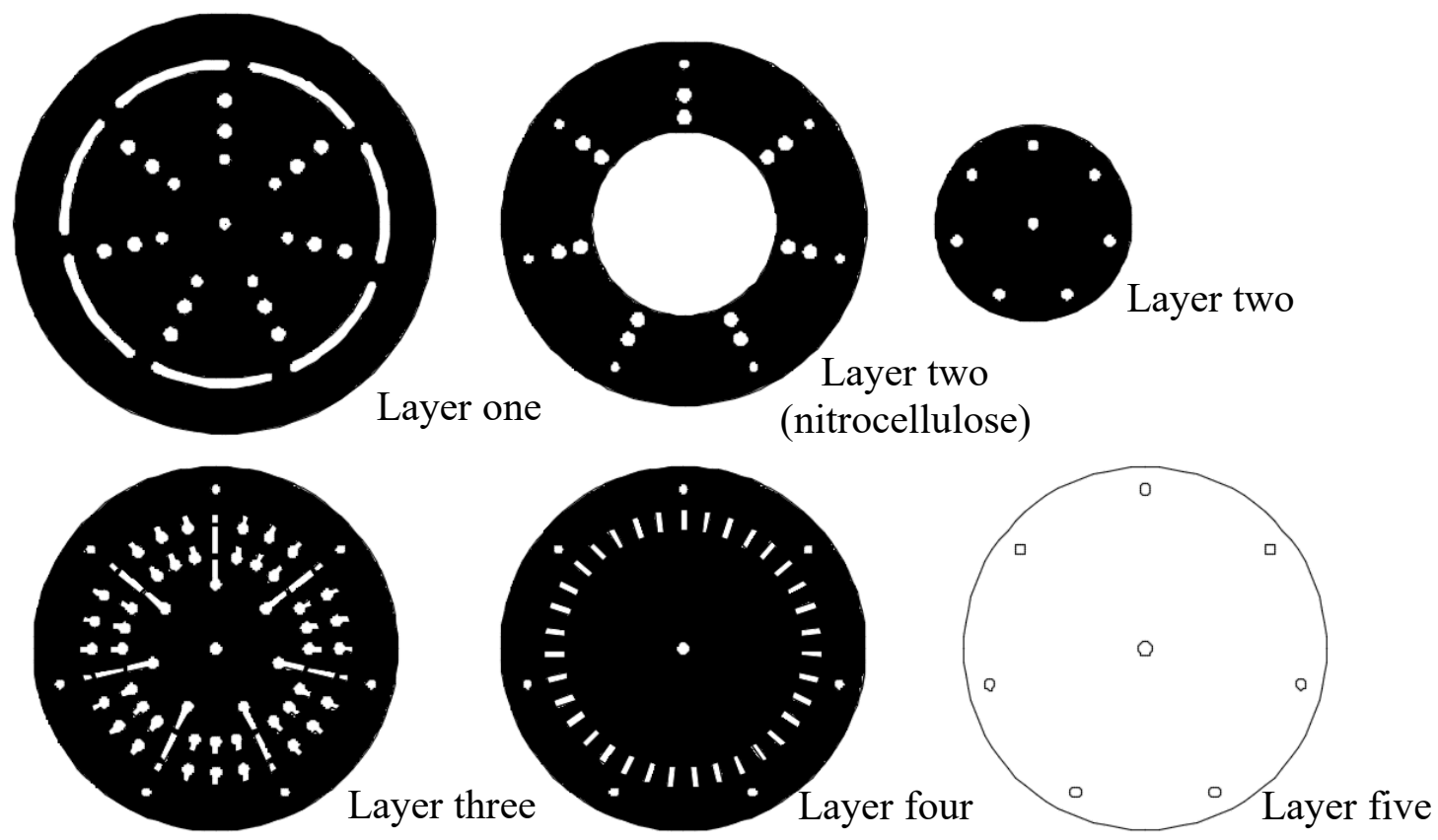

Figure 17. Outward flow design with three detection pad channels and three control pad channels

At this point, the overall design of the product was relatively stable. This led to the desire to determine overall functionality of the layered $\mu \mathrm{PAD}$ system as far as reagent flow is concerned. Before diving into the possibilities that could be explored within the rotational design, a lateral single channel test was created in order to validate fluid flow characteristics using red dye. The design for this test strip can be seen in Figure 18 below. 


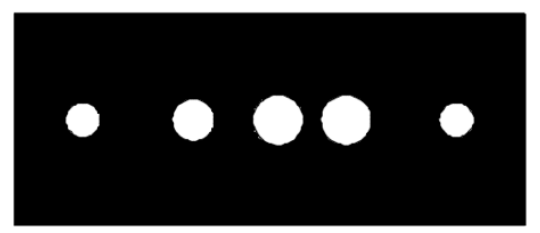

Layer one

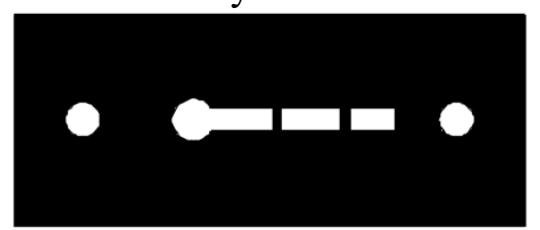

Layer three

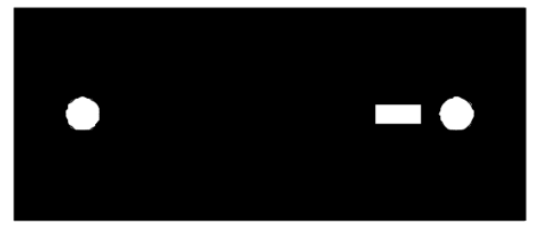

Layer four

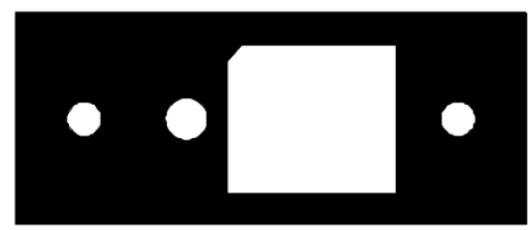

Layer two

Layer two (nitrocellulose)

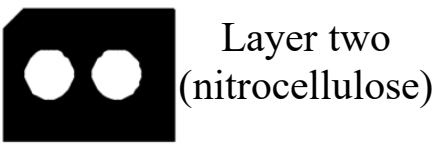

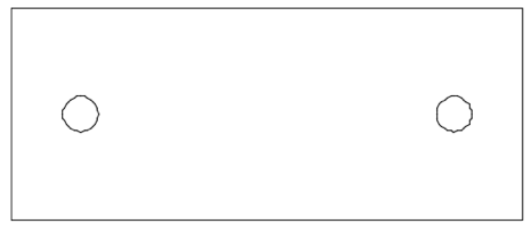

Layer five

Figure 18. Final design for one sample fluid flow validation test

This design follows a similar layout to the rotational design, where the sample will be placed on the second from the left hole on layer one and proceed to flow through the rest of the device, down to the second layer, across layer three, up to the second layer detection pad, down again to the third layer, up to the second layer control pad, and down through the fluid routing fourth layer to the absorbent pad. The design also went through a variety of phases during testing, including varying the distance of the hydrophobic gap that forces the fluid upward to the detection and control pads.

Later, a housing was built specifically for this test in order to improve connectivity between layers and more accurate mimic the intended final device functionality. The design was cut out of acrylic sheeting using a drill press. This housing can be seen in Figure 19 and the full dimensions can be found in the Appendix. This housing allowed for easier and faster assembly of the layers. The further right and left circles in each layer were cut out in order to be fastened together with a screw at each end. 

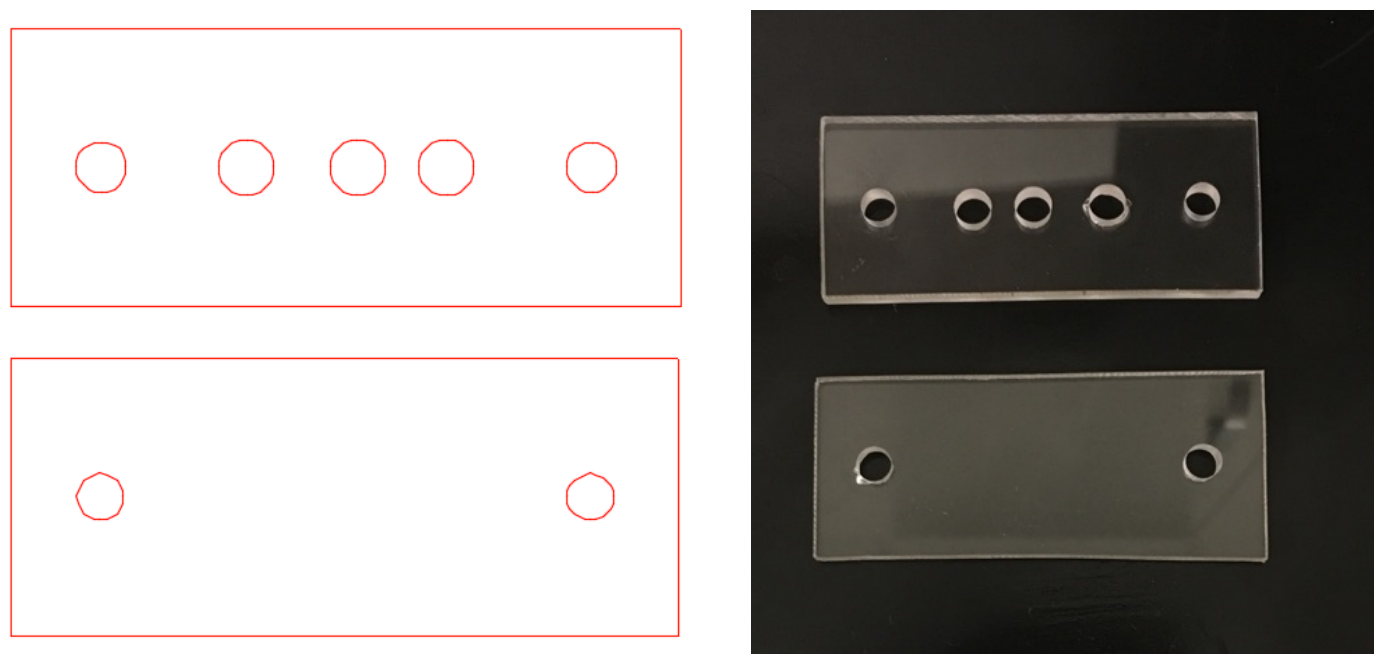

Figure 19. AutoCAD design and acrylic production of the one sample test flow housing

After the functional testing, the design was updated to include only a single print for the second layer, as opposed to two prints taped together as in previous designs. This began as a nitrocellulose "star" layer containing an area for the conjugate pad and a detection and control region which would be loaded with reagent and then taped to the underside of layer one, seen in Figure 20. This unit would then be rotated to expose the reagents to the sample and the subsequent wash steps. In the star layer - layer two - the inner circle would be punched out for alignment with the rest of the device. The next array of circles would be punched out as well, leaving an empty hole where the conjugate pad would be inserted. The conjugate pad would be correspondingly cut out of glass fiber pad using the same hole punch so that it would fit within the hole on the nitrocellulose star. Then, the second array of circles would be the detection region and the furthest out array of circles would be the control region. 

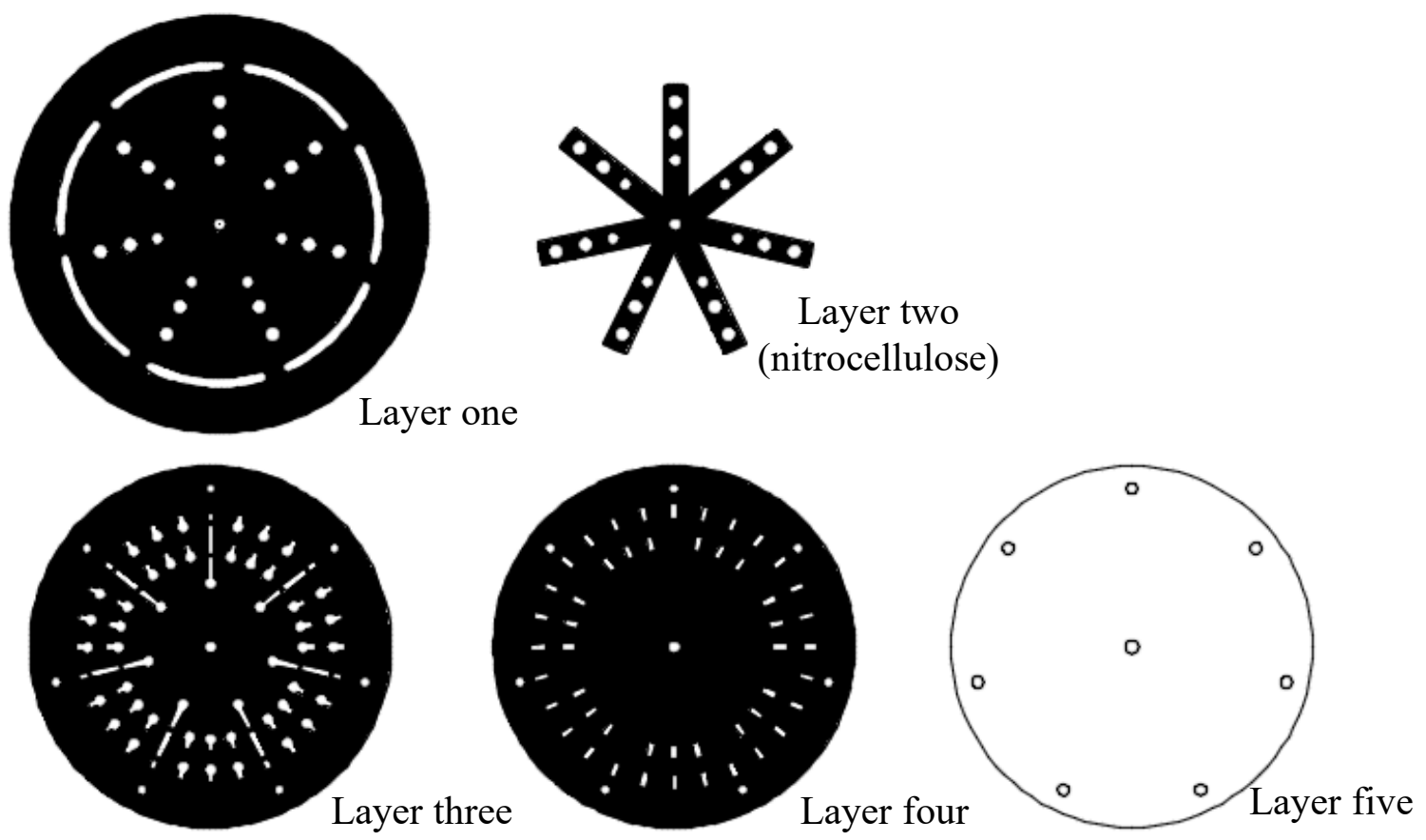

Figure 20. Nitrocellulose star detection layer design

This designed worked well for the initial tests where only two comparative samples were being run at once because each "limb" of the star could be cut off and placed on the back of the top cellulose layer. However, once the number of samples increased to all seven lanes, the nitrocellulose star became too fragile to work with and would rip in the center, making it more difficult to run a large number of tests at once. Therefore, the final design was changed to create a solid circle of wax-printed nitrocellulose for the second layer which was easier to handle and less prone to cracking and breaking. This design can be seen in Figure 21 and will be referred to as the final design. 

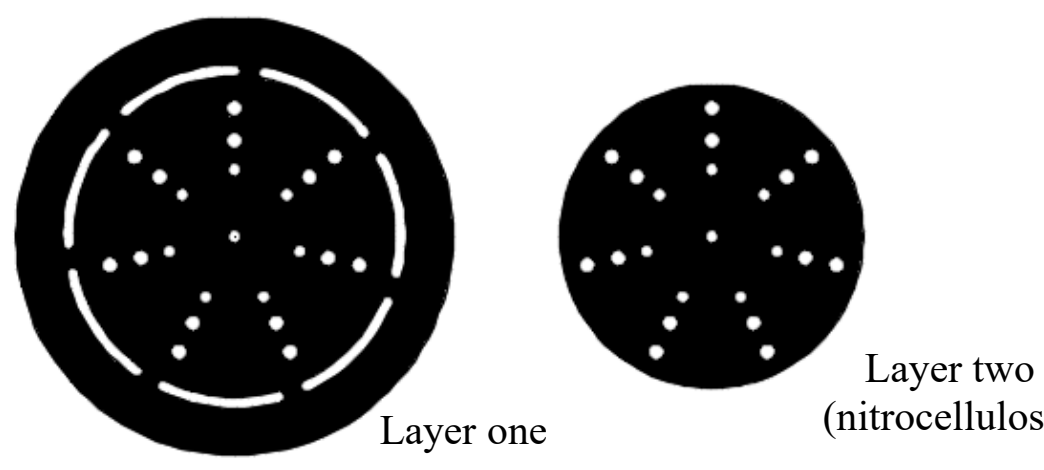

(nitrocellulose)
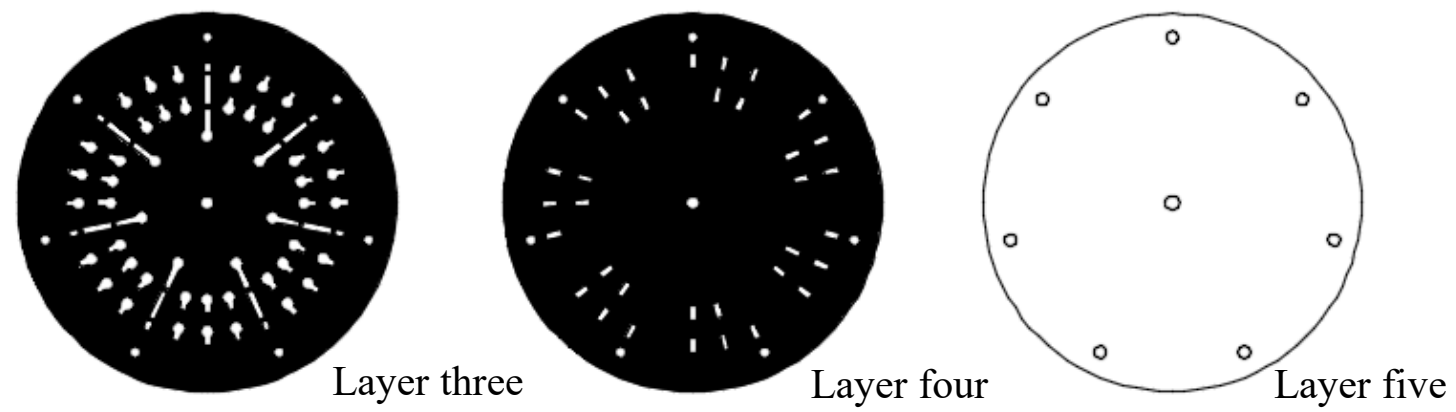

Figure 21. The final design for the paper-based $\mu$ PAD

This design does not include a fluid connectivity path to the bottom absorbent layer via the fourth layer for the final reagent wash step. This is due to the fact that the last reagent wash is intended to be a substrate for the enzyme on the detection pad and having it wash to the absorbent layer makes it difficult to accurately analyze the resultant chromogenic change. This is the design which will be used for the enzymatic assay tests. Before incorporation of the on-board reagents, however, the fluid flow through the device needed to be characterized.

\subsection{Dye Tests}

\subsubsection{Single Sample Device - Taped Assembly}

These tests were performed in order to determine the connectivity between the layers as well as form a baseline for the speed at which the sample would flow through the 
device. Since the optimization of channel widths and device timing tests had been qualified in previous theses under Dr. Clague, the timing of fluid flow was taken on a forinformation-only basis in order to make improvements to the device design in a go/no go determination [52] [53]. These tests were run with a solution made of a drop of red food dye in $15 \mathrm{~mL}$ of distilled water. All flow tests were performed using wax printed on cellulose paper which was subsequently melted on a hot plate for two minutes at $120^{\circ} \mathrm{C}$. After assembly of the layers, $30 \mu \mathrm{L}$ of the red dye solution was pipetted onto the sample pad and the stopwatch on an iPhone $6 \mathrm{~S}$ was started. Three lap times were taken: 1) when the red dye entered the detection pad, 2) when the red dye reached the control pad, and 3) when the red dye reached the absorbent pad. The final time was taken as the time to completion of the device and was determined by lifting up the petri dish and viewing underneath to see when the red dye appeared on the absorbent pad.

The first dye flow tests were performed on the one sample test strips, seen in Figure 18 in the above section. At first, the tests were performed by adhering each layer of the device together onto a plastic surface using double sided tape, as seen in Figure 22 below. 

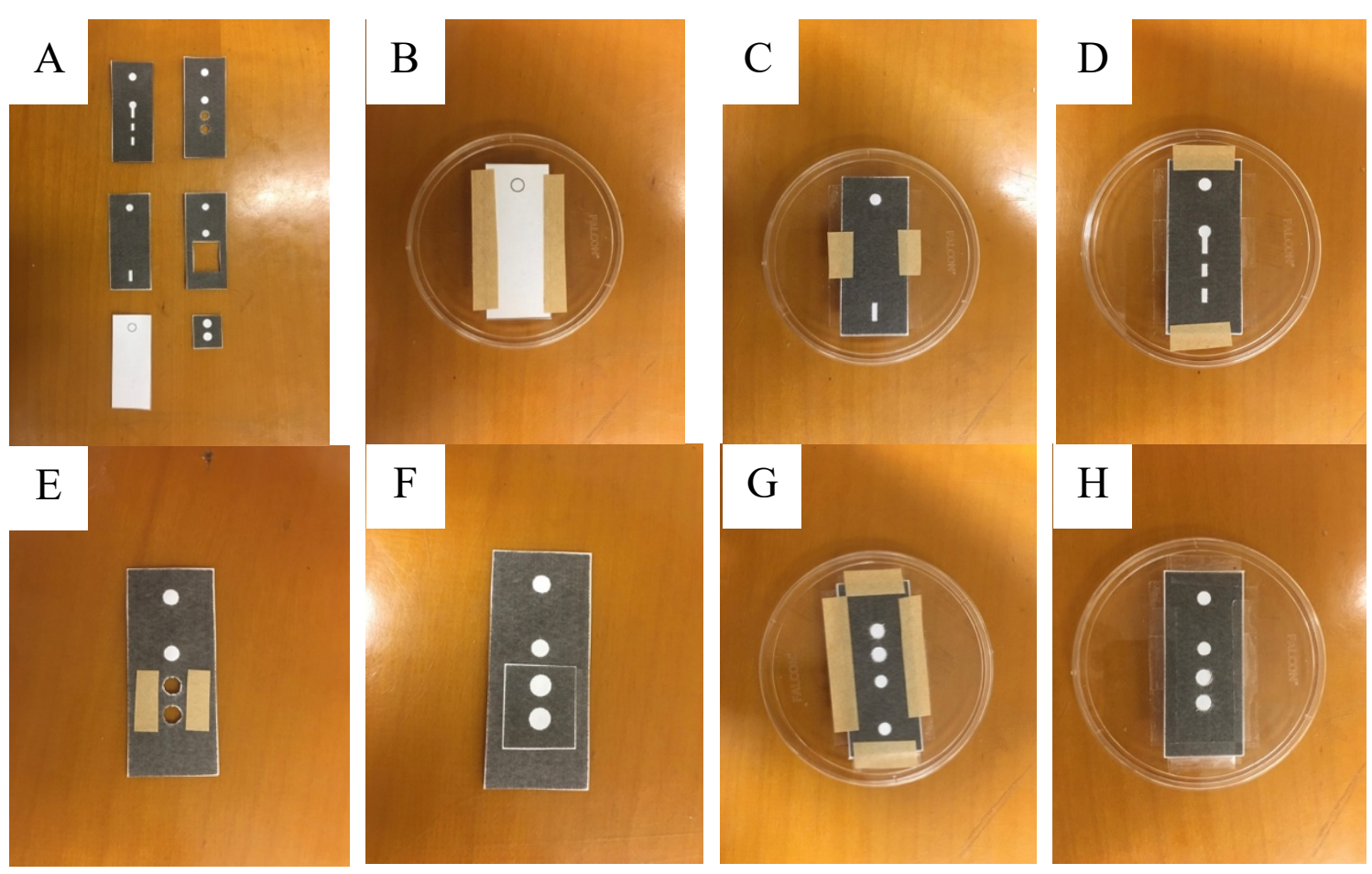

Figure 22. Process of taping device layers together to run validation tests, in order A-H

After wax melting was completed, the layers were cut out. Then, the absorbent pad (layer five) was taped down to the plastic petri dish. Following it was layers four and three, the fluid routing layers. Separately, the detection pad (layer two) was taped onto the underside of the sample pad (layer one). That assembly was then taped onto the top of layer three and the entire device was taped down to the petri dish again to ensure connectivity between layers in lieu of a housing.

\subsubsection{Single Sample Device - Housing Assembly}

After the initial dye routing tests, the design of the one sample flow was stable, and a housing was made which allowed for increased connectivity between layers and provided a sample port and open areas to add solution to the detection and control pads. For these tests, the design was printed onto cellulose with wax, melted for two minutes at $120^{\circ} \mathrm{C}$, and 
cut out, as before. Now, the distal holes for the screws were cut out of each layer as well. The device assembly and completed device can be seen below in Figure 23.

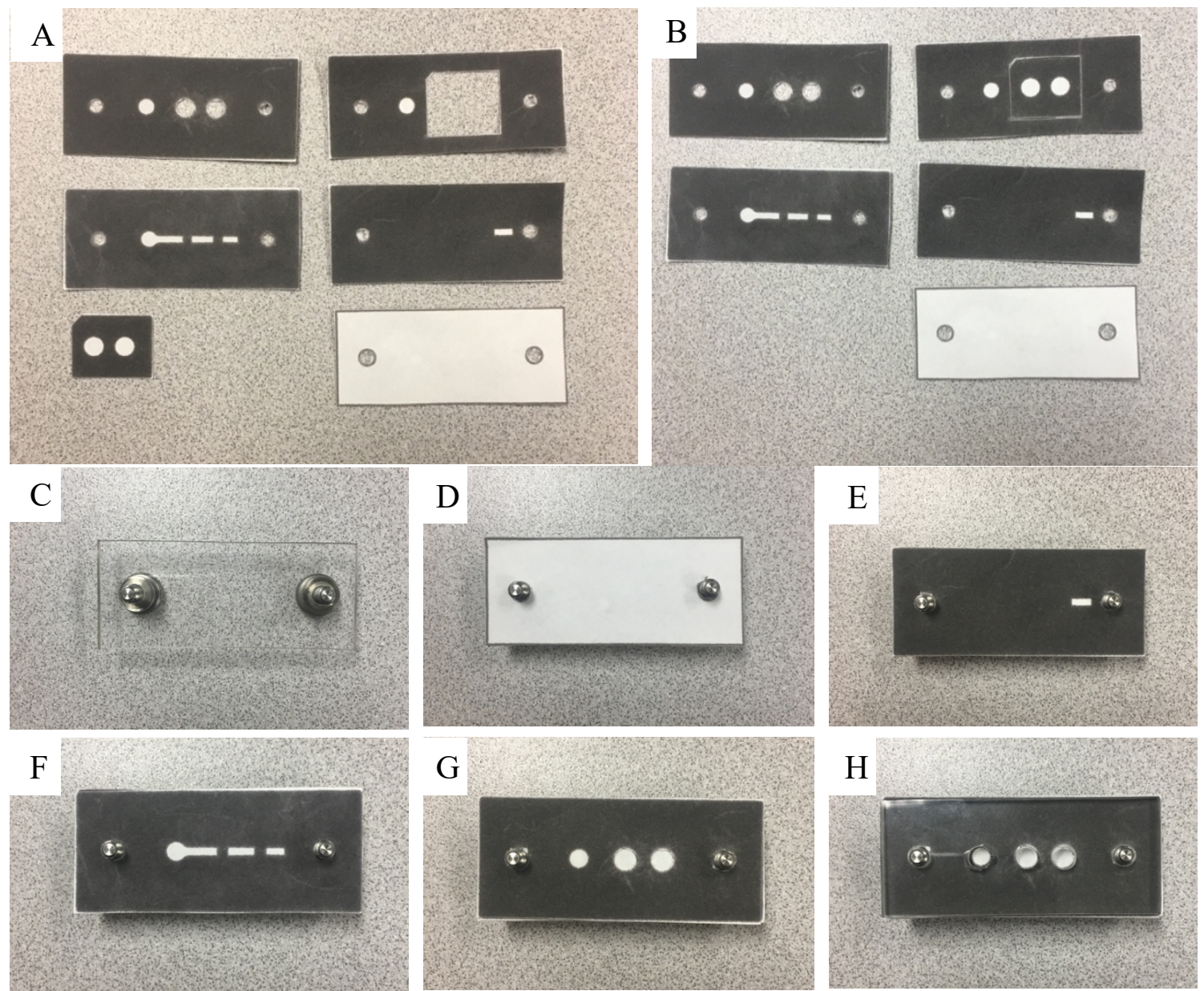

Figure 23. Process of assembling the device layers in the housing for the one sample fluid flow test, in order A-H

The detection layer was taped into the cut out of layer two, seen in the first images. Layer one had the detection and control areas cut out in order to be able to see the detection pad areas below. Then, as before, the device was assembled bottom-up starting with the bottom of the housing with both screws inserted as guidance for alignment. Continuing by placing in layer five, four, three, two, and one, before adding the top of the housing with the holes cut out. Hex nuts were attached onto the ends of the screws and to hold the housing together and secure the paper layers. 


\subsubsection{Rotating Assembly}

After the fluid flow within the single channel device had been completed, the final dimensions were translated into a rotational design with seven channels in a circular array. The star detection layer design, seen in Figure 20 in the previous section and in Figure 24 below, was tested using the red dye as before.

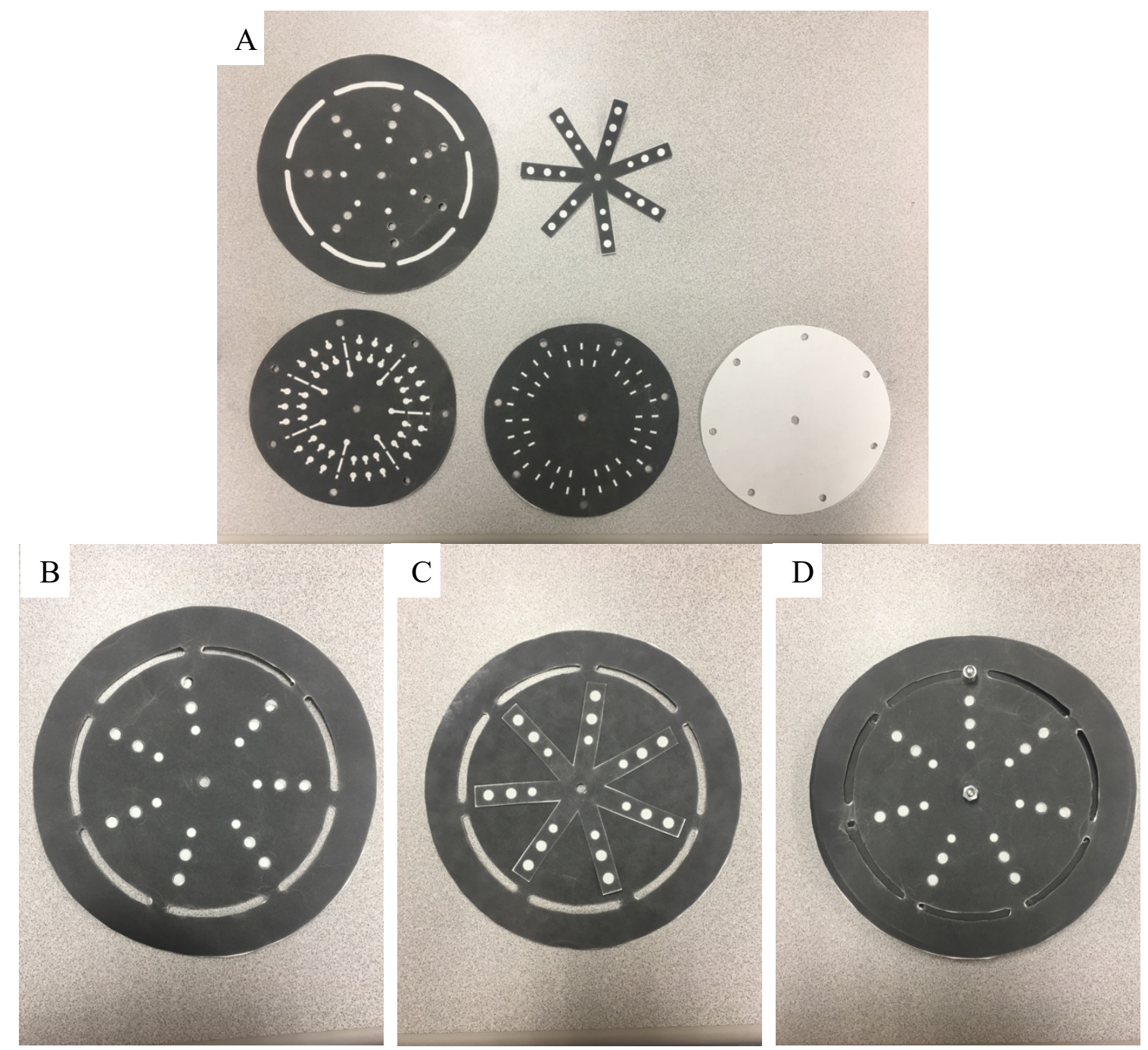

Figure 24. Nitrocellulose star design wax printed on cellulose and assembled, in order A-G

Again, the pieces were cut out and assembled bottom up, with the star layer taped to the bottom of layer one. The device was assembled without housing and held together with the screws at all points within a mocked-up plastic housing to allow for rotation. After this 
point, the device was determined to be fully functional as far as fluid flow was concerned and there was a low risk of wasting reagents due to an incorrectly designed, non-functional device.

\subsection{Housing Development}

The components of the housing were held together using two methods. At first, the housing pieces were held together using stainless steel raised knurled-head thumb screws (91830A206) from McMaster-Carr ${ }^{\circledR}$. This assembly is seen in Figure 25 below.

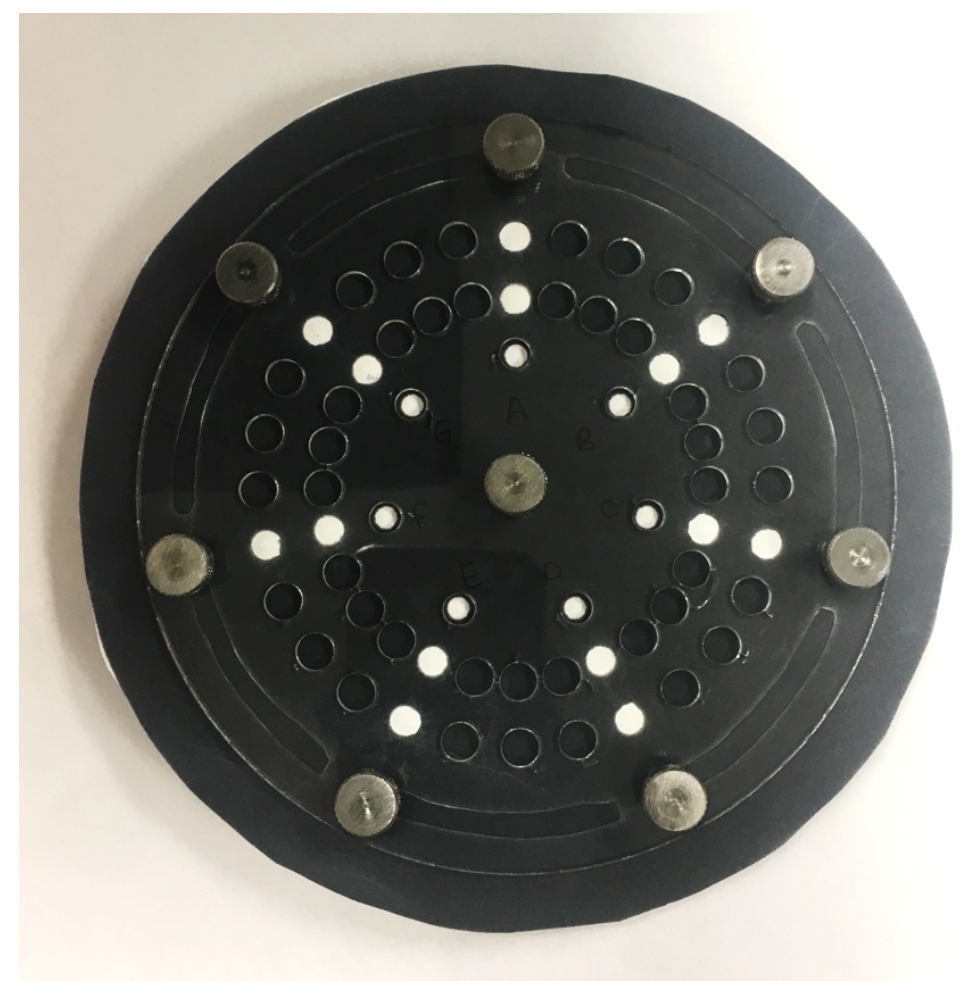

Figure 25. Housing assembly with knurled-head screws

As the experiments were conducted, it became apparent that the device would assemble easier if the layers could be held in proper alignment while the housing pieces are attached. The screws would have to be inserted while the user held the device in place, resulting in increased risk for user error. To mitigate against this, alloy steel cup-point set 
screw, 8-32 thread, 1-1/4" long (91375A176) were purchased from McMaster-Carr®. These screws could be inserted into the hex nut in the housing, as seen in Figure 26A below, allowing for precise assembly of the paper layers over the top of the screws. Then, the housing top was placed over the paper layers and hex nuts were screwed on the set screws to hold the housing together, as seen in Figure 26B.
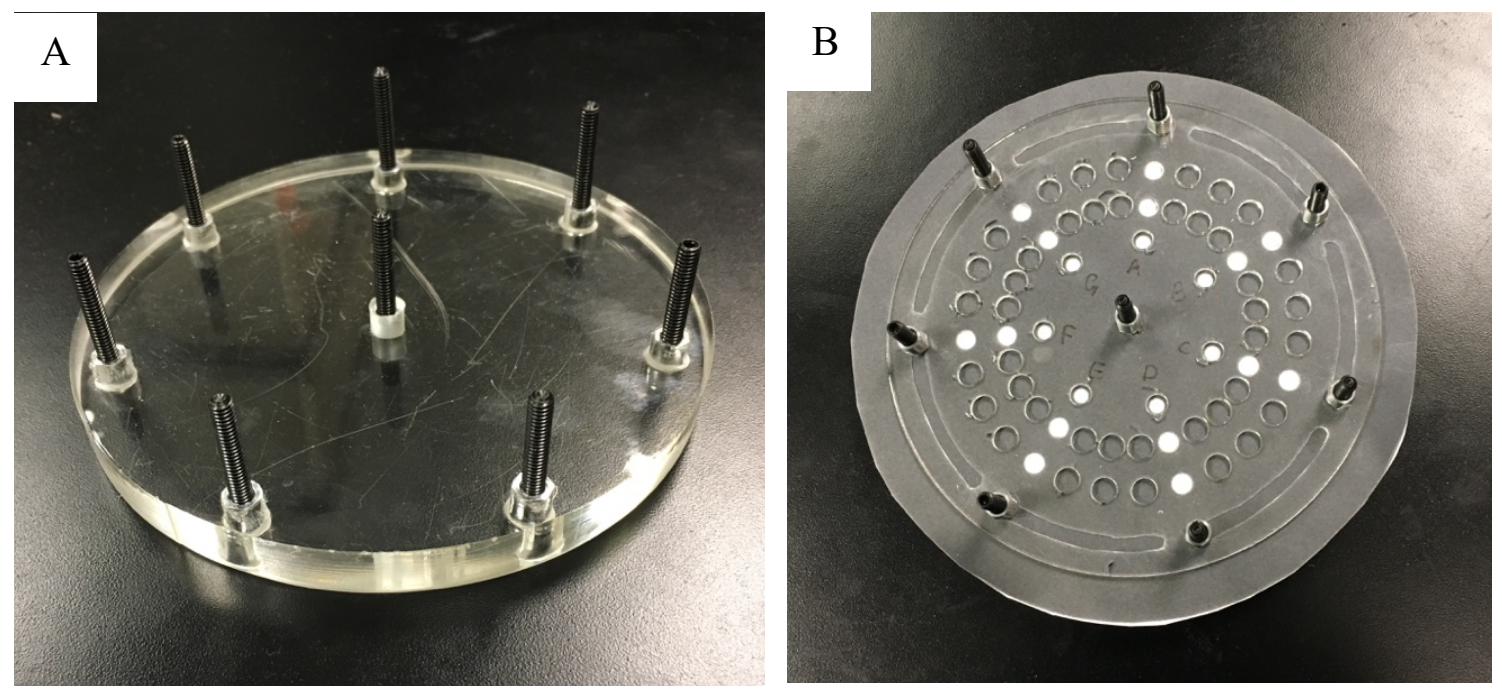

Figure 26. Housing with set screws, A) before device assembly and B) after device assembly

\subsection{Enzyme Assay Development}

After the device was fully characterized in regard to layer connectivity and fluid flow, the next step was to design a protocol which would allow for on-board reagents that would create the enzymatic assay. The design of the assay was based on the enzyme-linked immunosorbent assay (ELISA) procedure.

\subsubsection{Assay Preparation Procedure}

The assay antigen and stroke biomarker, recombinant human S100B protein (LSG26341) was purchased from LifeSpan Biosciences, Inc ${ }^{\circledR}$. Mouse monoclonal anti-S100B HRP-conjugated antibodies (ab10203) were purchased from abcam ${ }^{\circledR}$ to act as a detection 
antibody. Mouse polyclonal anti-S100B biotin-conjugated antibodies (ab48653) were also purchased from abcam ${ }^{\circledR}$ to act as a capture antibody. Rat anti-Mouse IgG1 biotinconjugated antibody (\#04-6140) was purchased from Thermo Fisher Scientific ${ }^{\circledR}$ as the control capture antibody against the detection antibody. Recombinant, mutant, nitrocellulose-binding streptavidin (Streptavidin-NC, PRO-338) was purchased from ProSpec ${ }^{\circledR}$ to aid in binding the capture antibodies to the nitrocellulose detection pad. TMB ELISA Substrate (Fast Kinetic Rate) (ab171524) was purchased from abcam ${ }^{\circledR}$ to product a chromogenic change in the assay when in contact with the HRP-conjugated detection antibody. Finally, bovine serum albumin (A9418-5G) was purchased from Millipore Sigma ${ }^{\circledR}$ to block the membranes and Phosphate-Buffered Saline (10010023) was purchased from Thermo Fisher Scientific ${ }^{\circledR}$ as a general laboratory reagent for diluting reagents and washing the assay.

The antigen and the streptavidin were diluted in PBS to a concentration of 0.1 $\mathrm{mg} / \mathrm{ml}$. The BSA was dissolved in PBS to a working concentration of $1 \% \mathrm{w} / \mathrm{v}(10 \mathrm{mg} / \mathrm{ml})$. Then, all antibodies were each diluted to a concentration of $0.1 \mathrm{mg} / \mathrm{ml}$ with the PBS $+1 \%$ BSA.

\subsubsection{Assay Implementation Procedure}

In general, all of the assays followed the same basic procedure in order to maintain consistency between data sets. Adjustments were made for each experiment and are detailed in the specific experimental section.

Printing:

1. Print every layer but the detection layer onto cellulose paper. 
2. Print the detection layer onto nitrocellulose paper using method described in previous section.

3. Melt the wax down on a hot plate at $120^{\circ} \mathrm{C}$ for 2 minutes for each layer.

4. Cut out applicable sections from each layer.

5. Hole punch a section of glass fiber pad for each conjugate pad, as seen in Figure 27 below (seven circles used for a full device).

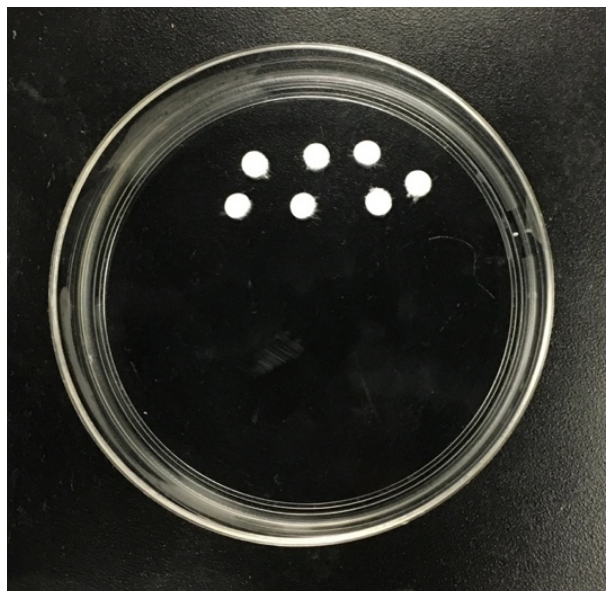

Figure 27. Hole-punched glass fiber for use as conjugate pads

Creation of Conjugate Pad - Detection Antibody:

1. Block each glass fiber pad (conjugate pad) by coating with $30 \mu 1$ of $1 \%$ BSA, as seen in Figure 28. Let sit for 30 minutes at room temperature.

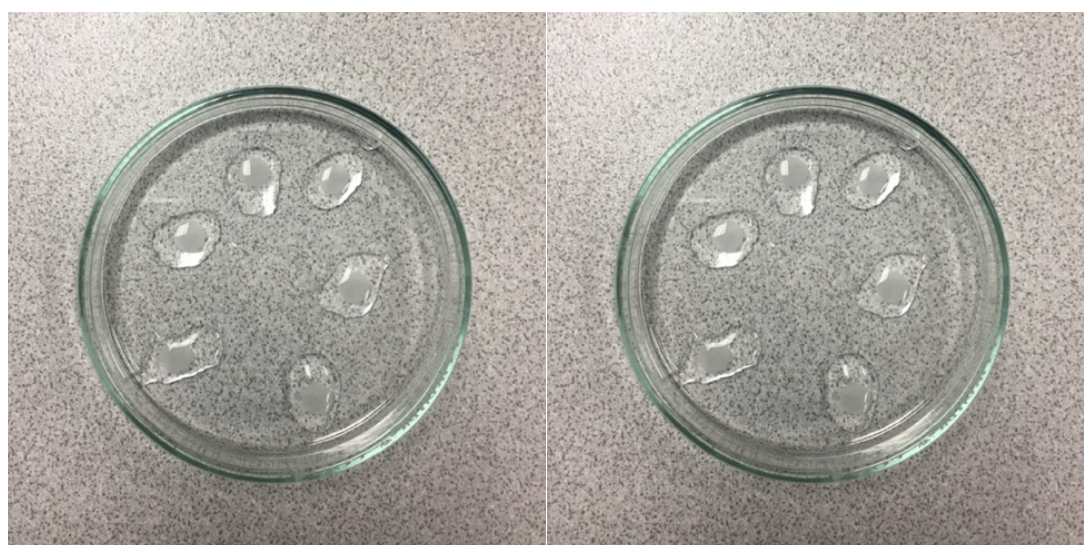

Figure 28. Glass fiber pads blocked with BSA 
2. Wash three times with $50 \mu \mathrm{l}$ PBS by pipetting PBS onto each pad and tilting the dish to collect reagent at the bottom to be collected by pipette and disposed of in liquid waste. Dry at $37^{\circ} \mathrm{C}$ for 45 minutes.

3. To each blocked glass fiber pad, add $10 \mu \mathrm{l}$ of $0.05 \mathrm{mg} / \mathrm{ml}$ HRP-conjugated detection antibody in $10 \%$ sucrose and $1 \%$ BSA. Dry at $37^{\circ} \mathrm{C}$ for 30 minutes.

Creation of Capture Pad - Capture Antibody:

1. Coat exposed nitrocellulose circles on nitrocellulose pad, Figure 29, with $5 \mu 1$ of the $0.1 \mathrm{mg} / \mathrm{ml}$ streptavidin solution. Dry at $37^{\circ} \mathrm{C}$ for 30 minutes.

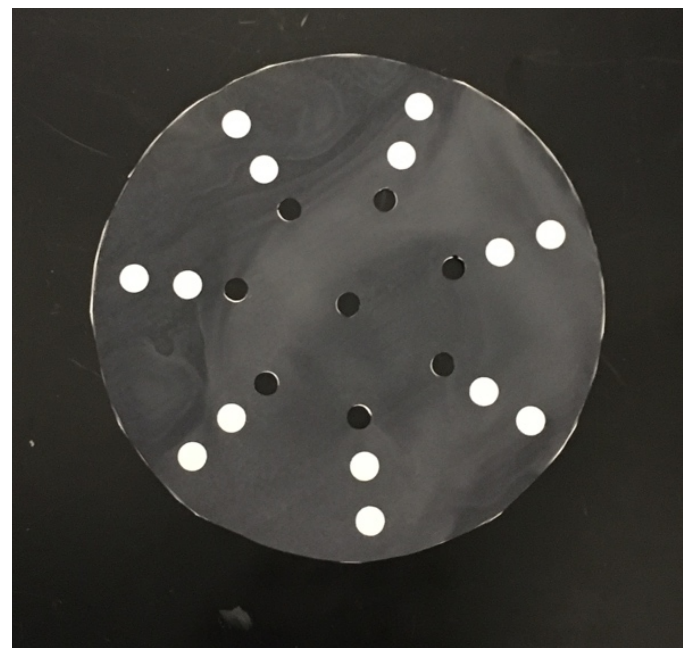

Figure 29. Nitrocellulose pad with specified holes cut out

2. Add $10 \mu \mathrm{l}$ of $0.1 \mathrm{mg} / \mathrm{ml}$ biotinylated anti-S100B antibody to detection pad region (first array of nitrocellulose circles). Add $10 \mu \mathrm{lof} 0.1 \mathrm{mg} / \mathrm{ml}$ biotinylated rat antimouse IgG1 antibody to control pad regions (second array of nitrocellulose circles). Dry at $37^{\circ} \mathrm{C}$ for 30 minutes.

3. Block the nitrocellulose with $10 \mu 1$ of $1 \%$ BSA solution. Let sit for 15 minutes.

4. Wash the detection and control pads three times with $20 \mu \mathrm{PBS}$. dry at $37^{\circ} \mathrm{C}$ for 30 minutes. 
Assay Demonstration:

1. Tape conjugate pads to nitrocellulose pad, as seen in Figure 30, by aligning the hole punched glass fiber circles to the hole punched region on the nitrocellulose and adhering using double-sided tape.
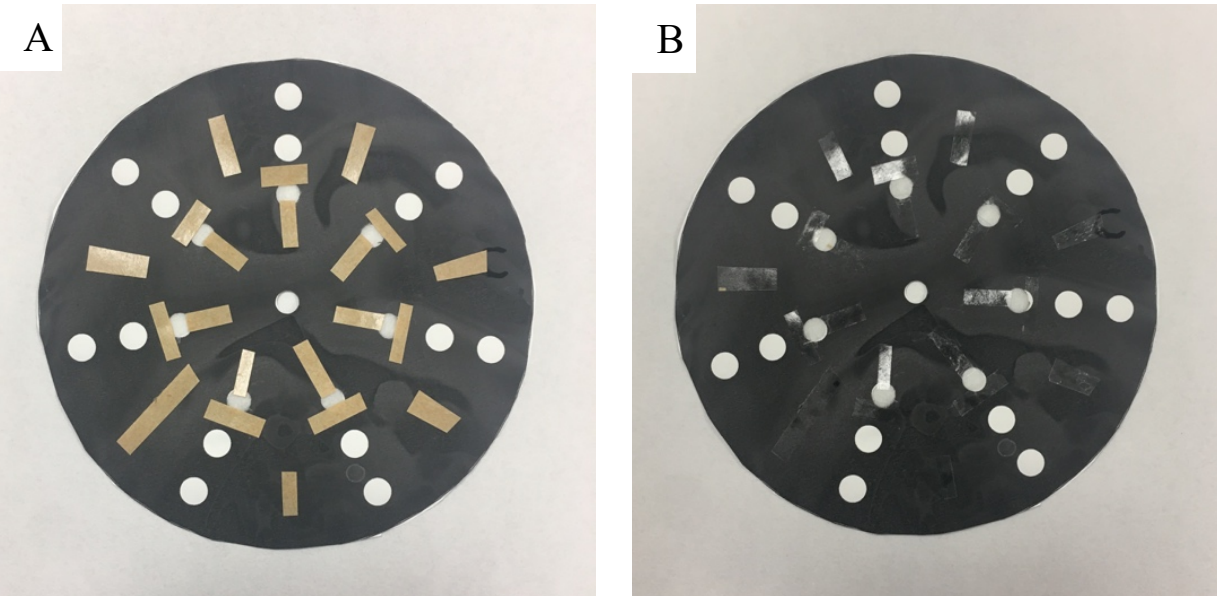

Figure 30. The process of attaching conjugate pads to nitrocellulose with double-sided tape, A) with tape backing attached and $B$ ) after tape backing is removed

2. Tape nitrocellulose layer to the bottom of layer one, aligning the holes in layer one to the detection and control pads on the nitrocellulose layer, Figure 31 below.
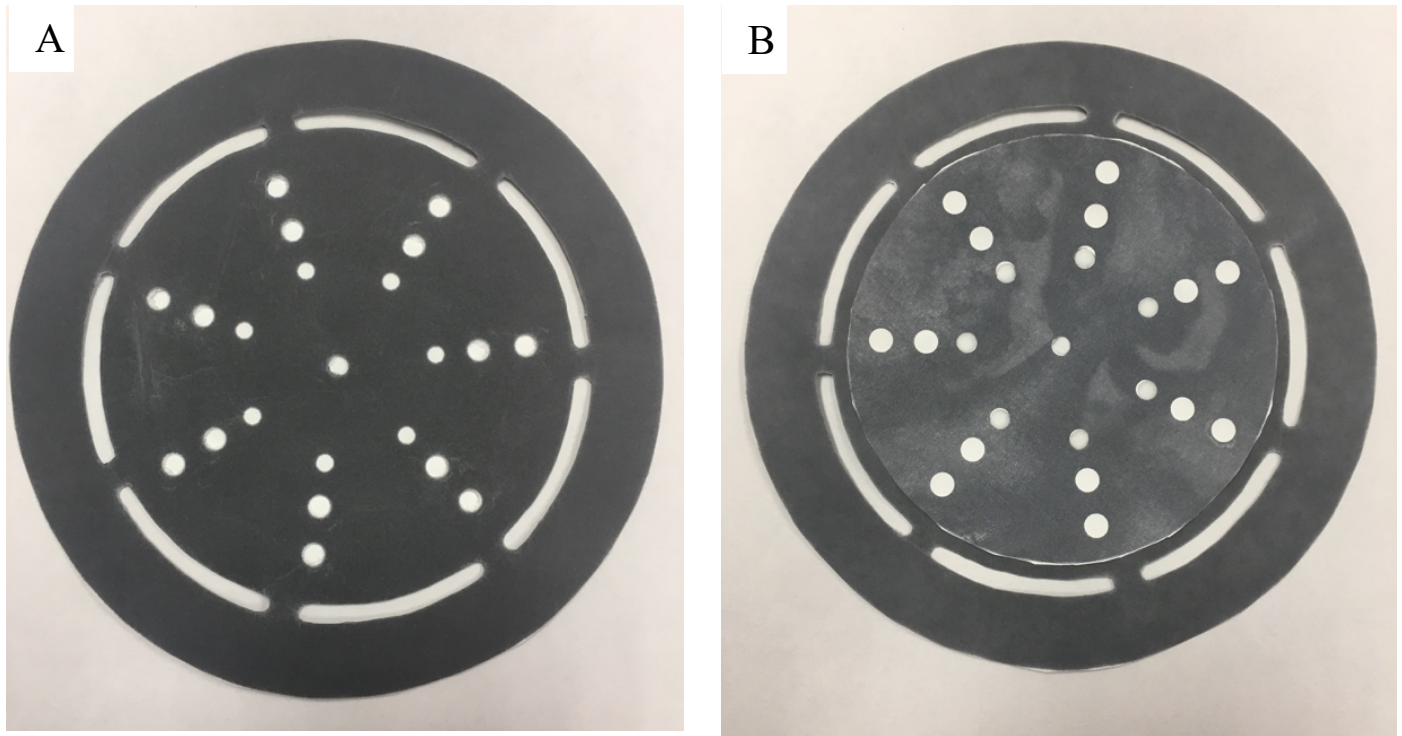

Figure 31. Front (A) and back (B) of layer one taped to the detection nitrocellulose pad 
3. Assemble device, layer by layer, as described previously, within the housing. The final assembled device can be seen in Figure 32.

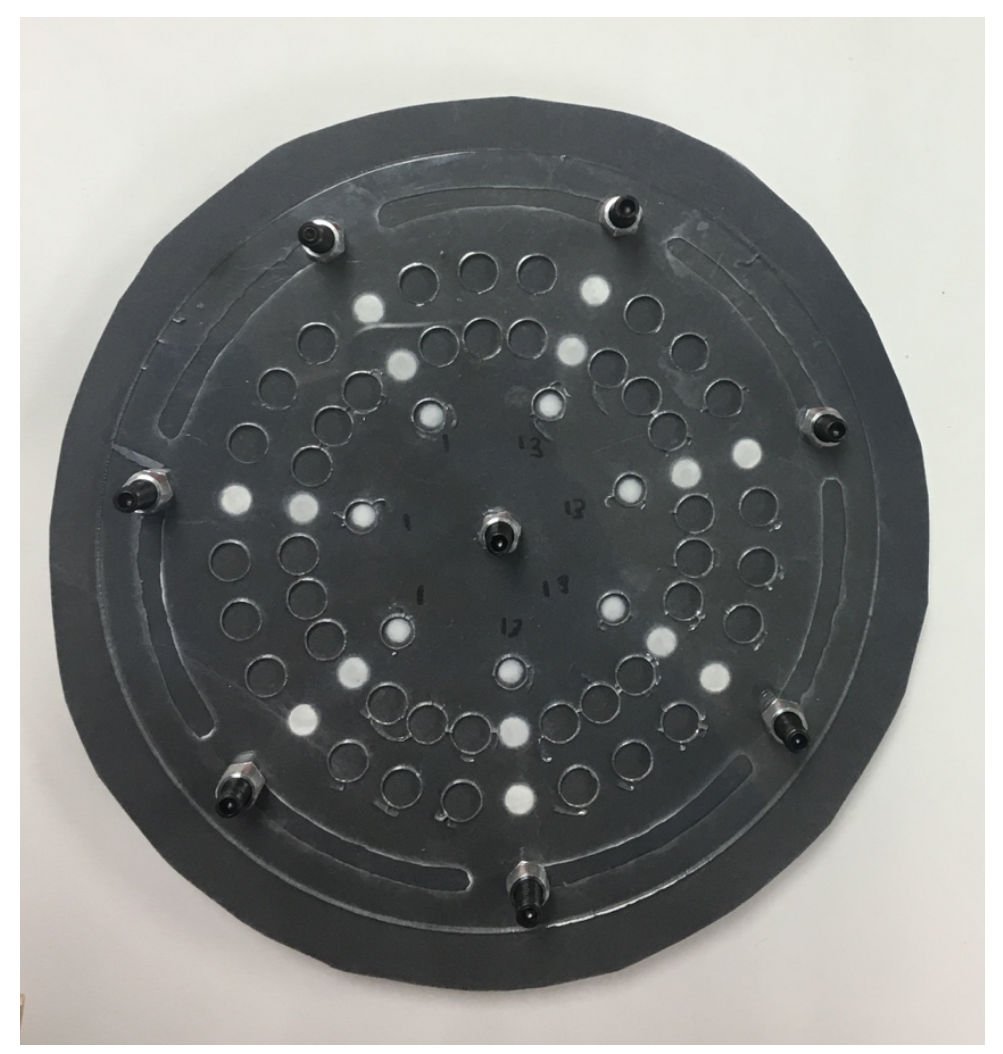

Figure 32. Fully assembled assay device prior to sample loading

4. Run $30 \mu 1$ of S100B sample of specified concentration. Wait for all sample to be wicked through the device so none remains on the sample pad.

5. Rotate, aligning the detection and control pads with the holes in the housing, as seen in Figure 33, and wash each pad with $30 \mu 1$ PBS. Wait for all PBS to be wicked through to the absorbent layer. 


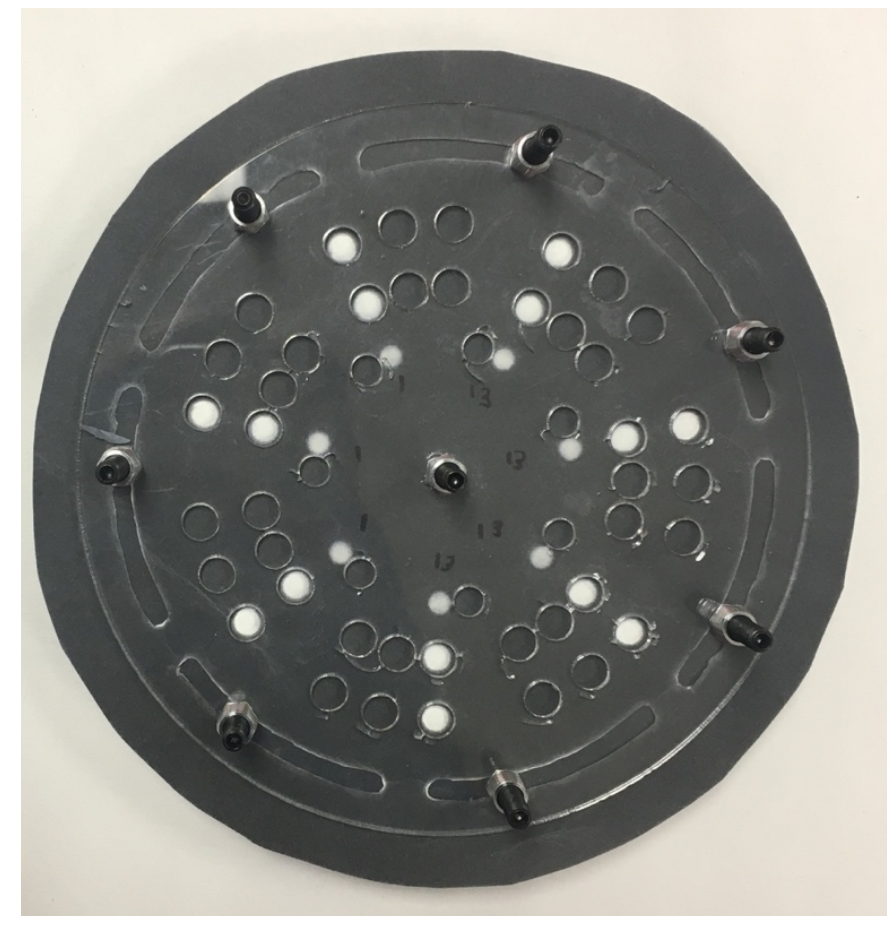

Figure 33. Device after first rotation and first PBS application

6. Rotate again, Figure 34, and wash each pad with $30 \mu 1$ PBS. Wait for all PBS to be wicked through to the absorbent layer.

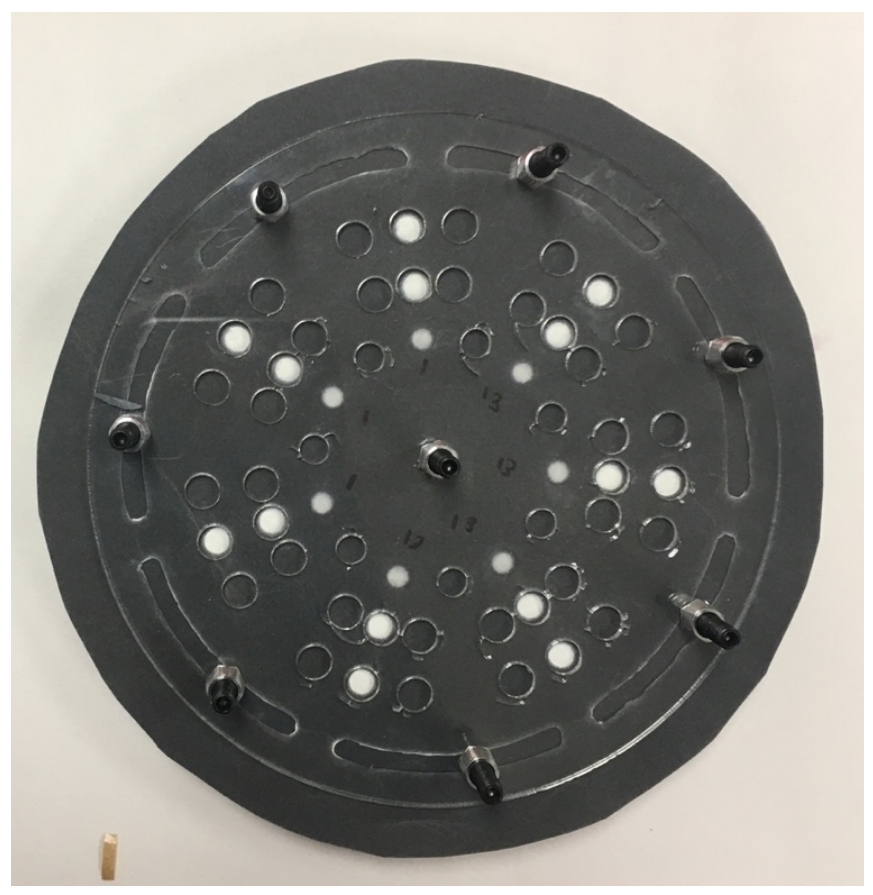

Figure 34. Device after second rotation and second PBS application 
7. Rotate again, aligning to the last circles, and add $10 \mu \mathrm{TMB}$ to each detection pad.

8. Quickly, take a clear photo of the device from above.

\subsubsection{Image Analysis}

Two forms of image analysis were used in order to quantify the intensities of the color appearing on each detection pad. First, the MATLAB code created by Van Huynh (2016) was utilized, following the user instructions prompted by the code in order to return average intensity values with arbitrary units [58]. ImageJ was used once it became clear that the MATLAB code did not provide the level of specificity required for the assay. The ImageJ software was used in conjunction with a downloaded plugin called "RBG Measure" [59]. This plugin was used to calculate the average red, blue, and green values within each detection region of an image in order to better quantify the control region as background. For this form of analysis, the background was removed with the ImageJ rolling ball setting at 50 pixels, the image was inverted, and the red, green, and blue (RGB) values were measured for each region of interest, i.e. the detection pads. Then, the R, G, and B values were averaged for each region of interest to obtain an average color intensity for each sample and the control. Finally, the average color intensity of the sample was divided by that of the control in order to obtain a signal-to-noise ratio.

\subsubsection{Antibody Analysis}

The first assay experiment was completed using the nitrocellulose star design, seen in Figure 20 above. The purpose of this experiment was to determine if the purchase antibodies and antigen interacted properly with each other and could develop a blue color 
signal. The device was run with $10 \mu 1$ of $0.1 \mathrm{mg} / \mathrm{ml}$ HRP conjugated antibody in PBS + $10 \%$ sucrose and $0.1 \% \mathrm{BSA}, 10 \mu \mathrm{l}$ of $0.1 \mathrm{mg} / \mathrm{ml}$ biotinylated anti-S100B antibody, and 30 $\mu 1$ of $0.1 \mathrm{mg} / \mathrm{ml} \mathrm{S100B}$ antigen. The assay was otherwise run as described in the Assay Implementation Procedure section.

\subsubsection{Streptavidin Characterization}

A side experiment was conducted to investigate the use of streptavidin + biotin on the detection pad. As mentioned, the streptavidin purchased for this thesis was a mutant, nitrocellulose-binding streptavidin protein. This protein was purchased with the thought that it would bind better to the detection pad and therefore binding to more of the biotinylated capture antibody and preventing the reagents washing off with the sample fluid front or PBS washes. The efficacy of this protein was investigated by comparing it to native streptavidin (ab136200) from abcam ${ }^{\circledR}$ and to a control detection pad without any streptavidin. On three detection pads, $5 \mu 1$ of $0.1 \mathrm{mg} / \mathrm{ml}$ mutant streptavidin was deposited. On another three detection pads, $5 \mu 1$ of $0.1 \mathrm{mg} / \mathrm{ml}$ native streptavidin was deposited. The seventh detection pad was treated with $5 \mu 1$ of PBS as a control. Then, as in the protocol, the biotinylated anti-S100B antibody was deposited and dried, followed by a BSA blocking step. Then, after device assembly, $30 \mu 1$ of $1 \mathrm{ng} / \mathrm{ml} \mathrm{S100B}$ protein was added to each sample pad. The rest of the experiment was run as in the protocol.

\subsubsection{Optimization of Detection Antibody}

The second major experiment conducted was to determine the effect that the concentration of HRP-conjugated anti-S100B antibody has on the sensitivity of the assay 
on this particular device. To optimize this parameter, the concentration of detection antibody was varied between $0.01 \mathrm{mg} / \mathrm{ml}$ to $0.1 \mathrm{mg} / \mathrm{ml}$ with a blank included. The antibody was serially diluted in PBS $+1 \%$ BSA $+10 \%$ in order to return the following concentrations: $0.1 \mathrm{mg} / \mathrm{ml}, 0.08 \mathrm{mg} / \mathrm{ml}, 0.06 \mathrm{mg} / \mathrm{ml}, 0.04 \mathrm{mg} / \mathrm{ml}, 0.02 \mathrm{mg} / \mathrm{ml}, 0.01 \mathrm{mg} / \mathrm{ml}$, and a $0 \mathrm{mg} / \mathrm{ml}$ diluent blank. The assay was run in triplicate using $30 \mu \mathrm{l}$ of $0.1 \mu \mathrm{g} / \mathrm{ml} \mathrm{S100B}$ sample.

\subsubsection{Determination of Limit of Detection}

Then, the assay was tested with a panel of protein concentrations in order to determine the limit of detection (LOD), defined as the lowest analyte concentration which can be reliably differentiated from a blank [60]. The aim was to derive a standard curve, similar to that of an ELISA, which follows a sigmoidal shape when plotting analyte concentration versus the signal-to-noise ratio. To achieve this, a serial dilution of S100B was performed to cover a range of $0.1 \mathrm{mg} / \mathrm{ml}$ to $1 \mathrm{E}-4 \mathrm{pg} / \mathrm{ml}$ in order to get a complete range of responses.

\subsubsection{Analysis of Troponin-Based Assay}

The previous thesis completed by Holler (2016) was not able to display full device functionality with on-board reagents, specifically, in regard to the detection antibody loaded onto and resolubilized from the conjugate pad. In that work, the conjugate pad was made out of cellulose paper, as compared to the glass fiber used for the device in this thesis. In order to conduct an initial investigation into whether this pad material had an effect on the functionality of the device and the resolubilization of the detection antibody, the 
reagents used by Holler (2016) were incorporated into the device created for this thesis using the protocol established above. Since the previous work of Holler (2016) used troponin as the analyte, this investigation was also meant to establish ground work as to whether the device and protocol could be adapted to a protein other than S100B.

First, the anti-cardiac troponin I capture antibody (ab47003) was biotinylated using the Biotinylation Kit/Biotin Conjugation Kit (Fast, Type B) (ab201796) following the associated kit protocol. Following the streptavidin application, as in the protocol, $10 \mu 1$ of capture antibody was loaded onto the device, four detection pads at $1.6 \mathrm{mg} / \mathrm{ml}$ and three detection pads at $0.1 \mathrm{mg} / \mathrm{ml}$ in order to get a range for device detection ability, which had not been established. The HRP anti-cardiac troponin detection antibody (ab10239) was deposited onto the glass fiber conjugate pad, $10 \mu \mathrm{l}$ at $0.05 \mathrm{mg} / \mathrm{ml}$. The assay was assembled and run with $30 \mu \mathrm{l}$ of $1 \mathrm{ng} / \mathrm{ml}$ troponin protein (ab9936). 


\section{RESULTS}

This portion of the report will summarize the results from the experiments performed in order to fully validate the development of the paper-based $\mu$ PAD with ELISA-like technology integrated within the device.

\subsection{Analysis of Previous Work}

After running red dye through the sample port of Alicia Holler's previous design assembled within her housing, the device was taken apart to visualize the fluid flow pathway. This can be seen in Figure 35 below.

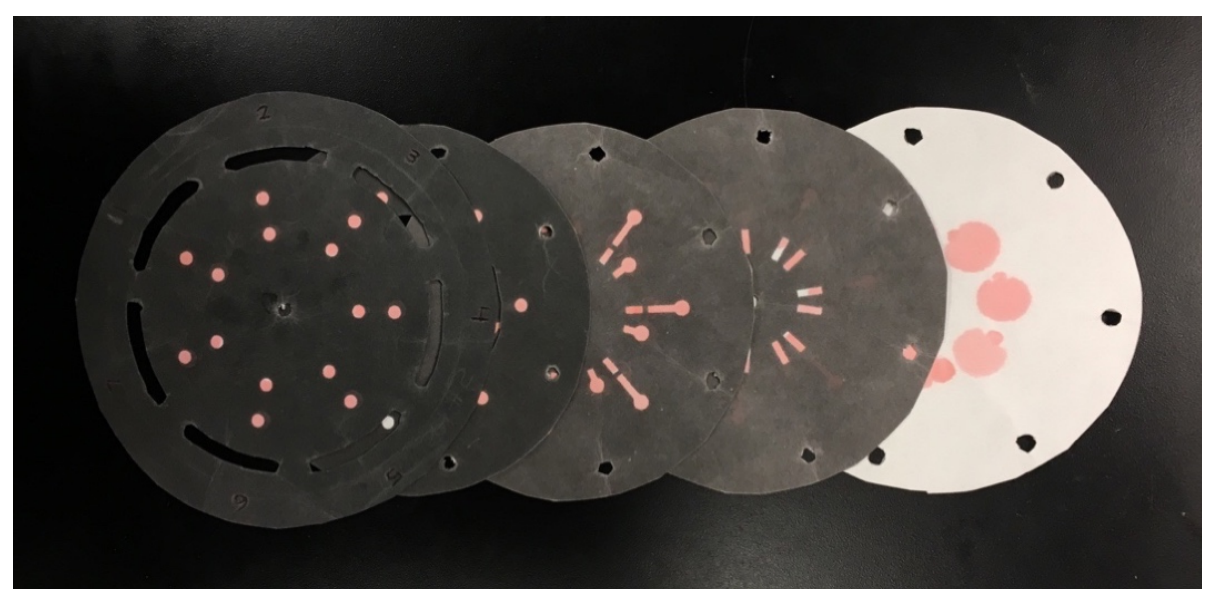

Figure 35. Fluid flow analysis of Holler's design with red dye

\subsection{Dye Tests}

\subsubsection{Single Sample Device - Taped Assembly}

The first characterization tests of the new design were completed on single-channel devices, with which the design could be quickly and efficiently changed to adapt to the results before being implemented into the rotational design. Several trials of this design were run where each layer was taped together; however, five trials out of the six trials did not see proper fluid routing, and, while care was taken to assemble the device as accurately 
as possible, the tape method was not substantial enough to provide fluid flow data. Most often, it was observed that the fluid front stopped after the detection pad before reaching the control pad. The reasoning behind this will be analyzed further in the Discussion.

The one complete trial can be seen in the figures below, in which the red dye has been placed on the sample pad, the second circle from the top on the device, and was able to flow through the entirety of the device, appearing on both the detection pad and the sample pad, the following circles, third and fourth from the top.
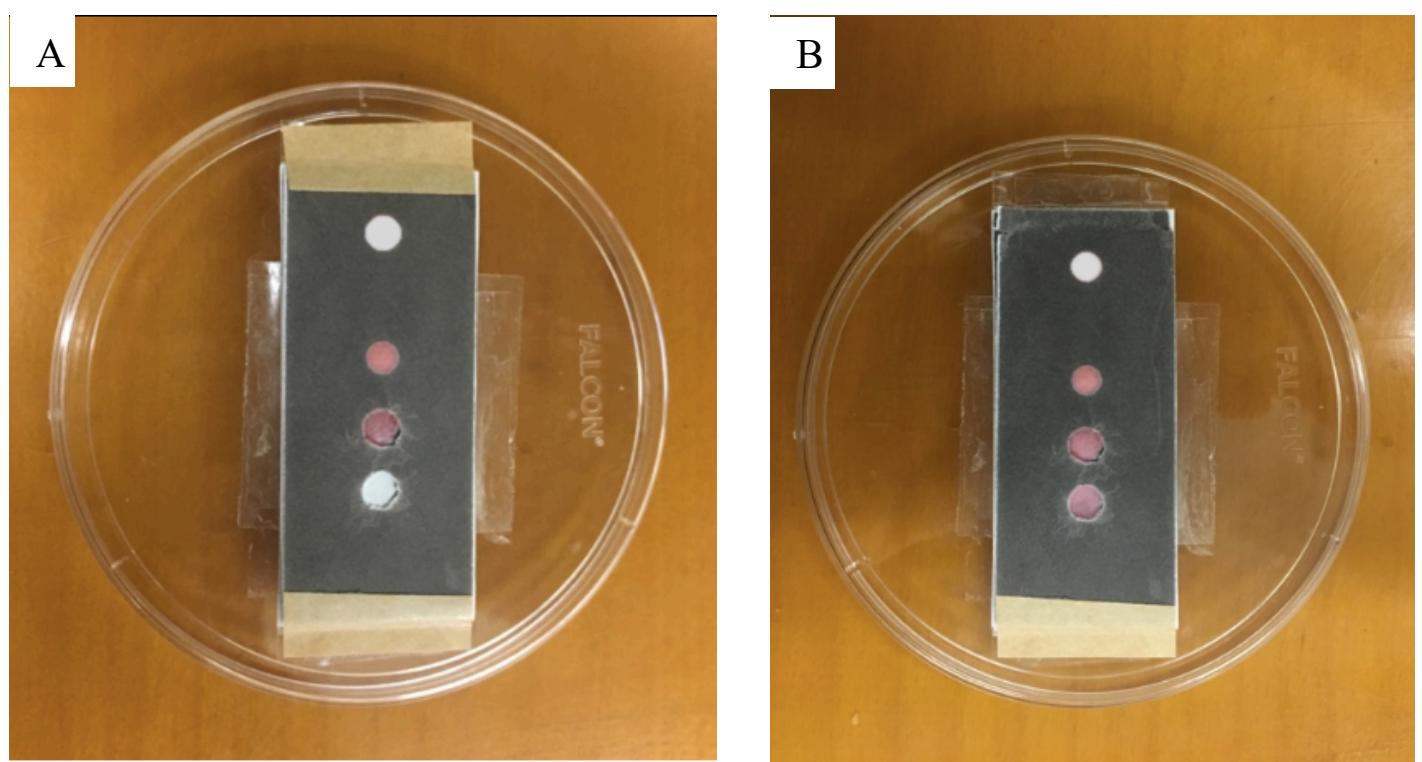

Figure 36. Progression of red dye through single sample device, A) at detection pad and B) at control pad region

The time was taken as described in the Methods. The elapsed time for the trial is summarized in Table II below.

Table II. Development Time for Single Sample Fluid Flow

\begin{tabular}{|c|c|c|c|}
\hline & $\begin{array}{c}\text { Time to Detection Pad } \\
\text { (seconds) }\end{array}$ & $\begin{array}{c}\text { Time to Control Pad } \\
\text { (seconds) }\end{array}$ & $\begin{array}{c}\text { Time to Absorbent Pad } \\
\text { (seconds) }\end{array}$ \\
\hline Trial 1 & $14.80 \pm 0.2$ & $45.51 \pm 0.2$ & $\begin{array}{c}138.54 \pm 0.2 \\
(2: 18.54 \text { minutes) }\end{array}$ \\
\hline
\end{tabular}




\subsubsection{Single Sample Device - Housing Assembly}

Once the design of the single sample device had been finalized, a housing was created and used to test further iterations to better approximate the fluid flow that would be expected in the final rotating device. The progression of fluid flow can be seen in Figure 37 below:
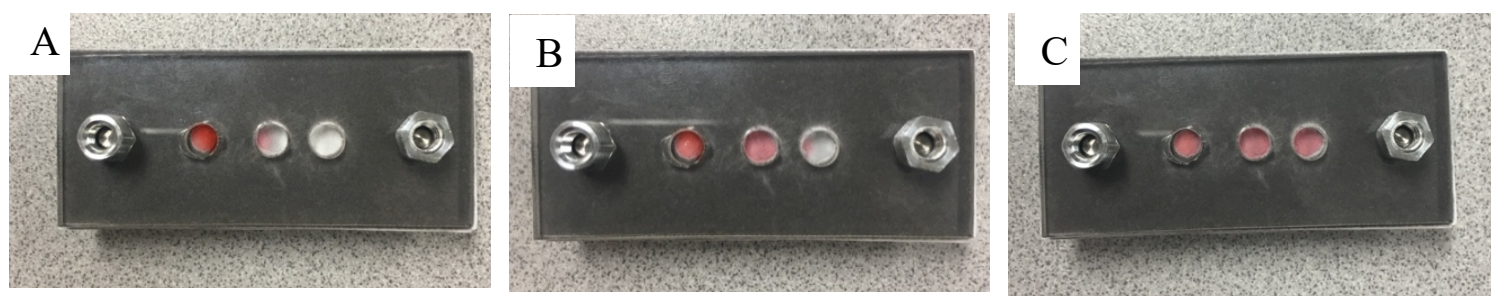

Figure 37. Flow of red dye through single sample device with housing, A-C as time elapses

When taken apart, one can see that the red dye was able to flow through the device via the channels, successfully reaching the absorbent pad within the housing, as seen in Figure 38 .
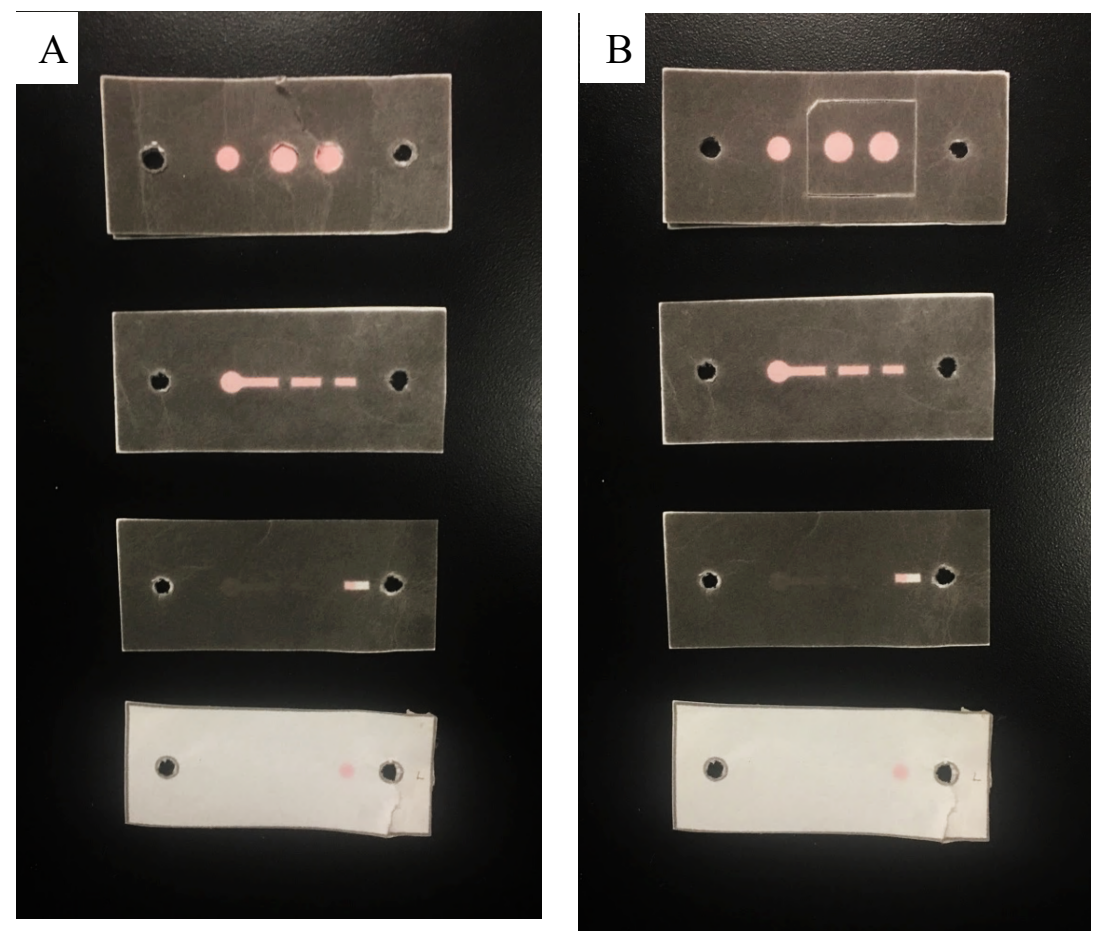

Figure 38. Results of fluid flow testing of single sample device; A) top of layer one and B) bottom of layer one showing the detection pad 
In all trials, the time was taken as described in the Methods. The elapsed time for each trial is summarized in Table III below.

Table III. Development Times for Single Sample Device with Housing

\begin{tabular}{|c|c|c|c|}
\hline & $\begin{array}{c}\text { Time to Detection Pad } \\
\text { (seconds) }\end{array}$ & $\begin{array}{c}\text { Time to Control Pad } \\
\text { (seconds) }\end{array}$ & $\begin{array}{c}\text { Time to Absorbent Pad } \\
\text { (seconds) }\end{array}$ \\
\hline Trial 1 & $17.75 \pm 0.2$ & $77.53 \pm 0.2$ & $\begin{array}{c}210.13 \pm 0.2 \\
(3: 30.13 \text { minutes) }\end{array}$ \\
\hline Trial 2 & $12.30 \pm 0.2$ & $46.71 \pm 0.2$ & $\begin{array}{c}139.25 \pm 0.2 \\
(2: 19.25 \text { minutes) }\end{array}$ \\
\hline Trial 3 & $14.65 \pm 0.2$ & $43.96 \pm 0.2$ & $\begin{array}{c}133.36 \pm 0.2 \\
(2: 13.36 \text { minutes })\end{array}$ \\
\hline
\end{tabular}

These results were averaged and the standard deviation of each was determined, as seen in Table IV below.

Table IV. Average Development Times for Single Sample Device with Housing

\begin{tabular}{|c|c|c|c|c|c|}
\hline $\begin{array}{l}\text { Average } \\
\text { Time to } \\
\text { Detection } \\
\text { Pad (s) }\end{array}$ & $\begin{array}{l}\text { Standard } \\
\text { Deviation } \\
\text { of Time to } \\
\text { Detection } \\
\text { Pad (s) }\end{array}$ & $\begin{array}{l}\text { Average } \\
\text { Time to } \\
\text { Control } \\
\text { Pad (s) }\end{array}$ & $\begin{array}{c}\text { Standard } \\
\text { Deviation } \\
\text { of Time to } \\
\text { Control } \\
\text { Pad (s) }\end{array}$ & $\begin{array}{c}\text { Average } \\
\text { Time to } \\
\text { Absorbent } \\
\text { Pad (s) }\end{array}$ & $\begin{array}{l}\text { Standard } \\
\text { Deviation of } \\
\text { Time to } \\
\text { Absorbent } \\
\text { Pad (s) }\end{array}$ \\
\hline $14.9 \pm 0.2$ & 2.73 & $56.07 \pm 0.2$ & 18.64 & $160.91 \pm 0.2$ & 42.72 \\
\hline
\end{tabular}

\subsubsection{Rotating Assembly}

The finalized dimensions from the single sample devices were then translated into a rotational design, as described in the Methods section, and included additional channels which could be used as wash steps. This design was also tested for fluid flow characteristics with red dye. The assembled device can be seen in Figure 39 below, both within the housing and disassembled to visualize the flow path. 

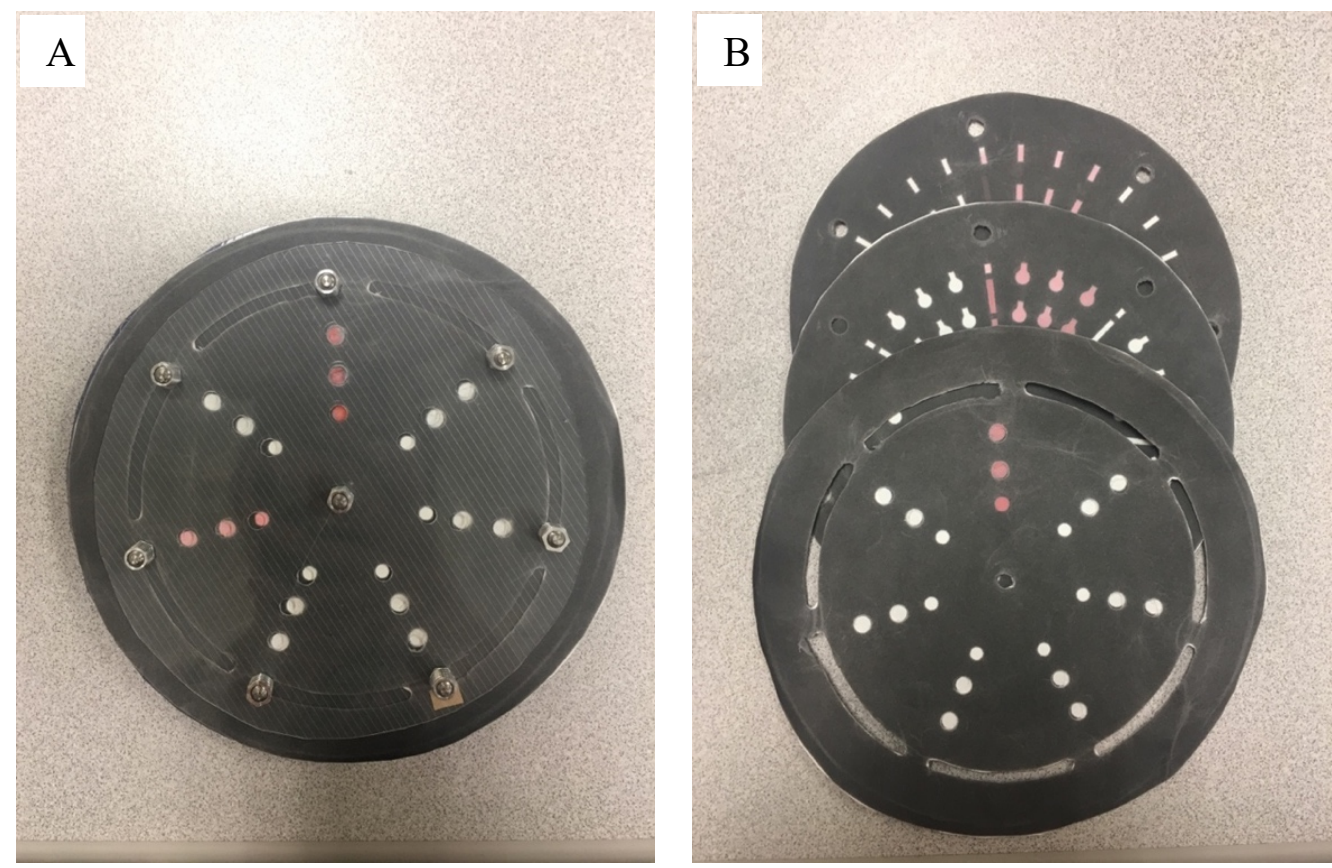

Figure 39. Results of rotational design for characterization of fluid flow, A) assembled and B) disassembled

As before, the time for the dye to flow through the device was taken for each trial and is displayed in Table $\mathrm{V}$ below.

Table V. Development Times for Rotational Device

\begin{tabular}{|c|c|c|c|}
\hline & $\begin{array}{c}\text { Time to Detection Pad } \\
\text { (seconds) }\end{array}$ & $\begin{array}{c}\text { Time to Control Pad } \\
\text { (seconds) }\end{array}$ & $\begin{array}{c}\text { Time to Absorbent Pad } \\
\text { (seconds) }\end{array}$ \\
\hline Trial 1 & $43.05 \pm 0.2$ & $84.46 \pm 0.2$ & $\begin{array}{c}123.56 \pm 0.2 \\
(2: 03.59 \text { minutes) }\end{array}$ \\
\hline Trial 2 & $29.13 \pm 0.2$ & $60.66 \pm 0.2$ & $\begin{array}{c}121.94 \pm 0.2 \\
(2: 01.94 \text { minutes) }\end{array}$ \\
\hline Trial 3 & $29.86 \pm 0.2$ & $78.84 \pm 0.2$ & $\begin{array}{c}136.14 \pm 0.2 \\
(2: 16.14 \text { minutes })\end{array}$ \\
\hline Trial 4 & $19.38 \pm 0.2$ & $64.71 \pm 0.2$ & $\begin{array}{c}133.01 \pm 0.2 \\
(2: 13.01 \text { minutes })\end{array}$ \\
\hline
\end{tabular}

The average and standard deviation of each point was also calculated and can be found in Table VI below. 
Table VI. Average Development Times for Rotational Device

\begin{tabular}{|c|c|c|c|c|c|}
\hline $\begin{array}{c}\text { Average } \\
\text { Time to } \\
\text { Detection } \\
\text { Pad (s) }\end{array}$ & $\begin{array}{c}\text { Standard } \\
\text { Deviation } \\
\text { of Time to } \\
\text { Detection } \\
\text { Pad (s) }\end{array}$ & $\begin{array}{c}\text { Average } \\
\text { Time to } \\
\text { Control } \\
\text { Pad (s) }\end{array}$ & $\begin{array}{c}\text { Standard } \\
\text { Deviation } \\
\text { of Time to } \\
\text { Control } \\
\text { Pad (s) }\end{array}$ & $\begin{array}{c}\text { Average } \\
\text { Time to } \\
\text { Absorbent } \\
\text { Pad (s) }\end{array}$ & $\begin{array}{c}\text { Standard } \\
\text { Deviation of } \\
\text { Time to } \\
\text { Absorbent } \\
\text { Pad (s) }\end{array}$ \\
\hline $30.36 \pm 0.2$ & 9.72 & $72.17 \pm 0.2$ & 11.31 & $128.66 \pm 0.2$ & 6.98 \\
\hline
\end{tabular}

\subsection{Enzyme Assay Development}

\subsubsection{Antibody Reactivity Analysis}

The first assay performed on the device was to determine the reactivity between the antigen and the antibodies. A photograph of the device was taken after TMB had been added, as seen in Figure 40.

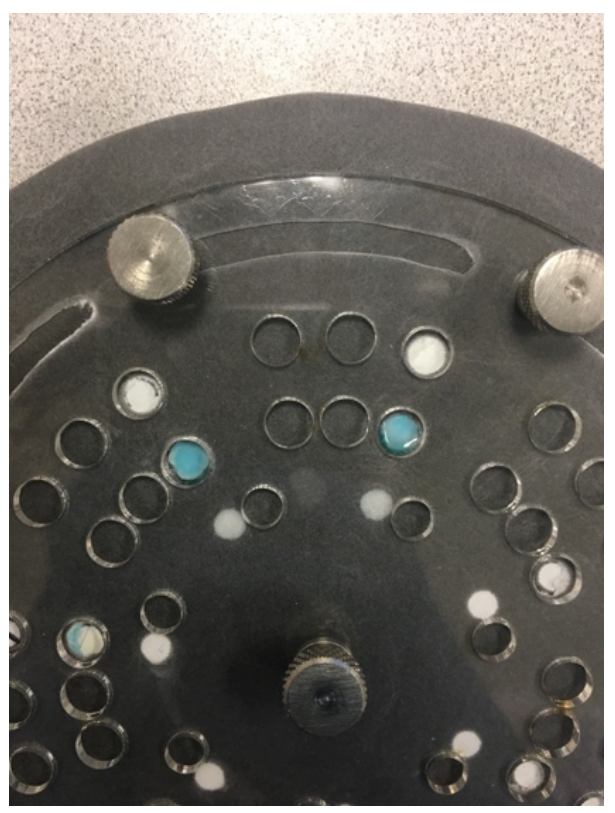

Figure 40. Colorimetric result of antibody analysis test

The image in the above figure was opened in ImageJ and the intensity of each sample and control was taken in order to determine a signal-to-noise ratio, calculated by dividing the sample intensity by the control intensity. The two tests were run utilizing the same 
protocols, so the percent difference between the two ratios was taken in order to quantify the repeatability of the results. The average and standard deviation are below in Table VII.

Table VII. Color Intensity of Initial Reactivity Tests

\begin{tabular}{|c|c|c|}
\hline & Intensity & Signal-to-Noise Ratio \\
\hline Trial 1 & 50.385 & 3.5613 \\
\hline Trial 2 & 48.054 & 3.3965 \\
\hline $\begin{array}{c}\text { Control } \\
\text { (background) }\end{array}$ & 14.148 & 1.0000 \\
\hline \hline \multicolumn{2}{|r|}{ Percent Difference } & 4.7359 \\
\hline \multicolumn{2}{|r|}{ Average } & 3.4789 \\
\hline & Standard Deviation & 0.1165 \\
\hline
\end{tabular}

\subsubsection{Streptavidin Characterization}

A device was run with two types of streptavidin (mutant and native) in triplicate placed on the detection pad prior to loading with a biotinylated anti-S100B antibody and tested against with $1 \mathrm{ng} / \mathrm{ml} \mathrm{S100B}$. The device can be seen in the figure below.

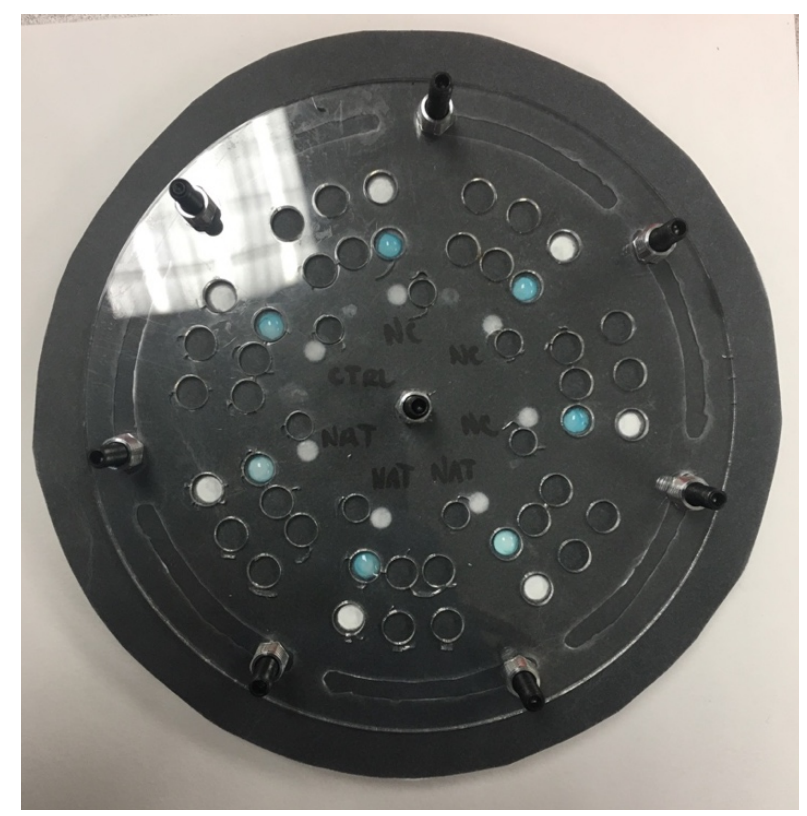

Figure 41. Device used to characterize mutant vs native streptavidin 
ImageJ was used to analyze the intensity of each detection pad and the average for each trial was taken, along with the standard deviation, as displayed in Table VIII below.

Table VIII. ImageJ Analysis of Streptavidin Assay

\begin{tabular}{|c|c|c|c|c|}
\hline & $\begin{array}{c}\text { Average } \\
\text { Intensity }\end{array}$ & $\begin{array}{c}\text { Average } \\
\text { Signal-to-Noise }\end{array}$ & $\begin{array}{c}\text { Standard } \\
\text { Deviation }\end{array}$ & $\mathbf{9 5 \%}$ CI \\
\hline $\begin{array}{c}\text { Mutant } \\
\text { Streptavidin }\end{array}$ & 85.51 & 2.41 & 0.139 & $(2.037,2.776)$ \\
\hline $\begin{array}{c}\text { Native } \\
\text { Streptavidin }\end{array}$ & 67.22 & 1.89 & 0.295 & $(1.522,2.261)$ \\
\hline Control & 74.69 & 2.10 & -- & $(1.462,2.742)$ \\
\hline Background & 35.54 & 1.00 & -- & \\
\hline
\end{tabular}

After conducting an ANOVA on the dataset, it was determined that the p-value was

0.121 , which, since it is larger than 0.05 , means that the data is not statistically different.

\subsubsection{Optimization of Detection Antibody}

This experiment was used to determine the appropriate concentration of detection antibody to load onto the conjugate pad. Figure 42 below shows the final result of one of the trials of the experiment.

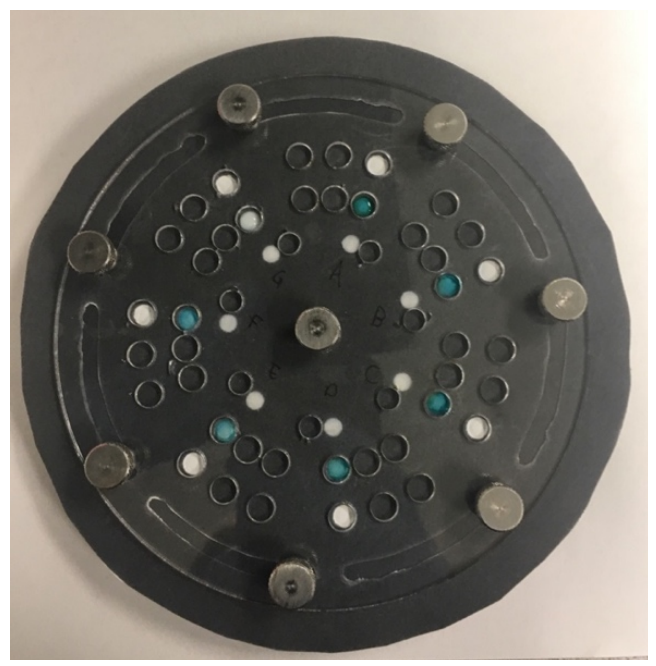

Figure 42. Colorimetric result for trial one of optimization of detection antibody tests 
The times were also taken for each device in order to compare to the fluid routing trials and can be seen in the table below.

Table IX. Time for Development of Detection Antibody Optimization Trials

\begin{tabular}{|c|c|c|c|}
\hline & $\begin{array}{c}\text { Time to Detection Pad } \\
\text { (seconds) }\end{array}$ & $\begin{array}{c}\text { Time to Control Pad } \\
\text { (seconds) }\end{array}$ & $\begin{array}{c}\text { Time to Absorbent Pad } \\
\text { (seconds) }\end{array}$ \\
\hline Trial 1 & $37.45 \pm 0.2$ & $216.32 \pm 0.2$ & $\begin{array}{c}366.87 \pm 0.2 \\
(6: 08.87 \text { minutes })\end{array}$ \\
\hline Trial 2 & $25.18 \pm 0.2$ & $154.51 \pm 0.2$ & $\begin{array}{c}282.27 \pm 0.2 \\
(4: 42.27 \text { minutes })\end{array}$ \\
\hline Trial 3 & $41.95 \pm 0.2$ & $175.25 \pm 0.2$ & $\begin{array}{c}300.67 \pm 0.2 \\
(5: 00.67 \text { minutes })\end{array}$ \\
\hline
\end{tabular}

The average and standard deviation of each point was also calculated and can be found in Table $\mathrm{X}$ below.

Table X. Average Development Times for Detection Antibody Optimization Trials

\begin{tabular}{|c|c|c|c|c|c|}
\hline $\begin{array}{c}\text { Average } \\
\text { Time to } \\
\text { Detection } \\
\text { Pad (s) }\end{array}$ & $\begin{array}{c}\text { Standard } \\
\text { Deviation } \\
\text { of Time to } \\
\text { Detection } \\
\text { Pad (s) }\end{array}$ & $\begin{array}{c}\text { Average } \\
\text { Time to } \\
\text { Control } \\
\text { Pad (s) }\end{array}$ & $\begin{array}{c}\text { Standard } \\
\text { Deviation } \\
\text { of Time to } \\
\text { Control } \\
\text { Pad (s) }\end{array}$ & $\begin{array}{c}\text { Average } \\
\text { Time to } \\
\text { Absorbent } \\
\text { Pad (s) }\end{array}$ & $\begin{array}{c}\text { Standard } \\
\text { Deviation of } \\
\text { Time to } \\
\text { Absorbent } \\
\text { Pad (s) }\end{array}$ \\
\hline $34.86 \pm 0.2$ & 8.67 & $182.02 \pm 0.2$ & 31.45 & $316.60 \pm 0.2$ & 44.49 \\
\hline
\end{tabular}

The image was also analyzed using ImageJ, as specified in the Methods. The results of the signal-to-noise ratios of the samples compared to the blank controls on each trial are plotted below. 


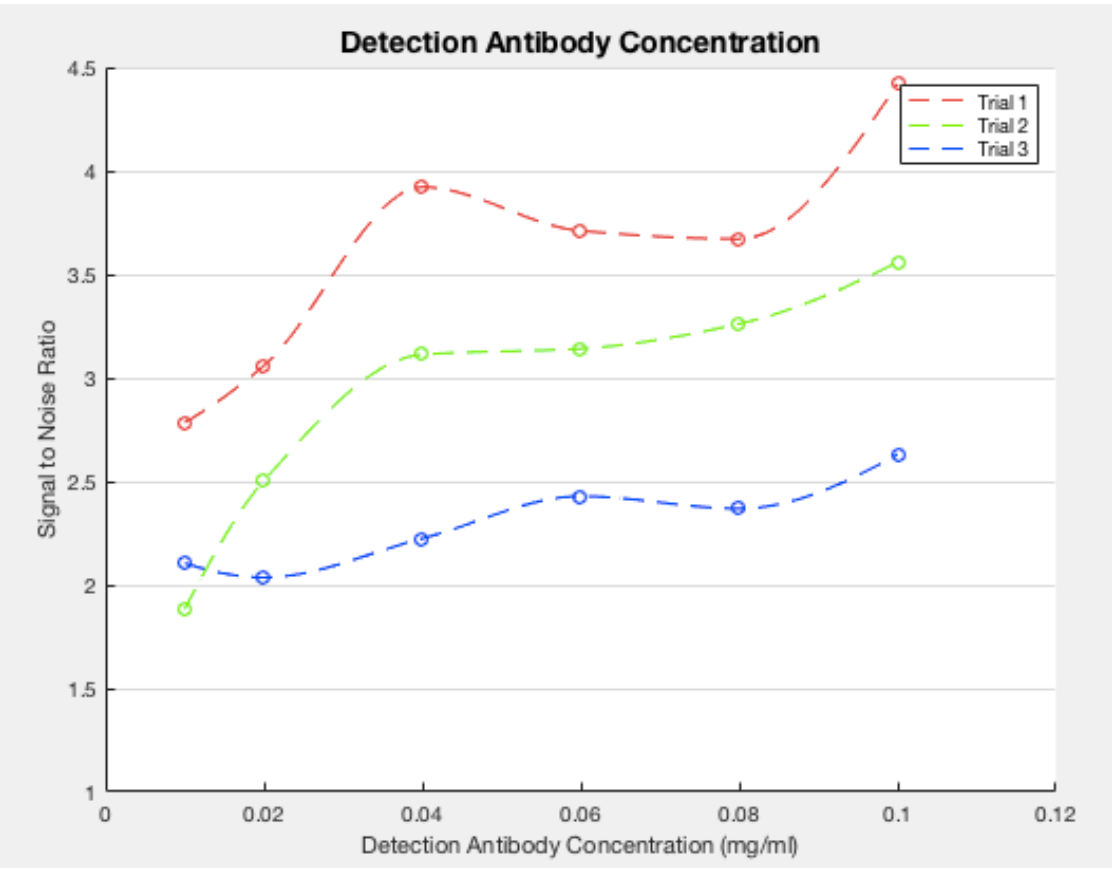

Figure 43. All trials of signal quality in dependence of detection antibody concentration

The signal to noise ratios for each trial were averaged for each concentration and the results are displayed in Figure 44 below, including error bars \pm 1 SD.

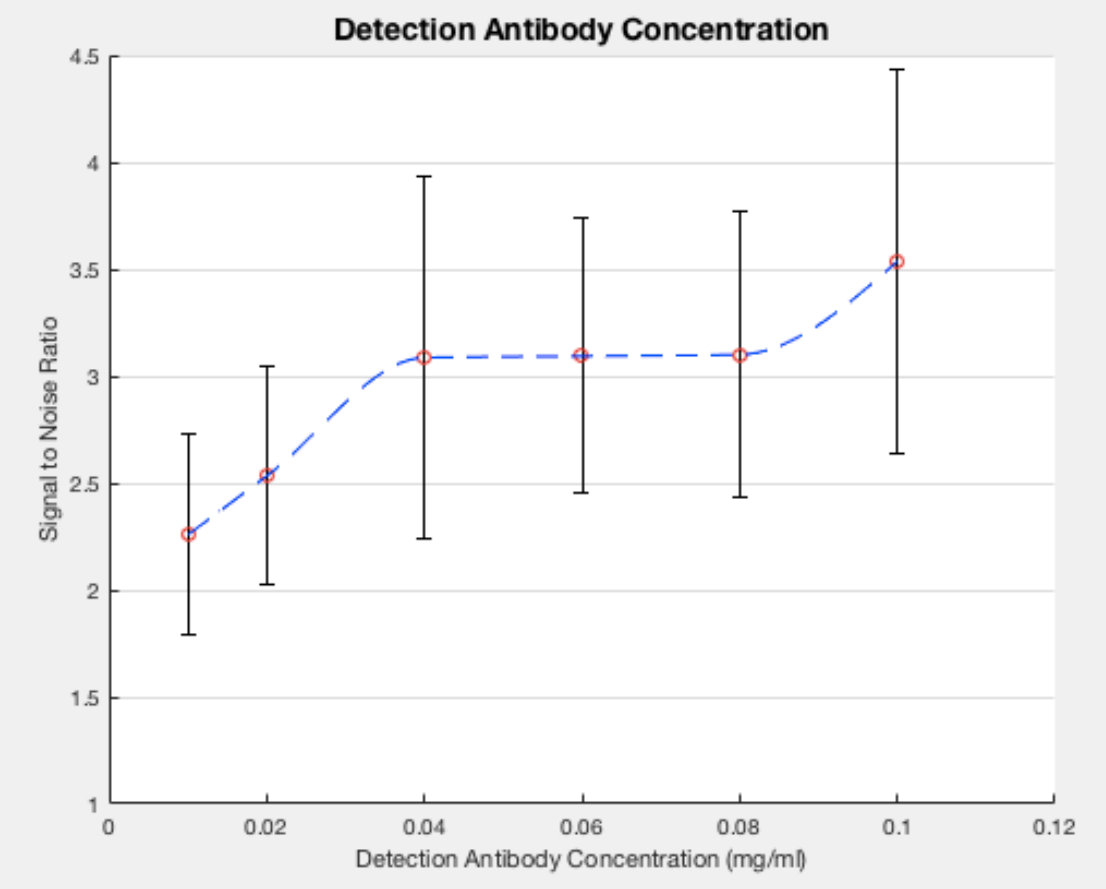

Figure 44. Signal quality in dependence of detection antibody concentration 


\subsubsection{Determination of Limit of Detection}

The results for the response of the device to different analyte concentrations is shown below.
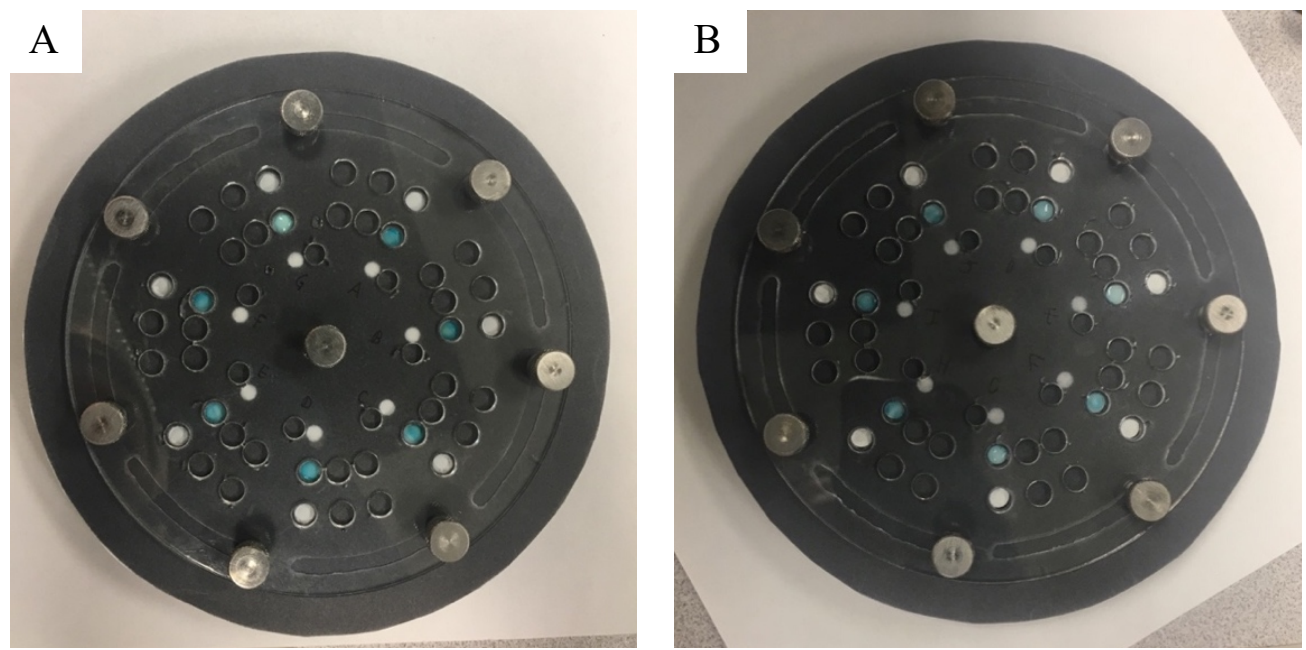

Figure 45. Assay response to different analyte concentrations, A) high set of concentrations and $B$ ) low set of concentrations

Some of these devices were also run using the control antibody on the control pad (furthest out ring of circles), which are used to determine if the test ran properly with enough sample. This can be seen in Figure 46 below, where all of the detection and control pads are blue, indicating a positive test that ran correctly.

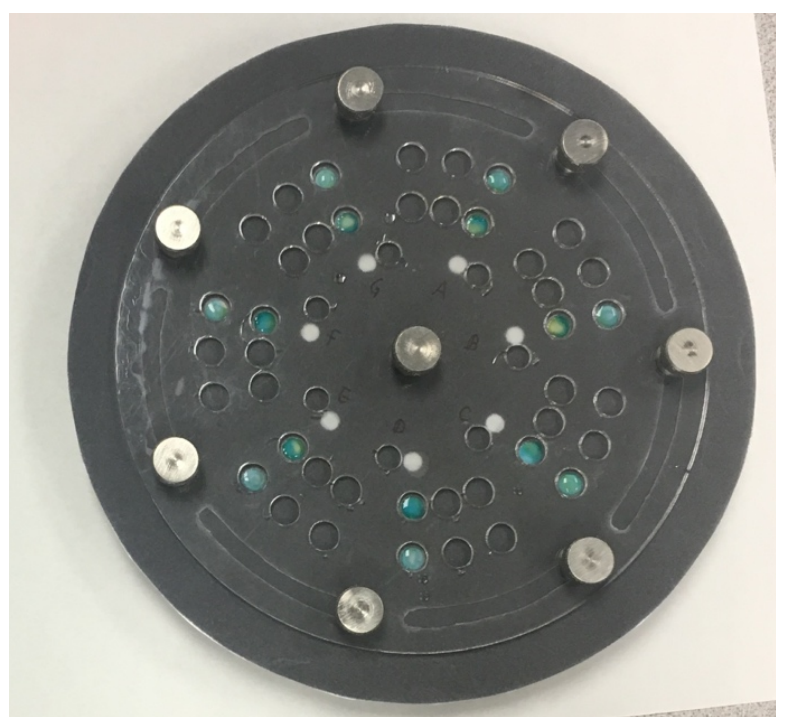

Figure 46. Device showing control pad functionality 
The times were also taken for each device in order to compare to the fluid routing trials and can be seen in the table below, \pm reaction time.

Table XI. Development Times for Dose Response Device Trials

\begin{tabular}{|c|c|c|c|}
\hline & $\begin{array}{c}\text { Time to Detection Pad } \\
\text { (seconds) }\end{array}$ & $\begin{array}{c}\text { Time to Control Pad } \\
\text { (seconds) }\end{array}$ & $\begin{array}{c}\text { Time to Absorbent Pad } \\
\text { (seconds) }\end{array}$ \\
\hline Trial 1 & $32.86 \pm 0.2$ & $188.40 \pm 0.2$ & $\begin{array}{c}452.67 \pm 0.2 \\
(7: 32.67 \text { minutes })\end{array}$ \\
\hline Trial 2 & $30.87 \pm 0.2$ & $215.12 \pm 0.2$ & $\begin{array}{c}328.38 \pm 0.2 \\
(5: 28.38 \text { minutes })\end{array}$ \\
\hline Trial 3 & $27.63 \pm 0.2$ & $312.26 \pm 0.2$ & $\begin{array}{c}504.15 \pm 0.2 \\
(8: 24.15 \text { minutes })\end{array}$ \\
\hline
\end{tabular}

The average and standard deviation of each point was also calculated and can be found in Table XII below.

Table XII. Average Development Times for Dose Response Trials

\begin{tabular}{|c|c|c|c|c|c|}
\hline $\begin{array}{c}\text { Average } \\
\text { Time to } \\
\text { Detection } \\
\text { Pad (s) }\end{array}$ & $\begin{array}{c}\text { Standard } \\
\text { Deviation } \\
\text { of Time to } \\
\text { Detection } \\
\text { Pad (s) }\end{array}$ & $\begin{array}{c}\text { Average } \\
\text { Time to } \\
\text { Control Pad } \\
\text { (s) }\end{array}$ & $\begin{array}{c}\text { Standard } \\
\text { Deviation } \\
\text { of Time to } \\
\text { Control } \\
\text { Pad (s) }\end{array}$ & $\begin{array}{c}\text { Average } \\
\text { Time to } \\
\text { Absorbent } \\
\text { Pad (s) }\end{array}$ & $\begin{array}{c}\text { Standard } \\
\text { Deviation of } \\
\text { Time to } \\
\text { Absorbent } \\
\text { Pad (s) }\end{array}$ \\
\hline $30.45 \pm 0.2$ & 2.64 & $238.59 \pm 0.2$ & 65.18 & $428.40 \pm 0.2$ & 90.36 \\
\hline
\end{tabular}

The images were also analyzed using ImageJ. The signal to noise ratios for each trial were averaged for each concentration and the results are displayed below. The data was fitted to a Four parameter logistic (4PL) curve which is commonly used to display ELISA results because it follows a sigmoidal "S" shape which appropriately approximates the colorimetric change across concentration ranges. MATLAB was used to fit the curve to the expression [61]:

$$
y=d+\frac{a-d}{\left[1+\left(\frac{x}{c}\right)^{b}\right]}
$$


Wherein:

a is the minimum value that can be obtained; expected at 0 dose concentration;

$\mathrm{d}$ is the maximum value that can be obtained;

$\mathrm{c}$ is the point of inflection; and

b is Hill's slope of the curve at the inflection point [62].

The MATLAB code returned the following results which can be seen plotted with the data compiled from the dose response trials, \pm standard deviation, corrected for outliers. The raw data can be found in the Appendix.

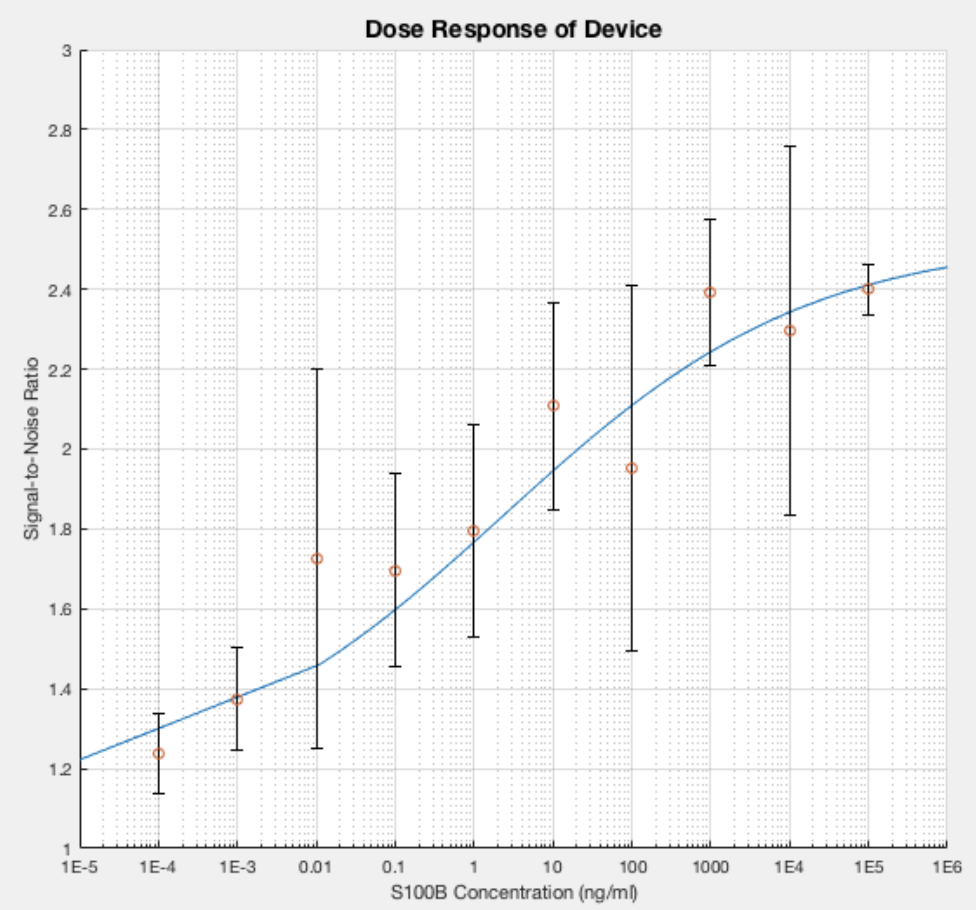

Figure 47. Dose response for different analyte concentrations fit to a $4 \mathrm{PL}$ regression curve

The equation for the 4PL fit was determined, shown below, with an $\mathrm{R}^{2}$ value of 0.9365 indicating a good fit to the data.

$$
y=2.527+\frac{1.141-2.527}{1+\left(\frac{x}{2.367}\right)^{0.2246}}
$$


In order to determine the LOD, the average intensity value of the blanks used was added to three times the standard deviation of the blank [63]:

$$
L O D=A_{0}+3 * \sigma
$$

This intensity LOD value was then plugged into the returned 4PL equation for the intensity versus concentration of S100B graph, found in the Appendix, which returned a value for the LOD in $\mathrm{ng} / \mathrm{ml}$. It was determined that the LOD for the device is $147.9 \mathrm{pg} / \mathrm{ml}$. Since human S100B has a molecular weight of $12.5 \mathrm{kDa}$, the LOD was calculated to be 11.83 pM $\left(1.1832 \times 10^{-11} \mathrm{M}\right)[63]$.

\subsubsection{Analysis of Troponin-Based Assay}

The results for the paper-based microfluidic device enabled to detect troponin can be seen in Figure 48 below.

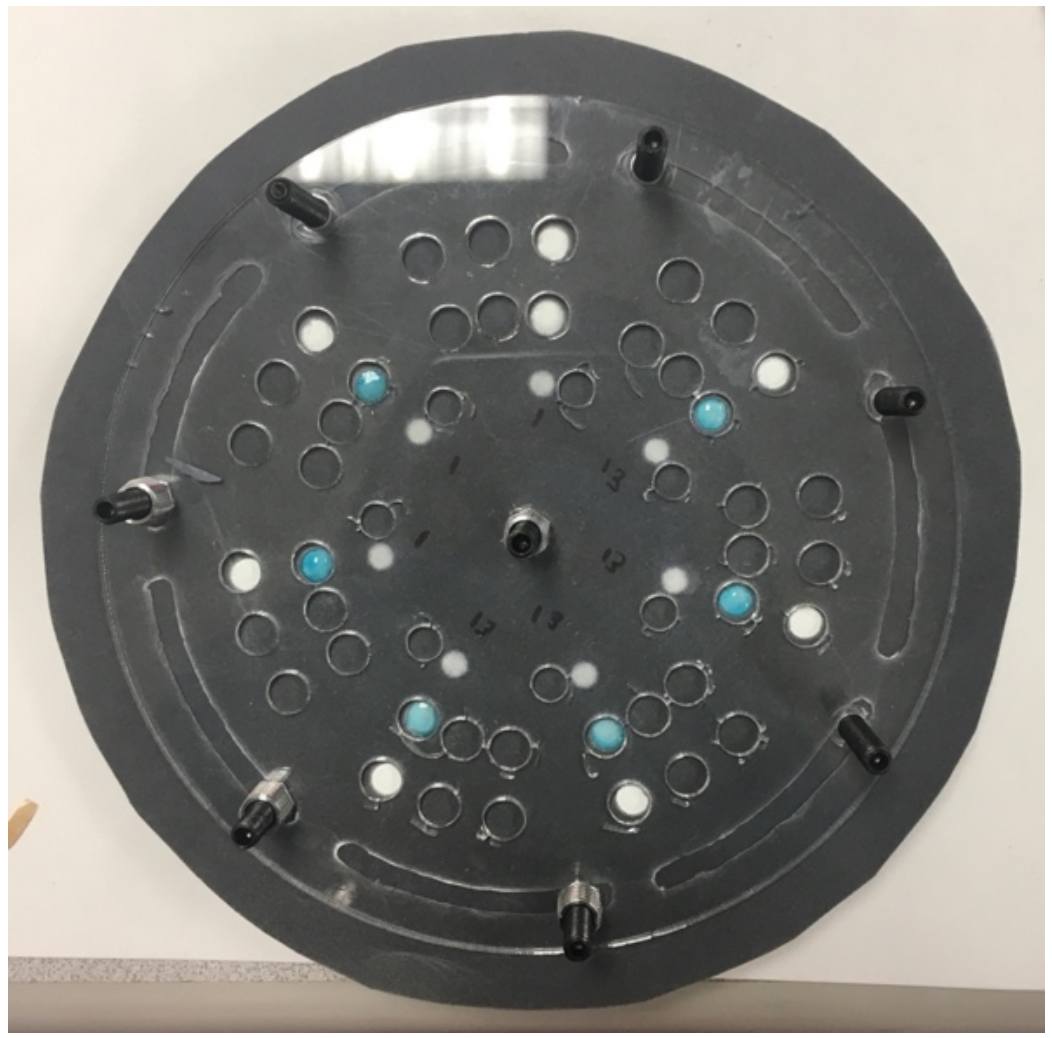

Figure 48. Results of troponin-enabled detection assay device 
Six of the trials were run with protein while the seventh trial was run with PBS as a background control. The results from the ImageJ analysis can be seen in the table below.

Table XIII. ImageJ Analysis Results for the Troponin-Enabled Assay

\begin{tabular}{|c|c|c|c|}
\hline & $\begin{array}{c}\text { Average } \\
\text { Intensity }\end{array}$ & $\begin{array}{c}\text { Average } \\
\text { Signal-to-Noise }\end{array}$ & $\begin{array}{c}\text { Standard } \\
\text { Deviation }\end{array}$ \\
\hline $\mathbf{1 . 6} \mathbf{~ m g / m l ~ C a p t u r e ~ A b}$ & 78.10 & 1.49 & 0.2932 \\
\hline $\mathbf{0 . 1} \mathbf{~ m g} / \mathbf{m l}$ Capture Ab & 72.90 & 1.39 & 0.0969 \\
\hline Control (background) & 52.41 & 1.00 & -- \\
\hline
\end{tabular}

An ANOVA was also conducted on this data set and returned a p-value of 0.338 , which means that, at a significance level of 0.05 , the data was not statistically significantly different. 


\section{DISCUSSION}

This section will be used to analyze the data presented in the previous section in order to draw conclusions about the fabrication and functionality of the assay and its housing.

\subsection{Analysis of Previous Work}

The previous design (Holler, 2016) printed on cellulose was functionally able to route red dye through each layer, which could be seen in the Results. This verifies the ability to route fluid through the device successfully. This result provides a foundation for the assay design which can be built upon in order to improve the functionality of the ELISA-like assay; however, Holler was unable to integrate the ELISA-like assay onto the device, likely due to her protocol for reagent storage on and resolubilization from the conjugate pads. The aim of the rest of the discussion will be to determine if the ELISAlike assay could be successfully implemented on the updated device design.

\subsection{Dye Tests}

Several trials of each dye test were performed in order to gather accurate information about the functionality of the fluid flow pathway of the assay. While 3D $\mu$ PADs have been created using wax-printing techniques and the base format of routing up and down through layers had been demonstrated by Holler (2016), the design change was substantial enough to warrant testing before moving forward with reagent incorporation. 


\subsubsection{Single Sample Device - Taped Assembly}

The first trials of fluid flow were completed using the design wax-printed on cellulose and taped together onto a plastic petri dish to aid in layer-to-layer connectivity. The initial trials were not successful, as the fluid flow was stopped after the detection pad, but before the control pad, in the area marked in Figure 49A below. This can also be seen in Figure 49B, where the red dye stopped in the fluid routing layer and did not reach the fourth layer or the absorbent pad.
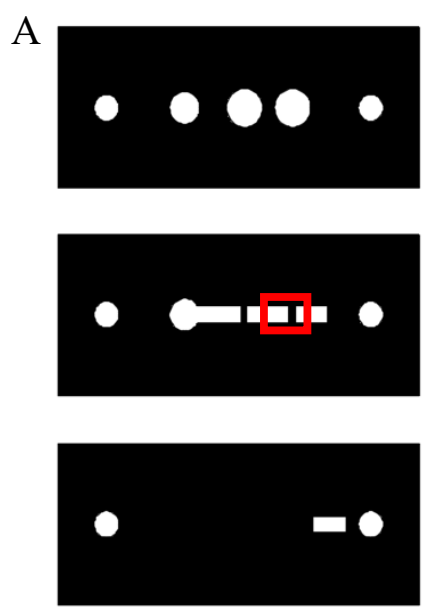
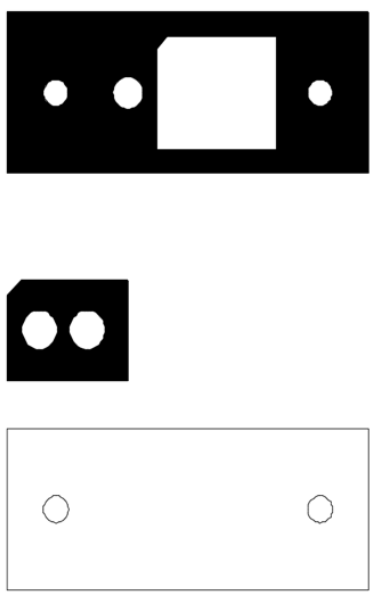

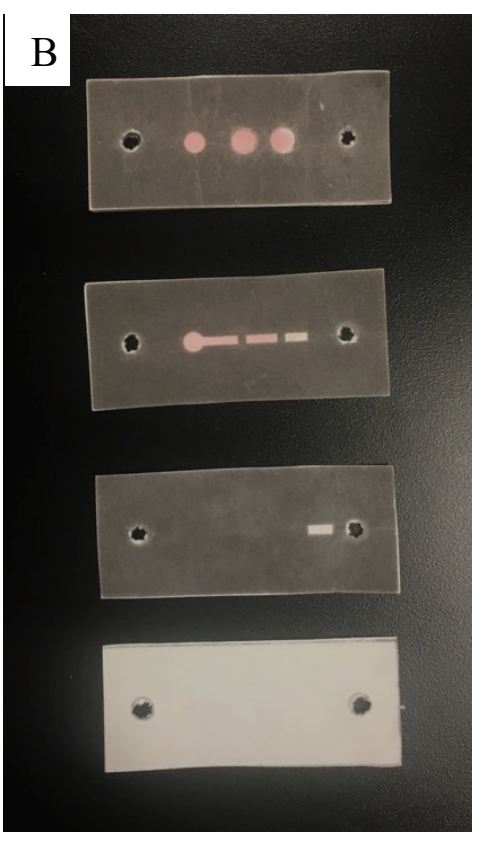

Figure 49. Single sample fluid flow design with A) flow barrier area marked in red on AutoCAD design and $B$ ) results from an incomplete trial

Across all of the trials, there was inconsistency with lining up the channels between layers and providing ample pressure to ensure that the layers were in contact with each other in the proper places. If the layers were not placed together precisely, the fluid would become trapped at a barrier, where it would be unable to transition up or down to continue to flow laterally. Due to the region of impedance, it was determined that the wax bridge was too thick and was causing a blockage in the flow pathway if the layers became misaligned, 
which would have been further perpetuated without sufficient pressure externally to provide layer connectivity. Due to this, the thicknesses of the wax bridges were decreased, and more care was taken to precisely align the layers with tape.

As can be seen in the Results section, the design of the single sample device was eventually able to successfully route the red dye through from the sample pad, up to the detection pad and subsequently the control pad, flowing down to the absorbent pad. Since the red dye can be seen in each exposed pad, as well as within the hydrophilic cellulose channels when the device was taken apart, it was deemed a successful trial of the design. There were no issues seen with fluid "overflowing" from the paper channels over onto the wax areas between layers. A total development time of 2:18.54 minutes was initial validation that the device would be able to quickly bring the patient sample through to the correct reagents in order to yield a positive or negative result.

\subsubsection{Single Sample Device - Housing Assembly}

With the challenges faced when prototyping the single channel design with tape between layers, it was determined that a housing would need to be created to properly demonstrate the functionality of the intended final design. The housing would help both with providing ample pressure throughout the device, as well as aiding in the alignment of each layer. The screws would be used to hold the top and bottom housing components to each other, and when the screw holes in each layer were cut out and inserted over the screws, it became increasingly easier to assemble to device correctly, since the screws prevented shifting of layers and were used as guides for how the layers were intended to be placed. 
Once the red dye fluid flow trails were conducted in this housing, more meaningful data was able to be obtained concerning development time and overall success in fluid routing. As can be seen in the Results, the dye front flowed successfully through to device across all three trials. The mean development time was 2:40.91 minutes with a standard deviation of \pm 42.72 seconds. The first trial resulted in the longest development time, whereas the subsequent two trials had very similar development times, at approximately 2 minutes. The largest standard deviation occurred in the time between the control pad to the absorbent pad, where the other two time points had relatively small standard deviations for the three trials. This could be because the first trial did not have proper alignment between the routing layers (layers three and four) which rely on the overlap of two thin rectangular regions. Therefore, a shift in the alignment would most noticeably impact the flow down to the absorbent pad due to the smaller area of overlap when compared to elsewhere in the device. However, since the speed of the device was sufficiently fast in comparison to existing diagnostic techniques, and it was not intended to be optimized further, the single sample design was considered finalized and the dimensions were transformed into a sevensample circular device in a rotating assembly.

\subsubsection{Rotating Assembly}

Upon finalizing the design for the single sample design, the rotating design was printed and assembled and tested for fluid flow as well, to ensure that the translation between one sample to seven samples maintained the functionality of the device. These trials were run using a mocked-up housing made from plastic sheeting that was faster and cheaper to make than the final acrylic housing but would still give accurate data. All four 
trials of the rotating design printed on cellulose paper were successful, with fluid routing efficiently between each layer down to the absorbent pad. The average development time was determined to be 2:08.66 minutes, with a standard deviation of \pm 6.98 . This time data deviated less between each trial than the single sample flow design, which would indicate that the extra points of pressure from the seven screws along the outside of the device and the single screw in the center created better pressure and alignment between layers. When taken apart, the red dye could be seen in all intended flow paths, including the first sample channel and the three subsequent wash step channels, also run with red dye. These trials were promising for the success of the device when considering the ability of the sample to reach each area for the specific reaction step and flow entirely through the device to fully develop a signal that could be read by the user when the proper reagents were incorporated into the device.

\subsection{Enzyme Assay Development}

After successfully designing the device to route fluid through the intended pathways at a rapid pace, the next step was to incorporate the ELISA-like reagents onto the platform so that a diagnostic test could be run from a sample. These experiments used the full set up of the device, including a nitrocellulose detection pad as opposed to the cellulose which had been used in the fluid flow experiments, a separate glass fiber conjugate pad for the HRP-conjugated antibody, and the inclusion of the reagents for reactivity. 


\subsubsection{Antibody Analysis}

In the first trial of the ELISA-like assay incorporation of the device, two identical tests were run to determine if the antibodies and antigen purchased were reactive and if the test would be able to return a discernable color change with the addition of the HRP substrate, TMB. This test was also used to determine the precision of the ImageJ analysis method. As seen in the Results, the incorporation of on-board reagents was successful, and the test was able to run to completion, as indicated by the blue color on the detection pads. The sample - PBS spiked with $0.1 \mathrm{mg} / \mathrm{ml} \mathrm{S100B} \mathrm{-} \mathrm{was} \mathrm{able} \mathrm{to} \mathrm{resolubilize} \mathrm{the} \mathrm{detection}$ antibody on the conjugate pad, something which was not realized in the previous work (Holler, 2016), and the fluid front could carry the detection antibody and S100B complex to the detection pad to bind to the capture antibody and return a visual result when reacted with TMB. When comparing the values returned for intensity from the ImageJ intensity analysis, the percent difference between the two was low (below 5\%) and indicated, along with the standard deviation $(0.1165)$ that the software was able to return results in an accurate and repeatable fashion.

These two trials were considered successes because now the protocol used to set up the device was determined to be appropriate in yielding the desired result, and the parameters could be changed in subsequent experiments in order to optimize the device.

\subsubsection{Streptavidin Characterization}

The necessity of the streptavidin + biotin binding reaction for the functionality of the device was analyzed in an experiment which compared final color intensity and signalto-noise of the assay run with $\mathrm{S} 100 \mathrm{~B}$ against a biotinylated antibody bound to mutant 
streptavidin, native streptavidin, and a control without streptavidin. After an ANOVA was conducted on the data, the p-value was determined to be 0.121 , which is larger than 0.05 , meaning that the average values for signal-to-noise were not statistically different from one another. Therefore, with the three trials, it can be concluded that the type of streptavidin, or lack thereof, did not have a significant effect on the signal. This is interesting because, in theory, the nitrocellulose only non-specifically binds to the antibodies, leaving them susceptible to being washed off of the detection pad. Therefore, the streptavidin + biotin pair would be used to encourage binding to the nitrocellulose and minimize sample loss. Further testing would need to be done with a larger data set in order to determine if the mutant vs native streptavidin has a functional difference with binding between nitrocellulose and the biotinylated antibody.

\subsubsection{Optimization of Detection Antibody}

The concentrations and quantities of reagents used in the first experiment were drawn from research into literature which used a similar principle of incorporation of an ELISA-like assay onto a paper-based device. These values were used to determine a baseline for the feasibility of obtaining a visible signal, and once that was achieved, changes were made to the protocol that would allow the device to run off of minimal amounts of reagents in order to reduce costs and eliminate background noise caused specifically by excessive antibodies.

In this experiment, the concentration of the HRP-conjugated detection antibody was varied in order to determine the critical concentration where there is sufficient antibody to produce an accurate signal by binding with a majority of the antigen but there is not too 
much so that there is surplus of unbound antibody on the detection pad which is still returning a positive result by catalyzing the TMB. When the detection antibody is at too low of a concentration, the antigen will not be bound and only a portion of it would return a signal, meaning that the device would return a signal that indicates that the antigen concentration in the sample is lower than it is in reality. When the detection HRPconjugated antibody is at too high of a concentration, any unbound antibody has a higher chance of nonspecifically binding onto the detection pad after the wash steps and producing a color change, indicating a positive result when, in reality, no antigen is bound, leading to a result which indicates that the antigen concentration in the sample is higher than it is in reality. There is, therefore, a concentration of HRP-conjugated detection antibody which will allow for the accurate and specific binding of the antigen and only result in a colorimetric result when the full immunocomplex is created on the detection pad.

The devices which were run with the control pad suffered from timing issues with the color development due to the fact that the user needed to manually pipette 14 regions with TMB as opposed to only 7 in the tests without a control pad. Since the control pad would not be analyzed for color intensity and is functioning as a "yes/no" result due to blue color/no color change, the detection pads were pipetted first and a photo was taken before the TMB was pipetted onto the control pad to test for device functionality.

The time for development of each trial was taken in order to quantify the time for detection. The average time for the sample to completely flow to the absorbent pad was calculated to be 5:16.60 minutes with a standard deviation of \pm 44.49 seconds between each trial. This speed was slower than the development times seen in the previous fluid flow trials likely due to the inclusion of the glass fiber conjugate pad and the nitrocellulose 
layer as compared to the fluid flow tests which were run entirely on cellulose paper. The detection antibody on the conjugate pad was also dried down in a PBS solution with $10 \%$ sucrose to aid in protein stability, and the sucrose would have impeded the flow of the sample through the device because the sample must resolubilize the sucrose before continuing on to the detection region [35]. While this slowed down the development times, it would theoretically be a positive because the sample and the analyte within would have a longer incubation time with the detection antibody on the conjugate pad than it would without the sucrose.

All of the trials for this experiment were graphed and displayed in the Results. As seen in this graph, each trial follows the same trend but at different magnitudes of signalto-noise. It can be concluded that the intensity of the sample area, as determined by ImageJ, is highly dependent on the photograph taken, specifically the lighting. However, since the plots follow the same trend, it can also be inferred that the data was consistent between trials, even though the actual numbers were not the same.

As can be seen in the Results, the graph of detection antibody versus average signalto-noise ratio increased when moving from a low concentration of $0.01 \mathrm{mg} / \mathrm{ml}$ up to 0.04 $\mathrm{mg} / \mathrm{ml}$. This is in accordance with the expectations - the more HRP-conjugated antibody on the conjugate pad, the higher the intensity of the signal and therefore the higher the signal relative to the noise. Then, between $0.04 \mathrm{mg} / \mathrm{ml}$ and $0.08 \mathrm{mg} / \mathrm{ml}$, there is a plateau in the signal-to-noise ratio, which indicates that the critical concentration had been reached and the signal-to-noise ratio was not improving with the addition of more detection antibodies. Then, at a concentration of $0.1 \mathrm{mg} / \mathrm{ml}$, the signal-to-noise ratio increased dramatically, which is indicative of unbound HRP-conjugated detection antibody 
nonspecifically binding to the nitrocellulose and not getting fully washed away by PBS and therefore still resulting in what is read as a positive signal, when in actuality, no antigen has been bound. When looking at the raw data, it was determined that a high signal-tonoise ratio for one trial of $0.08 \mathrm{mg} / \mathrm{ml}$ was elevating the average value up to the $0.04 \mathrm{mg} / \mathrm{ml}$ and $0.06 \mathrm{mg} / \mathrm{ml}$ concentrations, when it was actually likely a bit lower, indicating a decrease in the signal relative to the background noise before the increase up to $0.1 \mathrm{mg} / \mathrm{ml}$. Therefore, it was determined that the optimal concentration of the HRP-conjugated detection antibody was $0.05 \mathrm{mg} / \mathrm{ml}$, half as much as the highest signal response and still within the plateau region of the graph as the average between 0.04 and $0.06 \mathrm{mg} / \mathrm{ml}$.

The graph also shows error bars \pm 1 standard deviation. These bars are considerably large due to the fact that the intensity values are calculated from a picture taken of each device at the end of the trial under variable lighting, as briefly discussed above. This means that while there is consistency in intensity within each trial because the photo is analyzed in reference to a control which is on the device, there is inherent inconsistency between trials for the intensity values. This is why the trend seen in the signal-to-noise data for each trial was considered as opposed to purely the values for intensity. In essence, the average, the $+1 \mathrm{SD}$, and $-1 \mathrm{SD}$ values respectively correspond to each trial conducted and the variability was expected but does not affect the results.

\subsubsection{Determination of Limit of Detection}

The final round of experiments conducted were to determine the limit of detection (LOD) of the device. This is important because the LOD is the lowest concentration of protein which can be accurately and repeatedly differentiated from a blank control. The 
LOD is a measure of the functionality of the clinical ability of the device. The LOD must be lower than the concentration of protein expected to be expressed in the patient sample when the disease or pathology is present so that said disease can be detected and diagnosed.

These experiments varied the concentration of antigen (S100B) applied onto the sample pad in order to obtain a standard curve of antigen concentration versus signal-tonoise or intensity, theoretically resulting in a sigmoidal shape. The reason for this shape is that the concentrations of antigen become too low to detect at a certain concentration and too high to differentiate at another certain concentration, causing the concentrations at the extreme low and high concentrations to return the same intensity values and therefore the same signal-to-noise values. Effectively, there exists a linear range of antigen concentrations in which the assay can sensitivity and specifically differentiate the concentration.

In these experiments, the concentration of capture antibody was kept at $0.1 \mathrm{mg} / \mathrm{ml}$, the concentration of detection antibody was maintained at $0.05 \mathrm{mg} / \mathrm{ml}$, per the previous optimization experiments, and the concentration of the S100B was varied from 0.0001 $\mathrm{ng} / \mathrm{ml}$ to $100,000 \mathrm{ng} / \mathrm{ml}$ via ten 10 -fold dilutions. This was intended to be sufficient to cover the entire range of the detection spectrum so that the linear area could be found between the extreme high and low concentrations. It can be seen in Figure 45 that the tests ran successfully and resulted in the blue color. The same reaction occurred as described in the previous section: $\mathrm{PBS}+\mathrm{S} 100 \mathrm{~B}$ resolubilized the conjugate antibody and bound, then traveled to the detection pad to bind to the capture antibody for eventual catalysis of TMB by HRP. 
The times for three of the trials were also taken, resulting in an average time to the absorbent pad of 7:08.40 minutes with a standard deviation of \pm 90.36 seconds. Again, these times had large standard deviations between trials, likely due to misalignments between the layers. The fastest time to the absorbent pad recorded was 5:28.38 minutes, which, when including the rotation, wash steps, and TMB addition, amounts to approximately 15 minutes to run the entire device and return a colorimetric response. This is magnitudes faster than the current gold standard; CT scans can take up to 60 minutes and contrast solution must be injected to see an ischemic stroke [64].

Each trial was photographed immediately after introduction of the TMB and analyzed by ImageJ for RGB signal intensity. These results can be seen in Figure 47, where the results were plotted against the calculated signal-to-noise ratio determined by a control on each device run. A sigmoidal 4PL curve was fit to the data using MATLAB code [61]. There exists a clear trend following the expected sigmoidal shape which spans the entire concentration range, with a linear region in the center from $0.01 \mathrm{ng} / \mathrm{ml}$ to $1000 \mathrm{ng} / \mathrm{ml}$, which would correlate to an approximate detection range. It is important to consider that the device runs similar to an ELISA and a standard curve of known concentrations would need to be run with the sample so that the standard curve could be created, and the concentration of the sample extrapolated from that based on the relative intensity.

Further, the 4PL regression curve was fit to signal intensity vs S100B concentration to determine the LOD. As stated in the results, the LOD was determined to be $147.9 \mathrm{pg} / \mathrm{ml}$ $(11.83 \mathrm{pM})$. This is within the range of sensitivity for commercial ELISAs for human S100B, which are cited between $19.5 \mathrm{pg} / \mathrm{ml}(1.56 \mathrm{pM})$ to $0.31 \mathrm{ng} / \mathrm{ml}(24.8 \mathrm{pM})$ [65] [66]. Clinically, S100B has been cited to be a biomarker for ischemic stroke at levels above 
$1,030 \mathrm{pg} / \mathrm{ml}, 350 \mathrm{pg} / \mathrm{ml}$, and $115.03 \mathrm{pg} / \mathrm{ml}$ [67] [68] [69]. Therefore, the ELISA-like device developed for this thesis hold clinical significance in detecting S100B in order to diagnose ischemic stroke.

\subsubsection{Analysis of Troponin-Based Assay}

The final test which was conducted was an analysis of the device using a different set of proteins and antibodies used for a previous thesis (Holler, 2016). While the test was only to see if the proteins used in the past work were functional for use on a paper-based device and to determine if the designed device would be able to detect other proteins if the on-board reagents are changed, the signal-to-noise ratios were compared between the two concentrations of capture antibody. The p-value returned for the ANOVA was greater than the 0.05 significance level and therefore was not statistically significant. Therefore, the concentration of capture antibody on the detection pad was not significant on the signalto-noise result.

This test, however, did show that the device was able to return a readable result for the detection of troponin using antigens and antibodies from the previous thesis (Holler, 2016). These antibodies had been stored in $-20^{\circ} \mathrm{C}$ conditions for the past three years, and while the detection pad exhibited a color change, this could have been due to a false positive of non-specific binding of the detection antibody to the nitrocellulose. Further experimentation would need to be conducted with freshly purchased antigen-antibody pairings for improved validation. Beyond demonstrating the adaptability of the device to be used with different analytes with the specific on-board antibodies, this experiment was able to conclude that the likely reason that the previous work was unable to realize a fully functional device with on-board detection antibody was that the cellulose conjugate pad 
used by Holler (2016) is not suitable for antibody storage and eventual resolubilization with sample introduction. A more effective alternative would be the glass fiber conjugate pad used in this device which was able to hold the HRP-conjugated detection antibody, resolubilize it to allow it to react with the analyte and carry it onward to the detection pad where the capture antibody is bound for eventual detection with TMB. 


\section{CONCLUSION}

This thesis demonstrates the ability for a 3D paper-based microfluidic device to incorporate an ELISA-like test on its platform in order to detect clinically significant concentrations of S100B in order to diagnose ischemic stroke. A prototype of a low-cost, highly sensitive, and easy-to-use portable device was achieved with the incorporation of on-board reagents onto the paper platform. This device was created using wax printing hydrophilic channels onto cellulose and nitrocellulose paper in order to guide a sample across the appropriate pads storing the reagents required for an ELISA-like test.

First, dye tests were performed in order to characterize the dye flow functionality of the device itself in a single-sample format. This series of experiments verified that the design functioned as intended and was able to consistently and quickly route dye across the conjugate pad, detection pad, control pad, and down to the absorbent pad. These tests were performed with housing to further prototype the intended final design. The housing aided in layer alignment and provided uniform pressure across the device to ensure sufficient layer connectivity.

Further, the incorporation of reagents onto the paper-based device developed a successful three-step reaction, consisting of sample analyte binding to an HRP-conjugated detection antibody and a biotinylated capture antibody, using S100B as a model antigen for this test. The novel rotation of the device's layers was used to open up new channels which were used as wash steps to further increase the sensitivity of the test, washing away unbound antibodies and antigens. The device was optimized for the ideal concentration of detection antibody and the limit of detection was determined. Through these optimization 
experiments, an LOD of $11.83 \mathrm{pM}$ was achieved, which is within the range of current ELISA tests on the commercial market.

This device, while also appropriate for use in hospitals and clinics, has potential to be used in developing nations which would not normally have access to this type of diagnostic technology. The test is portable, requires no external power, is cheap and easy to produce and use, and can be analyzed using cell phone camera images, meeting all of the ASSURED criteria put in place by the WHO, and making it a diagnostic device which has the ability to spread advanced diagnostics across the globe.

Overall, this thesis was able to demonstrate ELISA-like functionality on a novel paper-based design to achieve low limits of detection which by displaying a blue signal which can be converted into analyte concentration in order to accurately and quickly diagnose an ischemic stroke, resulting in faster treatment and better patient outcomes. 


\section{FUTURE DIRECTIONS}

This section will describe potential future work on this paper-based ELISA-like device in order to further optimize the test and achieve lower limits of detection.

\subsection{Signal Enhancement}

The reason why ELISAs are able to be so sensitive is due to the signal amplification which is created by the reaction between HRP and TMB, turning the detection region blue, which can be read by a variety of software. The downfall of the paper-based system is the fact that reagents are left behind on the paper when travelling through the device. Further research could look into the effect of different blocking agents, wash reagents, and materials on the final color intensity. It is possible that the blocking of the device channels, and not just the conjugate and detection pads, would lead to a decrease in non-specific binding and therefore an increase in the signal intensity for a specific analyte concentration. A wash reagent other than PBS might yield better results by picking up and carrying more antibodies through the device. Finally, the material could be changed from cellulose to newer engineered options, such as the Whatman ${ }^{\circledR}$ Fusion 5, which is a proprietary membrane fabricated for fast wicking rates with no blocking required, due to it being a hydrophobic material [70].

Additionally, the substrate of the enzymatic reaction should be investigated further to determine which substrate would have the best performance on the paper-based system. This thesis used a fast kinetic rate TMB from abcam ${ }^{\circledR}$ which made it difficult to obtain a picture of the accurate development of the blue color when accounting for the time taken to pipette the TMB onto seven sample pads. Either a different TMB solution could be 
purchased or another HRP substrate entirely could be analyzed. In the same vein, the manner by which the HRP substrate is added onto the device could be optimized. It took approximately one minute to pipette the substrate onto each detection pad, which causes the development time of each sample to be different at the time that the photograph is taken for analysis. Perhaps the HRP substrate could be incorporated onto the device itself and resolubilized by the sample or from one pipetting step so that the substrate would reach and be able to react with the HRP on the detection pads at the same time.

\subsection{Multiplex Assay}

This device was only optimized to run a single sample to test for one analyte. In the future, it would be beneficial to be able to run several samples to test for several analytes in a multiplex fashion which would allow for incorporation of a protein panel onto the device for more accurate diagnosis of stroke, or other pathologies as desired. The same design as the one presented in this thesis could be used, only the antibodies on the conjugate pad and detection pad would need to be changed and optimized for each specific analyte to achieve a multiplex device.

\subsection{Long-Term Reagent Storage}

In order for the incorporation of the on-board reagents to be useful, they must have a proven long shelf life. Tests could be run in the future to determine how stable the onboard reagents are with respect to time, humidity changes, and temperature changes that the device would undergo in transportation. Additionally, more development could be put into the housing unit utilized for this device. While the current assembly did provide a 
platform to quickly run an assay, it left the sides open in order to allow for rotation of the top layer. This creates a non-sterile environment that would be required for the device. The top layer hanging outside of the housing also makes it susceptible to bending and ripping, rendering the device largely unusable. The housing should be designed to fully enclose the test while also allowing the user to rotate the top layer, perhaps by using a rotating wheel on the top side of the housing to "pull" the layer of paper in a circle as the wheel is rotated. The possibilities for improvement on this device are varied and would only serve to create a better test for detection of stroke, or other conditions, and create better outcomes for at-risk patients around the world. 


\section{REFERENCES}

[1] Landsverk M.L., Wong LJ.C. (2013). Clinical Molecular Diagnostic Techniques: A Brief Review. In: Wong LJ. (eds) Next Generation Sequencing. Springer, New York, NY

[2] Kurkela, Satu, and David W.g. Brown. (2009). Molecular Diagnostic Techniques. Medicine, vol. 37, no. 10, pp. 535-540., doi:10.1016/j.mpmed.2009.07.012.

[3] Netto, G. J., Saad, R. D., \& Dysert, P. A. (2003). Diagnostic molecular pathology: current techniques and clinical applications, part I. Proceedings (Baylor University. Medical Center), 16(4), 379-83.

[4] Strimbu, K., \& Tavel, J. A. (2010). What are Biomarkers? Current Opinion in HIV and AIDS, 5(6), 463-466.

[5] Horlock, Claire. "Enzyme-Linked Immunosorbent Assay (ELISA)." British Society f or Immunology, www.immunology.org/public-information/bitesizedimmunology/experimental-techniques/enzyme-linked-immunosorbent-assay.

[6] BosterBio. Four Types of ELISA Assay. (2017). www.bosterbio.com/newsletterarchive/20170728-which-elisa.

[7] Thermo Fisher. "Overview of ELISA.” Thermo Fisher Scientific - US, www.thermofisher.com/us/en/home/life-science/protein-biology/protein-biologylearning-center/protein-biology-resource-library/pierce-proteinmethods/overview-elisa.html\#2.

[8] Zhang, Wenbao \& Wen, Hao \& Li, Jun \& Lin, Renyong \& McManus, Donald. (2012). Immunology and Immunodiagnosis of Cystic Echinococcosis: An Update. Clinical \& developmental immunology. 2012. 101895. 10.1155/2012/101895.

[9] Mazzu-Nascimento, Thiago, et al. (2017). Improved Assessment of Accuracy and Performance Indicators in Paper-Based ELISA. Analytical Methods, vol. 9, no. 18, pp. 2644-2653., doi:10.1039/c7ay00505a.

[10] Terato, Kuniaki, et al. (2014). Preventing Intense False Positive and Negative Reactions Attributed to the Principle of ELISA to Re-Investigate Antibody Studies in Autoimmune Diseases. Journal of Immunological Methods, vol. 407, pp. 15-25., doi:10.1016/j.jim.2014.03.013.

[11] Xiao, Y., \& Isaacs, S. N. (2012). Enzyme-linked immunosorbent assay (ELISA) and blocking with bovine serum albumin (BSA)--not all BSAs are alike. Journal of immunological methods, 384(1-2), 148-51.

[12] Heilman, J. M., De Wolff, J., Beards, G. M., \& Basden, B. J. (2014). Dengue fever: 
a Wikipedia clinical review. Open medicine : a peer-reviewed, independent, openaccess journal, 8(4), e105-e115.

[13] Koczula, K. M., \& Gallotta, A. (2016). Lateral flow assays. Essays in biochemistry, 60(1), 111-20.

[14] Wong, R. and Tse, H. (2009) Lateral Flow Immunoassay. Humana Press, Totowa, NJ. http://www.diagnostics1.com/MANUAL/LFIA_Book[1].pdf

[15] nanoComposix. "Sample and Absorbent Pad Selection for Lateral Flow Assays." NanoComposix, nanocomposix.com/pages/sample-and-absorbent-pad-selectionfor-lateral-flow-assays.

[16] Millipore. (2008). Rapid Lateral Flow Test Strips: Considerations for Product Development. Billerica: Merck Millipore.

[17] Sajid, Muhammad, et al. (2015) "Designs, Formats and Applications of Lateral Flow Assay: A Literature Review.” Journal of Saudi Chemical Society, vol. 19, no. 6, pp. 689-705., doi:10.1016/j.jscs.2014.09.001.

[18] Wikimedia Commons. https://commons.wikimedia.org/wiki/File:Nitrocellulose-2Dskeletal.png. Nitrocellulose-2D-skeletal. Accessed March 3, 2019.

[19] A Guide to Lateral Flow Immunoassay Development.” Innova Biosciences, fnkprddata.blob.core.windows.net/domestic/download/pdf/IBS_A_guide_to_later al_flow_immunoassays.pdf.

[20] Holstein, C. A., Chevalier, A., Bennett, S., Anderson, C. E., Keniston, K., Olsen, C., ... Yager, P. (2015). Immobilizing affinity proteins to nitrocellulose: A toolbox 2115 for paper-based assay developers. Analytical and Bioanalytical Chemistry, 408(5), 1335-1346. doi:10.1007/s00216-015-9052-0

[21] Li, X. J., \& Zhou, Y. (Eds.). (2013). Microfluidic devices for biomedical applications. Retrieved from https://ebookcentral.proquest.com

[22] Elveflow. (2017). Paper Microfluidic Devices : A Review 2017. www.elveflow.com/microfluidic-tutorials/microfluidic-reviews-andtutorials/paper-microfluidic-devices-a-review-2017/.

[23] Martinez, A. W., Phillips, S. T., Butte, M. J., \& Whitesides, G. M. (2007). Patterned paper as a platform for inexpensive, low-volume, portable bioassays. Angewandte Chemie (International ed. in English), 46(8), 1318-20.

[24] Hu, Jie, et al. "Advances in Paper-Based Point-of-Care Diagnostics." Biosensors and Bioelectronics, vol. 54, 2014, pp. 585-597., doi:10.1016/j.bios.2013.10.075. 
[25] Martinez, A. W., et al. (2008). "Three-Dimensional Microfluidic Devices Fabricated in Layered Paper and Tape." Proceedings of the National Academy of Sciences, vol. 105, no. 50, , pp. 19606-19611., doi:10.1073/pnas.0810903105.

[26] Carrilho, E., Martinez, A. W., \& Whitesides, G. M. (2009). Understanding Wax Printing: A Simple Micropatterning Process for Paper-Based Microfluidics. Analytical Chemistry, 81(16), 7091-7095. doi:10.1021/ac901071p

[27] Lu, Y., Shi, W., Qin, J., \& Lin, B. (2010). Fabrication and Characterization of PaperBased Microfluidics Prepared in Nitrocellulose Membrane by Wax Printing. Analytical Chemistry, 82(1), 329-335. doi:10.1021/ac9020193

[28] Xia, Yanyan, et al. (2016) Fabrication Techniques for Microfluidic Paper-Based Analytical Devices and Their Applications for Biological Testing: A Review. Biosensors and Bioelectronics, vol. 77, pp. 774-789., doi:10.1016/j.bios.2015.10.032.

[29] Wang, Shoumei, et al. (2012). Paper-Based Chemiluminescence ELISA: Lab-onPaper Based on Chitosan Modified Paper Device and Wax-Screen-Printing. Biosensors and Bioelectronics, vol. 31, no. 1, pp. 212-218., doi:10.1016/j.bios.2011.10.019.

[30] Cheng, C., Martinez, A., Gong, J., Mace, C., Phillips, S., Carrilho, E., . . . Whitesides, G. (2010). Paper-Based ELISA. Angewandte Chemie International Edition, 49(28), 4771-4774. doi:10.1002/anie.201001005

[31] Hsu, C., Huang, H., Chen, W., Nishie, W., Ujiie, H., Natsuga, K., . . Cheng, C. (2014). Paper-Based ELISA for the Detection of Autoimmune Antibodies in Body Fluid - The Case of Bullous Pemphigoid. Analytical Chemistry, 86(9), 4605-4610. doi:10.1021/ac500835k

[32] Verma, M. S., Tsaloglou, M., Sisley, T., Christodouleas, D., Chen, A., Milette, J., \& Whitesides, G. M. (2018). Sliding-strip microfluidic device enables ELISA on paper. Biosensors and Bioelectronics, 99, 77-84. doi:10.1016/j.bios.2017.07.034

[33] Fridley, Gina E., et al. (2014). "Highly Sensitive Immunoassay Based on Controlled Rehydration of Patterned Reagents in a 2-Dimensional Paper Network." Analytical Chemistry, vol. 86, no. 13, pp. 6447-6453., doi:10.1021/ac500872j.

[34] Fernandes, S. C., Wilson, D. J., \& Mace, C. R. (2017). Fabrication of Threedimensional Paper-based Microfluidic Devices for Immunoassays. Journal of visualized experiments : JoVE, (121), 55287. doi:10.3791/55287

[35] Stevens, Dean Y., et al. (2008). Enabling a Microfluidic Immunoassay for the Developing World by Integration of on-Card Dry Reagent Storage. Lab on a Chip, vol. 8, no. 12, p. 2038., doi:10.1039/b811158h. 
[36] Washburn EW. (1921). The dynamics of capillary flow. Phys Rev. 17(3):273-283.

[37] Fu E, Ramsey S, Kauffman P, Lutz B, Yager P. Transport in two-dimensional paper networks. Microfluid Nanofluidics. 2011;10(1):29-35.

[38] Masoodi, Reza, and Krishna M. Pillai. (2010). Darcy's Law-Based Model for Wicking in Paper-like Swelling Porous Media. AIChE Journal, doi:10.1002/aic.12163.

[39] Schoelkopf, Joachim, et al. (2012). "Practical Observation of Deviation from LucasWashburn Scaling in Porous Media." Colloids and Surfaces A: Physicochemical and Engineering Aspects, vol. 206, no. 1-3, pp. 445-454., doi:10.1016/s09277757(02)00066-3.

[40] "Hemorrhagic Stroke.” Stroke.org, www.stroke.org/understand-stroke/what-isstroke/hemorrhagic-stroke/.

[41] Radiological Society of North America. "Stroke." RadiologyInfo.org, www.radiologyinfo.org/en/info.cfm?pg=stroke.

[42] Harvard Health Publishing. "Hemorrhagic Stroke." Harvard Health Blog, Harvard Health Publishing, www.health.harvard.edu/a_to_z/hemorrhagic-stroke-a-to-z.

[43] "Stroke." Mayo Clinic, Mayo Foundation for Medical Education and Research, 26 Oct. 2018, www.mayoclinic.org/diseases-conditions/stroke/diagnosistreatment/drc-20350119.

[44] The Internet Stroke Center. An Independent Web Resource for Information about Stroke Care and Research., www.strokecenter.org/patients/strokediagnosis/imaging-tests/ct-scan/.

[45] Musuka, T. D., Wilton, S. B., Traboulsi, M., \& Hill, M. D. (2015). Diagnosis and management of acute ischemic stroke: speed is critical. CMAJ : Canadian Medical Association journal = journal de l'Association medicale canadienne, 187(12), 887-93.

[46] Jickling, G. C., \& Sharp, F. R. (2015). Biomarker Panels in Ischemic Stroke. Stroke, 46(3), 915-920. doi:10.1161/strokeaha.114.005604

[47] Whiteley, W., Tseng, M., \& Sandercock, P. (2008). Blood Biomarkers in the Diagnosis of Ischemic Stroke. Stroke, 39(10), 2902-2909. doi:10.1161/strokeaha.107.511261 
[48] Beer, C., Blacker, D., Bynevelt, M., Hankey, G. J., \& Puddey, I. B. (2010). Systemic markers of inflammation are independently associated with S100B concentration: Results of an observational study in subjects with acute ischaemic stroke. Journal of Neuroinflammation, 7(1), 71. doi:10.1186/1742-2094-7-71

[49] Rothermundt, M., Peters, M., Prehn, J. H., \& Arolt, V. (2003). S100B in brain damage and neurodegeneration. Microscopy Research and Technique, 60(6), 614632. doi:10.1002/jemt. 10303

[50] Ramachandran, S., Fu, E., Lutz, B., \& Yager, P. (2014). Long-term dry storage of an enzyme-based reagent system for ELISA in point-of-care devices. The Analyst, 139(6), 1456-62.

[51] Peeling, R. W., Holmes, K. K., Mabey, D., \& Ronald, A. (2006). Rapid tests for sexually transmitted infections (STIs): The way forward. Sexually Transmitted Infections, 82(Suppl_5), V1-V6. doi:10.1136/sti.2006.024265

[52] Clayton, K. N. (2012). Comparing Anti-VEGF Antibodies and Aptamers on Paper Microfluidic-Based Platforms. doi:10.15368/theses.2012.83

[53] Ward, J. G. (2012). Nitrocellulose Paper Based Microfluidic Platform Development and Surface Functionalization with Anti-IgE Aptamers. doi:10.15368/theses.2012.68

[54] Tageson, M. E. (2013). Functional 3-D Cellulose \& Nitrocellulose Paper-Based, Mulitplex Diagnostic Platforms Without Coupling Agents. doi:10.15368/theses.2013.208

[55] Young, M. (2016). A 3-D Multiplex Paper-microfluidic Platform. Retrieved from https://digitalcommons.calpoly.edu/theses/1680.

[56] Holler, A. (2016). Functional 3-D Cellulose and Nitrocellulose Paper-based, Microfluidic Device Utilizing ELISA Technology for the Detection/Distinction Between Hemorrhagic and Ischemic Strokes. Retrieved from https://digitalcommons.calpoly.edu/theses/1707.

[57] Daugherty, B. T. (2015). Improved Detection of Gold Nanoparticle Labels for Paper-based Analytics. doi:10.15368/theses.2015.33

[58] Van Huynh, E. (2016). Development of a 3-D Microfluidic Paper-Based Analytical Device for Elisa Reagents and a Smartphone Image Analysis Diagnostic Algorithm.

[59] Rasband, W. (2004). RGB Measure. Retrieved from https://imagej.nih.gov/ij/plugins/rgb-measure.html 
[60] Armbruster, D. A., \& Pry, T. (2008). Limit of blank, limit of detection and limit of quantitation. The Clinical biochemist. Reviews, 29 Suppl 1(Suppl 1), S49-S52.

[61] Cardillo G. (2012) Four parameters logistic regression - There and back again https://it.mathworks.com/matlabcentral/fileexchange/38122

[61] Drummond, J. E. (n.d.). Four Parameter Logistic Regression. Retrieved from https://www.myassays.com/four-parameter-logistic-regression.html

[62] Shrivastava, A., \& Gupta, V. (2011). Methods for the determination of limit of detection and limit of quantitation of the analytical methods. Chronicles of Young Scientists, 2(1), 21. doi:10.4103/2229-5186.79345

[63] Human S100B / S100 Beta Protein (Recombinant 6His,N-terminus) (Full Length) LS-G26341. (n.d.). Retrieved from https://www.lsbio.com/proteins/human-s100bs100-beta-protein-recombinant-6hisn-terminus-full-length-lsg26341/26341?trid=247

[64] CT Scan of the Head: About This Test. (n.d.). Retrieved from https://myhealth.alberta.ca/Health/aftercareinformation/pages/conditions.aspx?hw $\mathrm{id}=\mathrm{ug} 6566$

[65] S100B ELISA Kit (ab234573). (2019, May 19). Retrieved from https://www.abcam.com/s100b-elisa-kit-ab234573.html

[66] S100B ELISA Kit (Human) : 96 Wells (OKCA00128). (n.d.). Retrieved from https://www.avivasysbio.com/s100b-elisa-kit-human-96-wells-okca00128.html

[67] Dassan, P., Keir, G., \& Brown, M. M. (2009). Criteria for a Clinically Informative Serum Biomarker in Acute Ischaemic Stroke: A Review of S100B. Cerebrovascular Diseases, 27(3), 295-302. doi:10.1159/000199468

[68] Selcuk, O., Yayla, V., Cabalar, M., Guzel, V., Uysal, S., \& Gedikbasi, A. (2014). The Relationship of Serum S100B Levels with Infarction Size and Clinical Outcome in Acute Ischemic Stroke Patients. Noro Psikiyatri Arsivi, 51(4), 395400. doi:10.5152/npa.2014.7213

[69] Lu, Y., Wang, R., Huang, H., Qin, H., Liu, C., Xiang, Y., .. . Wei, Y. (2018). Association of S100B polymorphisms and serum S100B with risk of ischemic stroke in a Chinese population. Scientific Reports, 8(1). doi:10.1038/s41598-01819156-w

[70] Jones, Kevin. (2008). FUSION 5: A New Platform For Lateral Flow Immunoassay Tests. 10.1007/978-1-59745-240-3_7. 


\section{APPENDICES}

\section{A. AutoCAD Dimensioned Drawings}

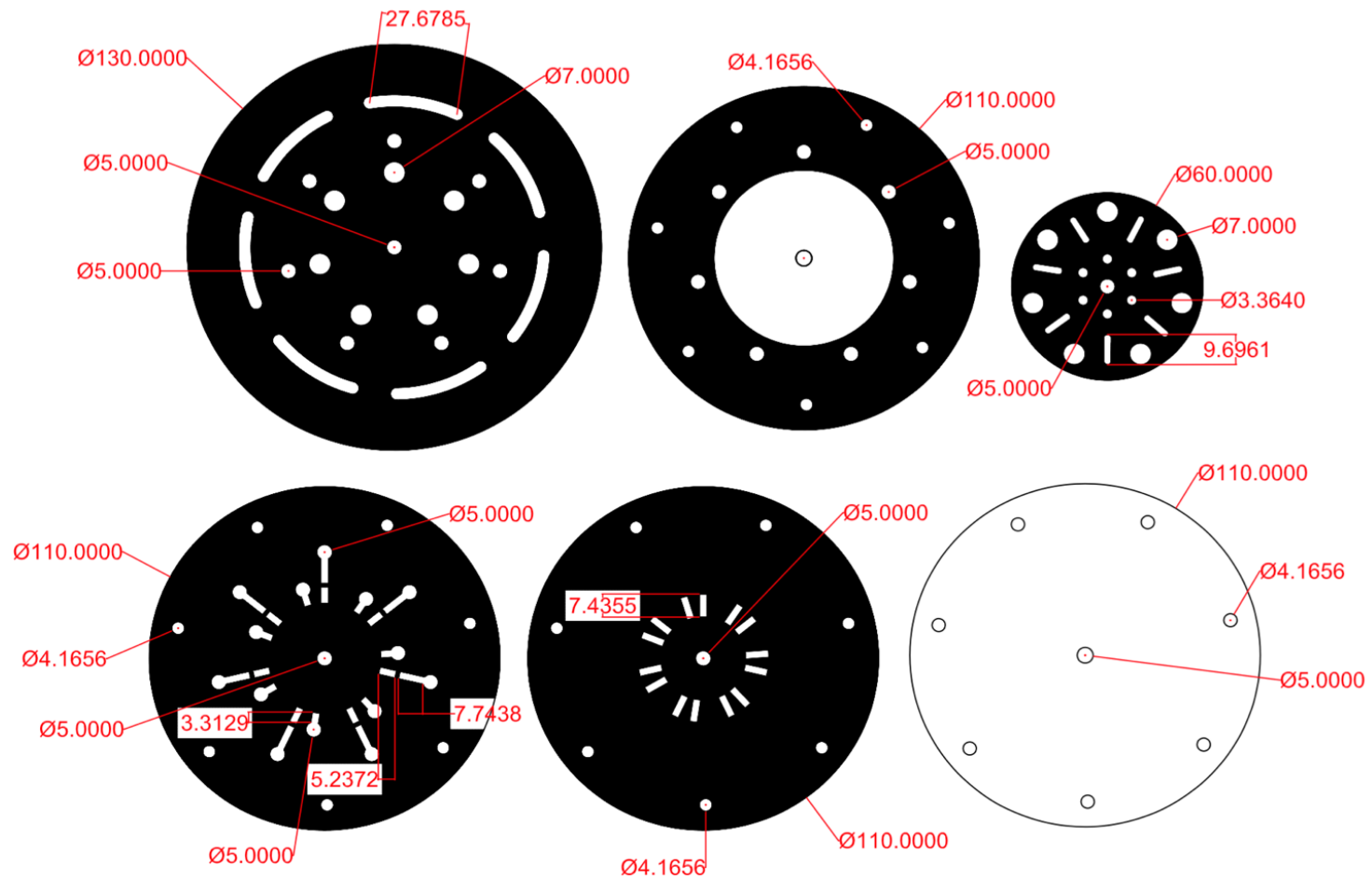

Figure 50. Dimensioned drawing of Holler (2016) device design

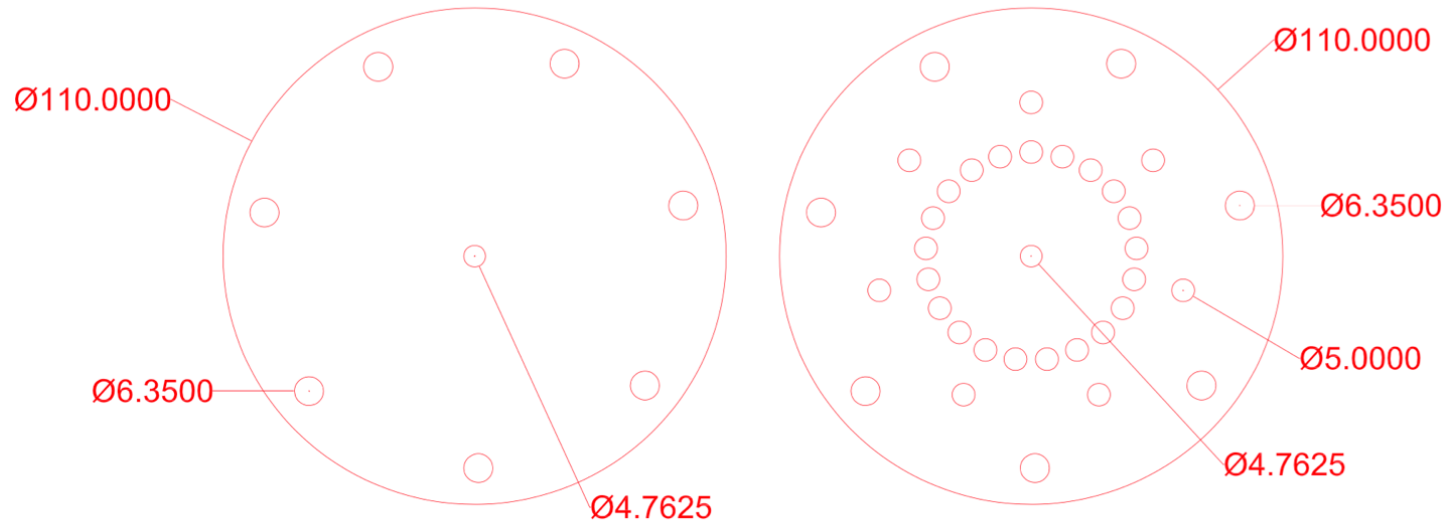

Figure 51. Dimensioned drawing of Holler (2016) housing design 

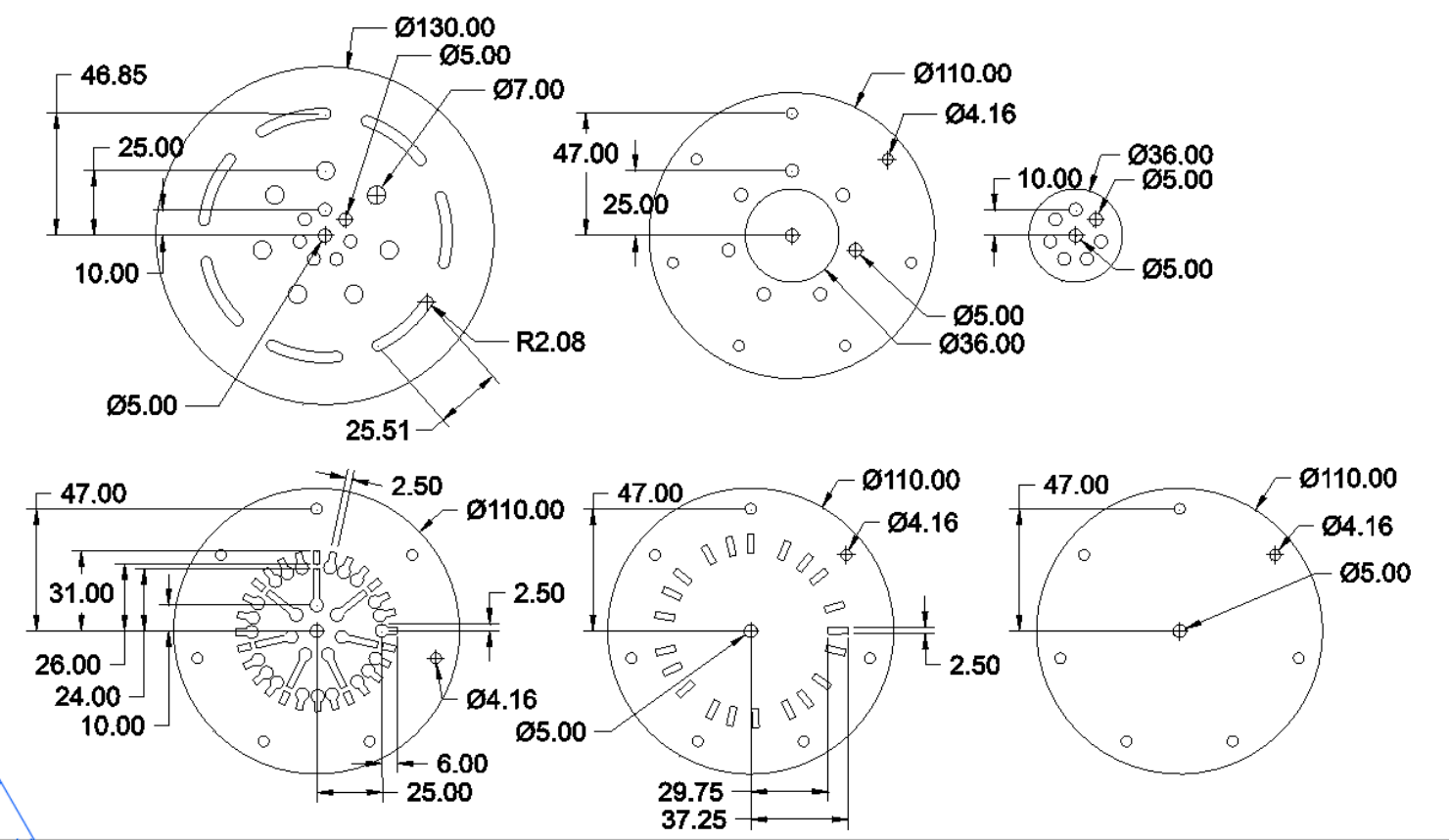

Figure 52. Dimensioned initial rotational outward flow design

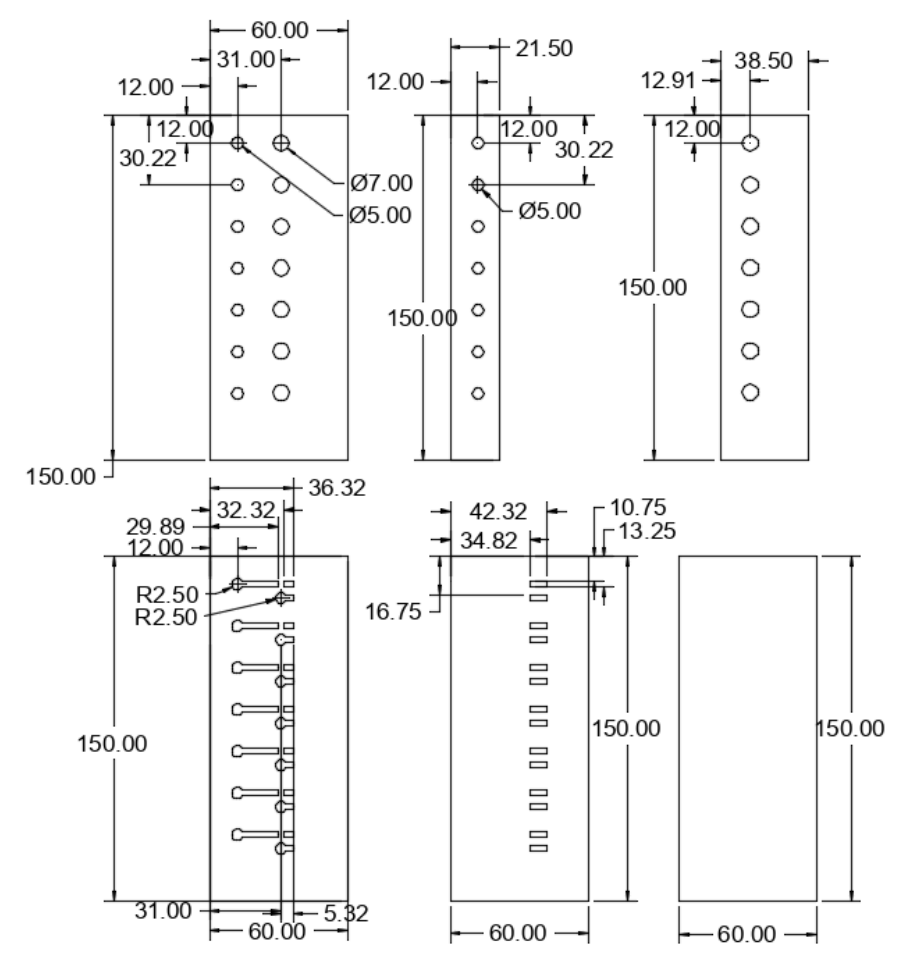

Figure 53. Dimensioned lateral motion pull strip design 


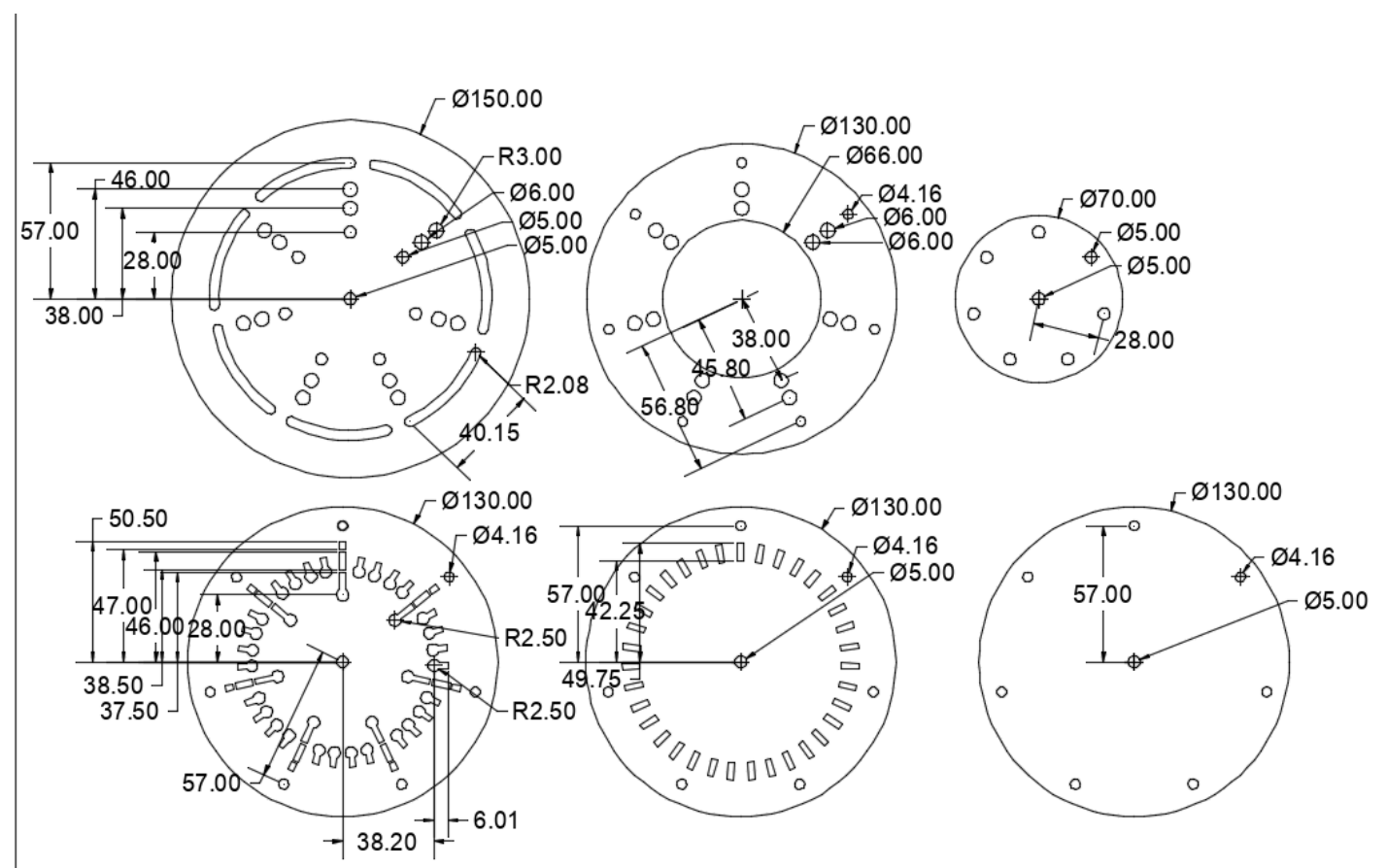

Figure 54. Dimensioned outward flow design with four channels and a control pad

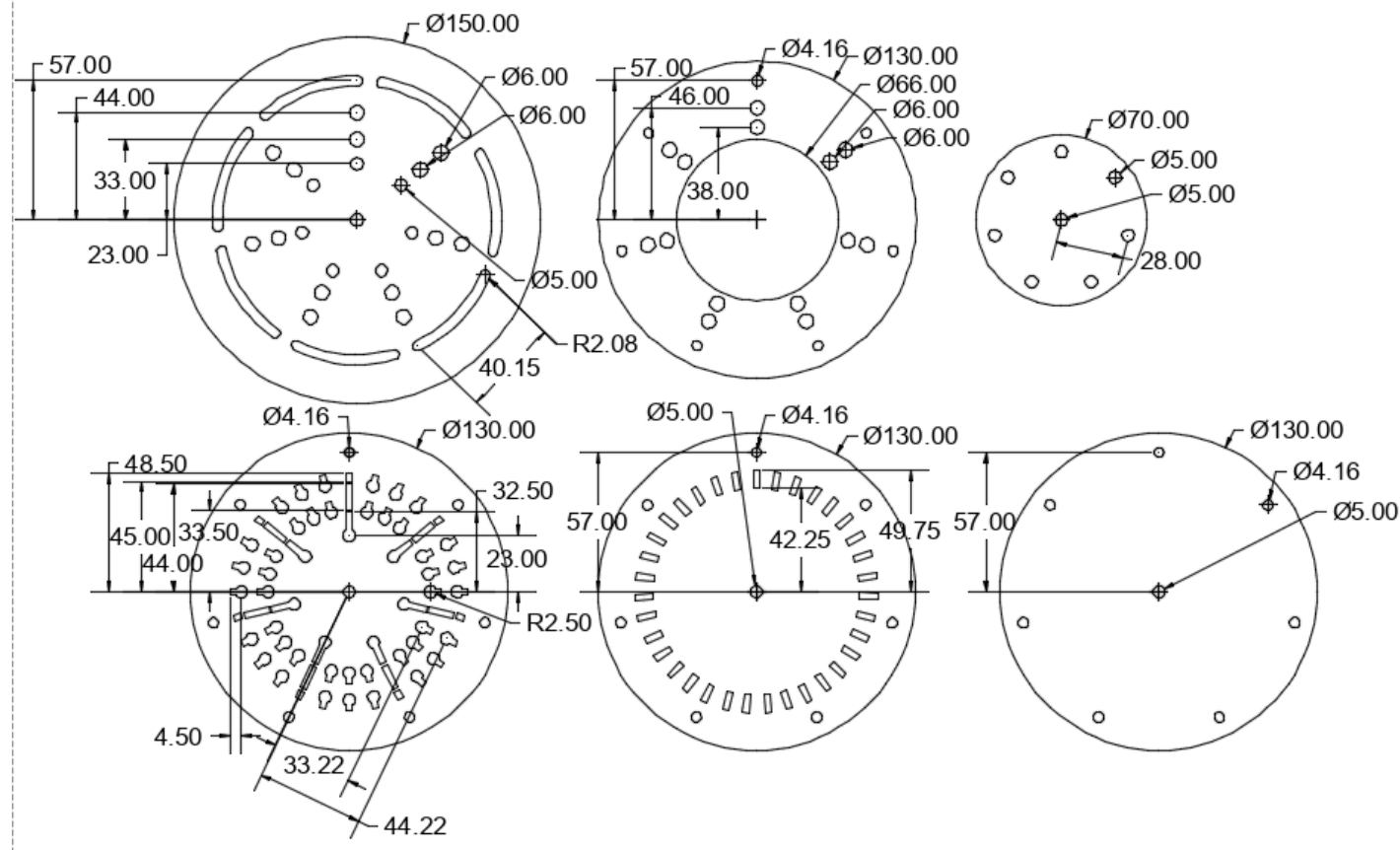

Figure 55. Dimensioned outward flow design with three detection pad channels and three control pad channels 

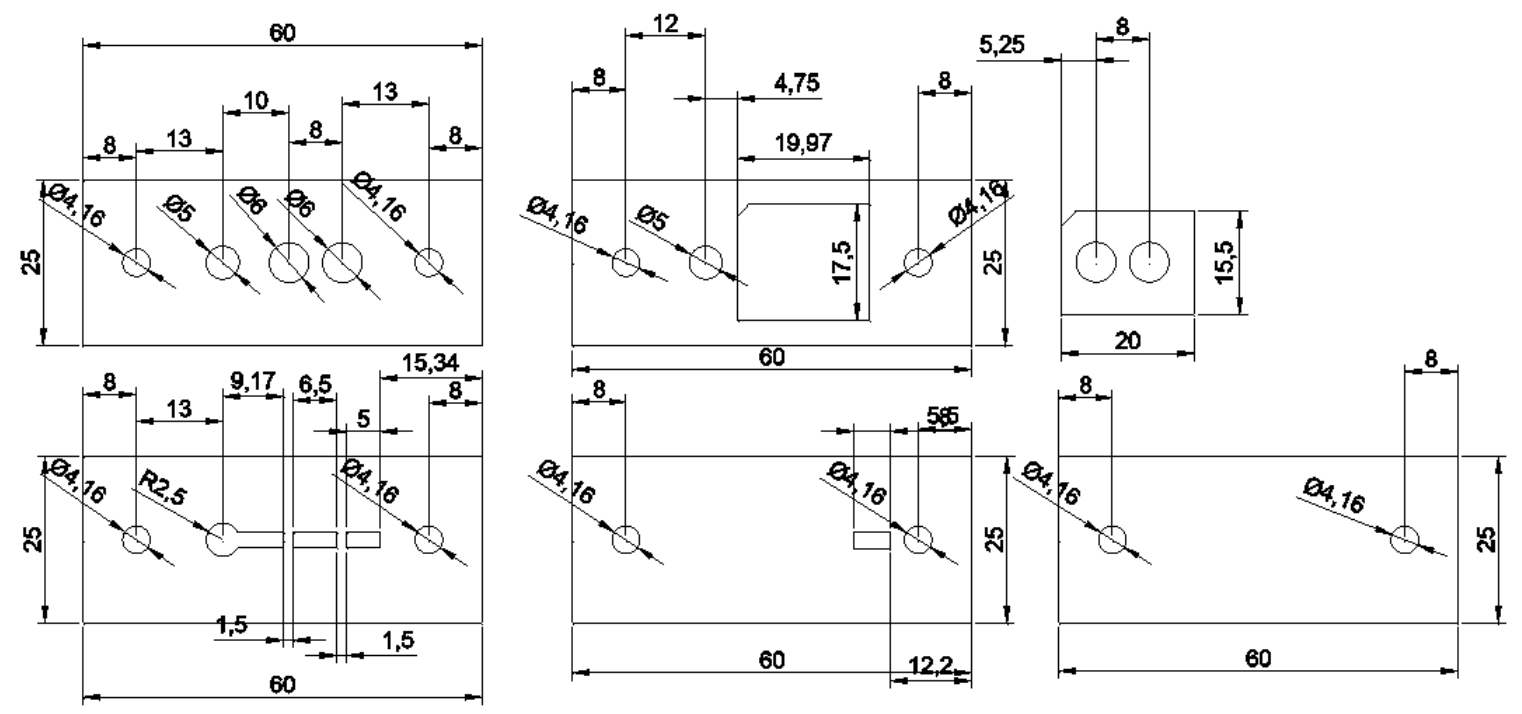

Figure 56. Dimensions of final design for one sample fluid flow validation test
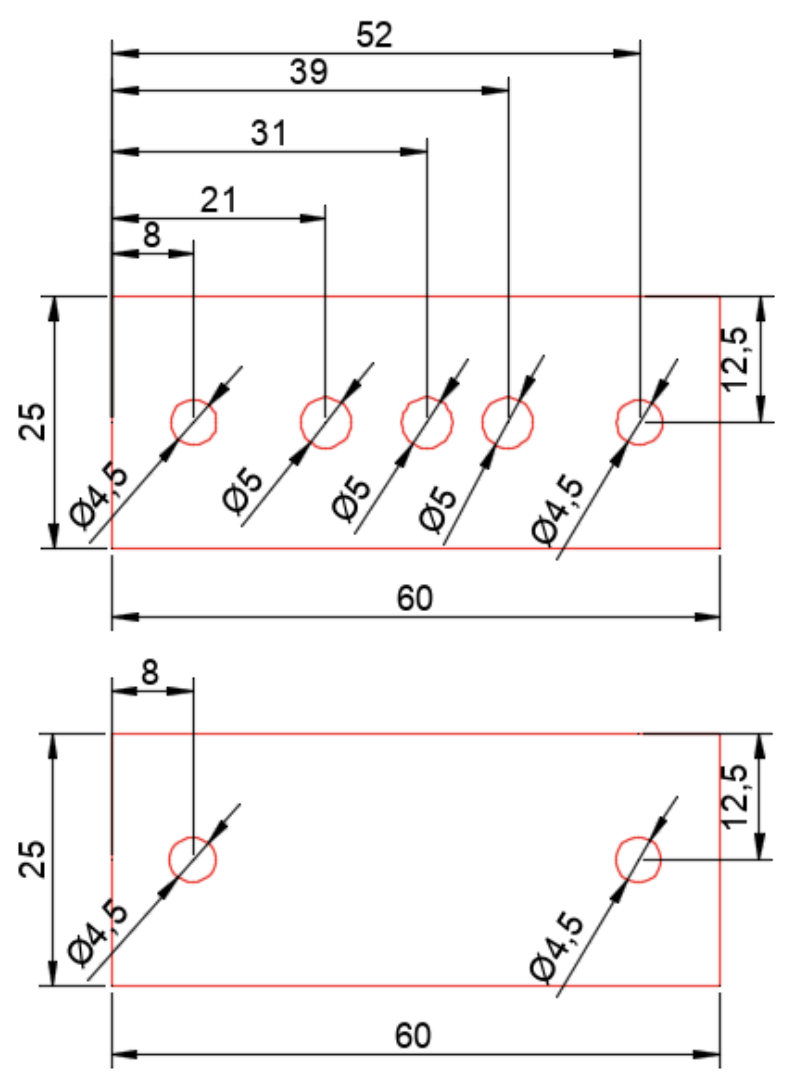

Figure 57. Dimensions of single sample device housing 


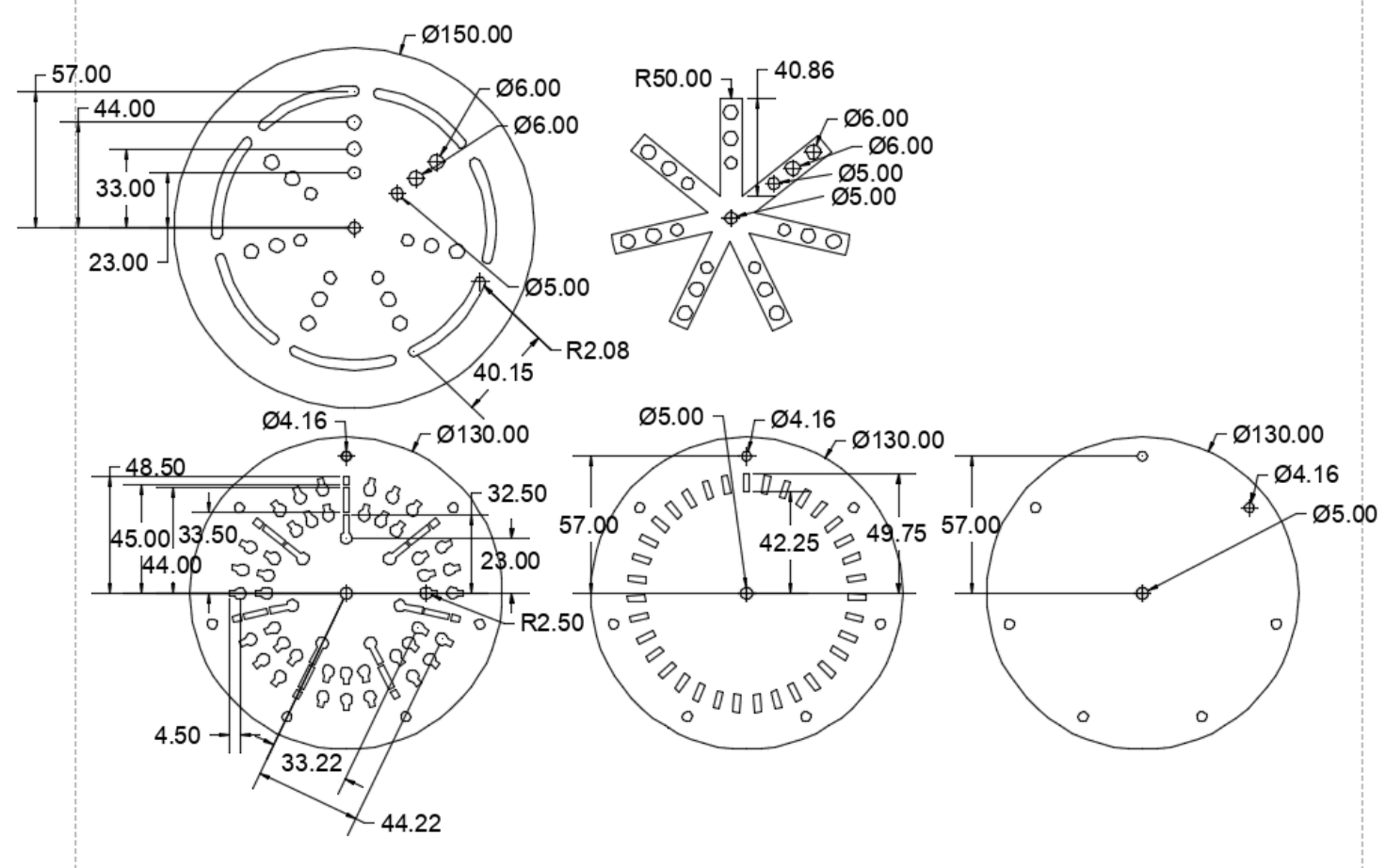

Figure 58. Dimensioned nitrocellulose star design

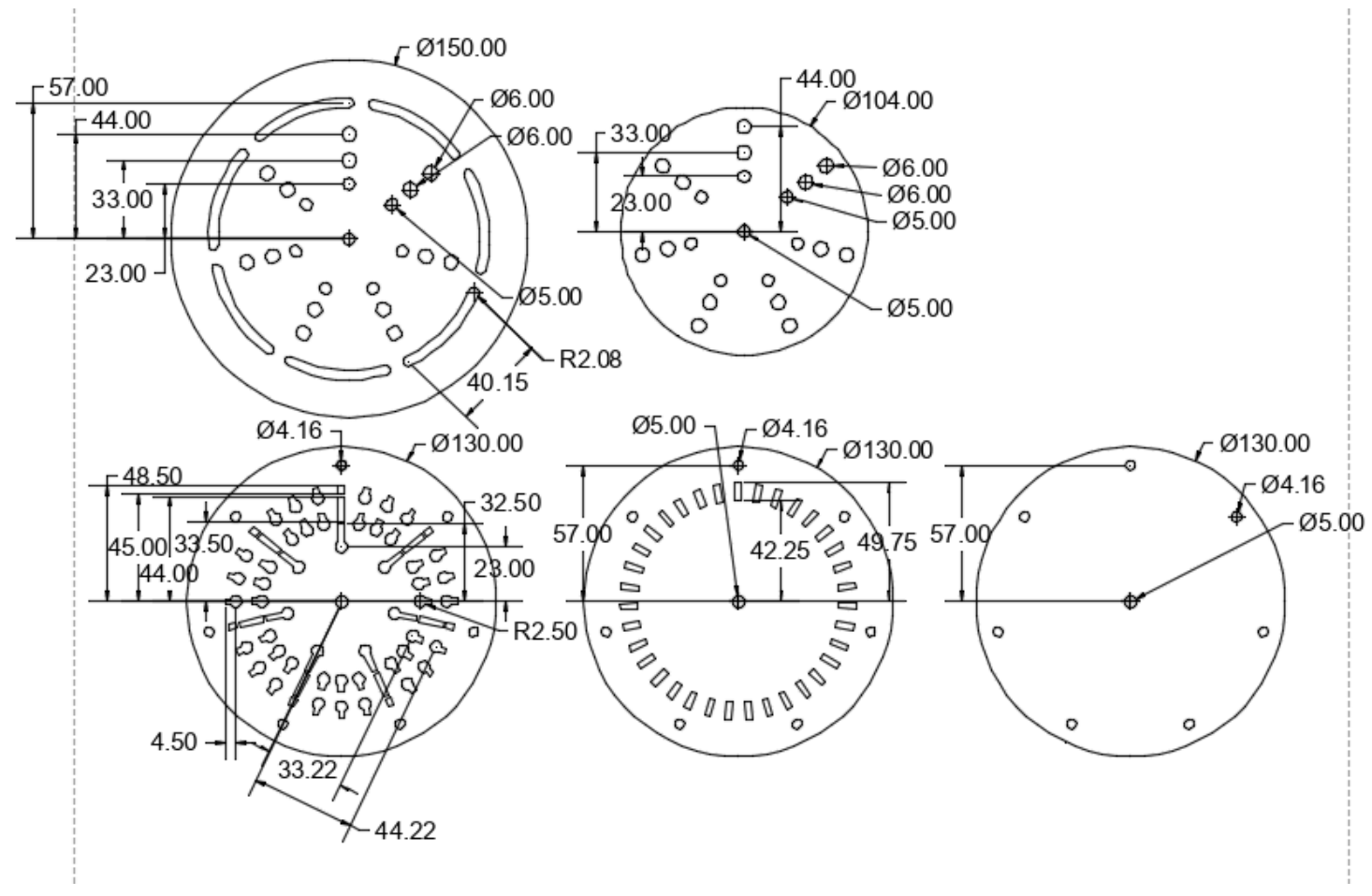

Figure 59. Dimensioned final design for the paper-based $\mu$ PAD 

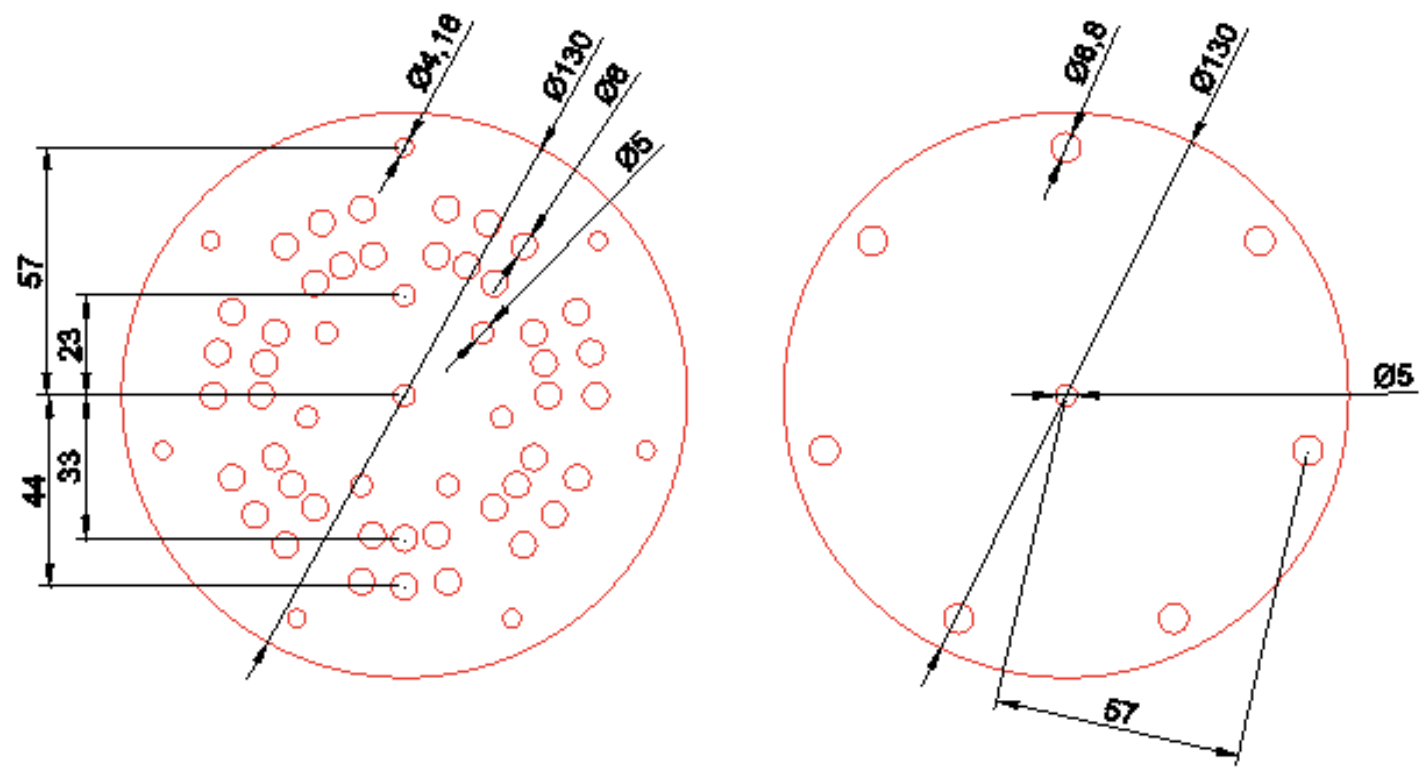

Figure 60. Dimensioned final design for the paper-based $\mu$ PAD housing

\section{B. Enzyme Assay Development}

Table XIV. High Concentration Dose Response - Trial 1

\begin{tabular}{|c|c|c|c|}
\hline \multicolumn{4}{|c|}{ High Concentration Set - Trial 1} \\
\hline $\begin{array}{c}\text { Concentration } \\
(\mathrm{ng} / \mathrm{ml})\end{array}$ & $\begin{array}{l}\text { Average Color } \\
\text { Intensity }\end{array}$ & $\begin{array}{c}\text { Concentration } \\
(\mathrm{ng} / \mathrm{ml})\end{array}$ & $\begin{array}{l}\text { Signal to } \\
\text { Noise }\end{array}$ \\
\hline 100000 & 88.232 & 100000 & 2.438025958 \\
\hline 10000 & 103.393 & 10000 & 2.685123513 \\
\hline 1000 & 93.70566667 & 1000 & 2.527237069 \\
\hline 100 & 90.57833333 & 100 & 2.476267052 \\
\hline 10 & 82.048 & 10 & 2.337237666 \\
\hline 1 & 100.0033333 & 1 & $2.629877926^{*}$ \\
\hline 0.1 & 35.67733333 & 0.1 & 1.581477598 \\
\hline
\end{tabular}

* Designates data which was removed for the corrected dose response data set 
Table XV. High Concentration Dose Response - Trial 2

\begin{tabular}{|c|c|c|c|}
\hline \multicolumn{4}{|c|}{ High Concentration Set - Trial 2} \\
\hline $\begin{array}{c}\text { Concentration } \\
(\mathrm{ng} / \mathrm{ml})\end{array}$ & $\begin{array}{l}\text { Average Color } \\
\text { Intensity }\end{array}$ & $\begin{array}{c}\text { Concentration } \\
(\mathrm{ng} / \mathrm{ml})\end{array}$ & $\begin{array}{l}\text { Signal to } \\
\text { Noise }\end{array}$ \\
\hline 100000 & 33.40633333 & 100000 & 2.434179081 \\
\hline 10000 & 18.27966667 & 10000 & 1.784770818 \\
\hline 1000 & 27.551 & 1000 & 2.1828017 \\
\hline 100 & 17.21333333 & 100 & 1.738991686 \\
\hline 10 & 20.02833333 & 10 & 1.859843444 \\
\hline 1 & 13.01666667 & 1 & 1.558823109 \\
\hline 0.1 & 10.38566667 & 0.1 & 1.445870719 \\
\hline
\end{tabular}

Table XVI. High Concentration Dose Response - Trial 3

\begin{tabular}{|r|r|}
\hline \multicolumn{1}{|c|}{$\begin{array}{c}\text { High Concentration } \\
\text { (ng/ml) }\end{array}$} & $\begin{array}{c}\text { Average Color } \\
\text { Intensity }\end{array}$ \\
\hline 100000 & 34.835 \\
\hline 10000 & 37.25633333 \\
\hline 1000 & 38.45033333 \\
\hline 100 & 39.491 \\
\hline 10 & 36.636 \\
\hline 1 & 23.98033333 \\
\hline 0.1 & 15.66166667 \\
\hline
\end{tabular}

\begin{tabular}{|r|r|}
$\begin{array}{c}\text { Concentration } \\
(\mathbf{n g} / \mathbf{m l})\end{array}$ & $\begin{array}{c}\text { Signal to } \\
\text { Noise }\end{array}$ \\
\hline 100000 & 2.325112534 \\
\hline 10000 & 2.417219299 \\
\hline 1000 & 2.462638686 \\
\hline 100 & 2.502225322 \\
\hline 10 & 2.393622012 \\
\hline 1 & 1.9122044 \\
\hline 0.1 & 1.595764915 \\
\hline
\end{tabular}

Table XVII. Low Concentration Dose Response - Trial 1

\begin{tabular}{|c|c|c|c|}
\hline \multicolumn{4}{|c|}{ Low Concentration Set - Trial 1} \\
\hline $\begin{array}{c}\text { Concentration } \\
(\mathrm{ng} / \mathrm{ml})\end{array}$ & $\begin{array}{l}\text { Average Color } \\
\text { Intensity }\end{array}$ & $\begin{array}{c}\text { Concentration } \\
(\mathrm{ng} / \mathbf{m l})\end{array}$ & $\begin{array}{l}\text { Signal to } \\
\text { Noise }\end{array}$ \\
\hline 100 & 26.953 & 100 & 1.413347507 \\
\hline 10 & -- & 10 & -- \\
\hline 1 & 19.87266667 & 1 & 1.253206071 \\
\hline 0.1 & 21.472 & 0.1 & 1.289379443 \\
\hline 0.01 & 18.88266667 & 0.01 & 1.230814466 \\
\hline 0.001 & 27.943 & 0.001 & $1.435739111^{*}$ \\
\hline 0.0001 & 20.563 & 0.0001 & 1.26881988 \\
\hline
\end{tabular}

* Designates data which was removed for the corrected dose response data set 
Table XVIII. Low Concentration Dose Response - Trial 2

\begin{tabular}{|c|c|c|c|}
\hline \multicolumn{4}{|c|}{ Low Concentration Set - Trial 2} \\
\hline $\begin{array}{c}\text { Concentration } \\
(\mathrm{ng} / \mathrm{ml})\end{array}$ & $\begin{array}{c}\text { Average Color } \\
\text { Intensity }\end{array}$ & $\begin{array}{c}\text { Concentration } \\
(\mathrm{ng} / \mathrm{ml})\end{array}$ & $\begin{array}{l}\text { Signal to } \\
\text { Noise }\end{array}$ \\
\hline 100 & 26.061 & 100 & 1.991352311 \\
\hline 10 & 29.295 & 10 & 2.114372662 \\
\hline 1 & 22.433 & 1 & 1.853344323 \\
\hline 0.1 & 24.78933333 & 0.1 & 1.942978508 \\
\hline 0.01 & 23.72433333 & 0.01 & 1.90246624 \\
\hline 0.001 & 11.37333333 & 0.001 & 1.432638052 \\
\hline 0.0001 & 3.274666667 & 0.0001 & 1.124567299 \\
\hline
\end{tabular}

Table XIX. Low Concentration Dose Response - Trial 3

\begin{tabular}{|c|c|c|c|}
\hline \multicolumn{4}{|c|}{ Low Concentration Set - Trial 3} \\
\hline $\begin{array}{c}\text { Concentration } \\
(\mathrm{ng} / \mathrm{ml})\end{array}$ & $\begin{array}{c}\text { Average Color } \\
\text { Intensity }\end{array}$ & $\begin{array}{c}\text { Concentration } \\
(\mathrm{ng} / \mathrm{ml})\end{array}$ & $\begin{array}{l}\text { Signal to } \\
\text { Noise }\end{array}$ \\
\hline 100 & 15.618 & 100 & 1.594103848 \\
\hline 10 & 21.97033333 & 10 & 1.835744627 \\
\hline 1 & 14.93733333 & 1 & 1.568211501 \\
\hline 0.1 & 34.84766667 & 0.1 & $2.32559437^{*}$ \\
\hline 0.01 & 27.50166667 & 0.01 & $2.046154822 *$ \\
\hline 0.001 & 6.589333333 & 0.001 & 1.250656185 \\
\hline 0.0001 & 8.294 & 0.0001 & 1.315501173 \\
\hline
\end{tabular}

* Designates data which was removed for the corrected dose response data set

Table XX. Average of All Trials - Uncorrected

\begin{tabular}{|r|r|}
\hline \multicolumn{2}{|c|}{ All Trials Averaged } \\
\hline Concentration (ng/ml) & \multicolumn{1}{|c|}{ Signal to Noise } \\
\hline 100000 & 2.399105858 \\
\hline 10000 & 2.295704543 \\
\hline 1000 & 2.390892485 \\
\hline 100 & 1.952714621 \\
\hline 10 & 2.108164082 \\
\hline 1 & 1.795944555 \\
\hline 0.1 & 1.696844259 \\
\hline 0.01 & 1.726478509 \\
\hline 0.001 & 1.373011116 \\
\hline 0.0001 & 1.236296117 \\
\hline
\end{tabular}




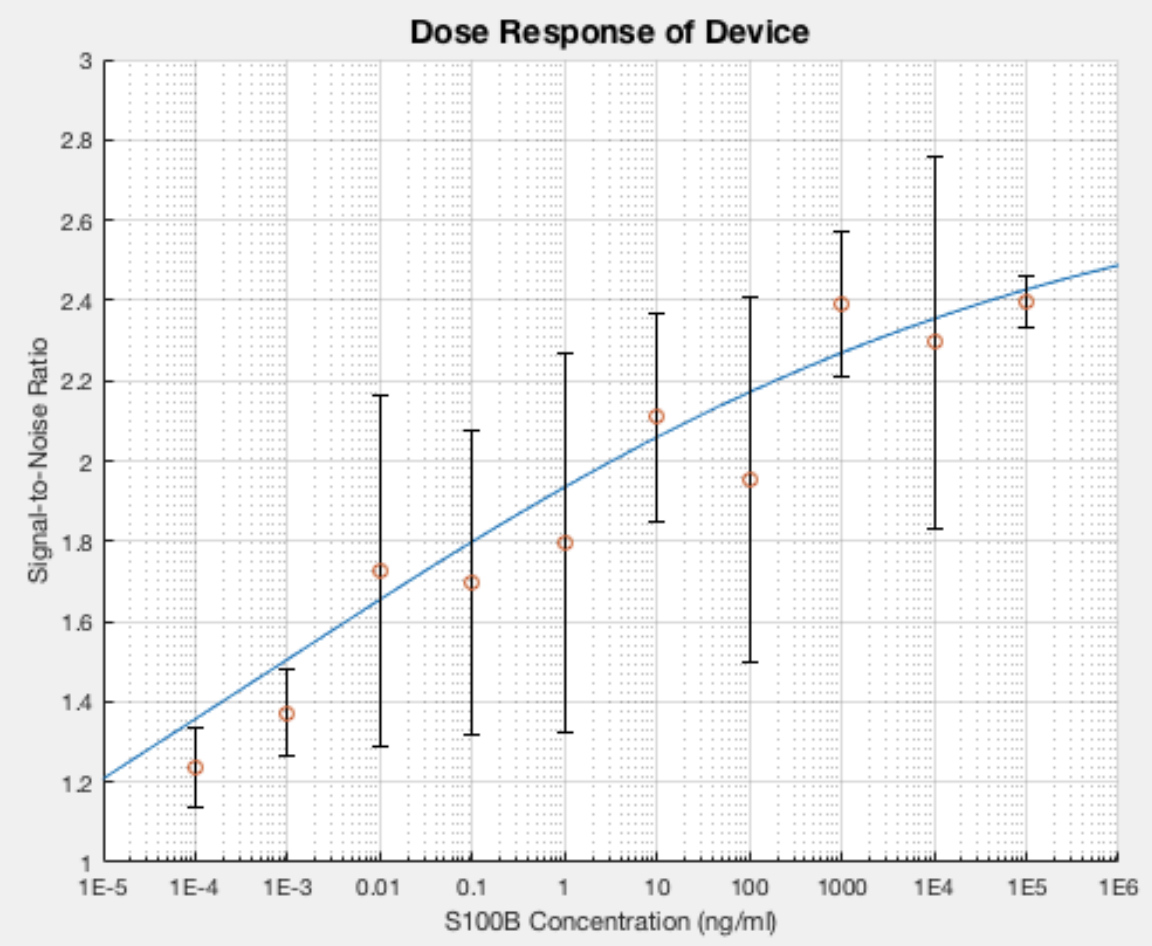

Figure 61. Uncorrected dose response graph

The equation for the 4PL fit was determined, shown below, with an $\mathrm{R}^{2}$ value of 0.9340 , indicating a good fit to the data.

$$
y=2.751+\frac{8.271 * 10^{-7}-2.751}{1+\left(\frac{x}{0.0005394}\right)^{0.1017}}
$$

Table XXI. Average of All Trials - Corrected

\begin{tabular}{|r|r|}
\hline \multicolumn{2}{|c|}{ Corrected Trials Averaged } \\
\hline Concentration (ng/ml) & Signal to Noise \\
\hline 100000 & 2.399105858 \\
\hline 10000 & 2.295704543 \\
\hline 1000 & 2.390892485 \\
\hline 100 & 1.952714621 \\
\hline 10 & 2.108164082 \\
\hline 1 & 1.62915788 \\
\hline 0.1 & 1.571094237 \\
\hline 0.01 & 1.566640353 \\
\hline 0.001 & 1.341647118 \\
\hline 0.0001 & 1.236296117 \\
\hline &
\end{tabular}




\begin{tabular}{|r|r|} 
Concentration (ng/ $\mathbf{m l})$ & Average Color Intensity \\
\hline 100000 & 52.15777778 \\
\hline 10000 & 52.97633333 \\
\hline 1000 & 53.23566667 \\
\hline 100 & 35.98577778 \\
\hline 10 & 37.99553333 \\
\hline 1 & 32.37388889 \\
\hline 0.1 & 23.80561111 \\
\hline 0.01 & 23.36955556 \\
\hline 0.001 & 15.30188889 \\
\hline 0.0001 & 10.71055556 \\
\hline
\end{tabular}

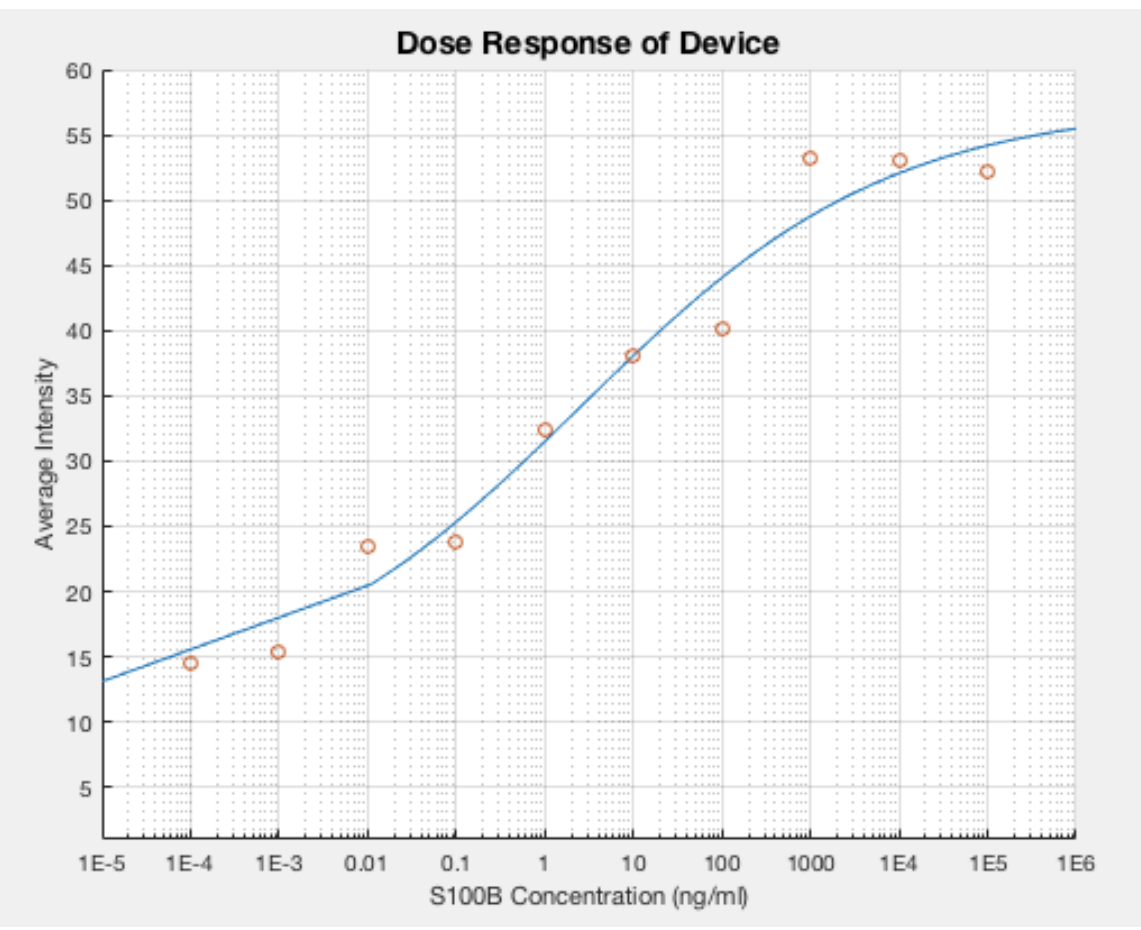

Figure 62. Dose response of device for average intensity

The equation for the 4PL fit was determined, shown below, with an $\mathrm{R}^{2}$ value of 0.9731 , indicating a good fit to the data.

$$
y=57.27+\frac{11.13-57.27}{1+\left(\frac{x}{2.573}\right)^{0.2493}}
$$

